\title{
Animal camouflage: \\ disentangling disruptive coloration from \\ background matching
}

\author{
by \\ Richard James Webster
}

A thesis submitted to the Faculty of Graduate and Postdoctoral Affairs in partial

fulfillment of the requirements for the degree

Doctor of Philosophy

in

Biology

Carleton University

Ottawa, Ontario

(C) 2013

Richard James Webster 


\section{Abstract}

Camouflage is ubiquitous in the natural world and provides adaptive benefits to both predators and their prey. In this study I test concepts of animal camouflage using the experimental paradigm of humans foraging for real and artificial moth targets on a computer screen and assessed camouflage efficacy by measuring detection rates. Chapter 1 outlines the questions and objectives of this doctoral thesis. In Chapter 2 I introduce the phenomenon of disruptive coloration, followed by a brief-review of the visual mechanisms contributing to visual search. Chapter 3 tested if non-random orientation behaviour of moths in the field could be explained by behaviourally-mediated camouflage. I showed that the preferred fieldorientations of moths were associated with lower detection rates in the lab, and that the relative orientation of the moth to the tree was the key driver. Chapter 4 tested the fundamental assumption that disruptive coloration functions by impairing shape perception. It was predicted that if edge-intersecting patches are disruptive, then altering the shape of a target would interact with edge coloration. Artificial moth-like targets did show an interaction between edge coloration and target shape, which explained detectability. These findings suggest that effectiveness of camouflage due to edge markings is dependent on target shape, which further supports the hypothesis that edge markings function as disruptive coloration. Chapter 5 took a similar approach to chapter 4 but tested if there was an interaction between edge coloration and target boundary visibility, which could explain detectability of moth-like targets. Results from Chapters 4 and 5 suggest that shape and boundary properties play a role in disruptive function of edge markings. Chapter 6 tested how this might occur. It is thought that edge-intersecting patches impair object recognition. It was predicted that moth-like targets with more edge-intersecting patches would be harder to recognise. Recognition was characterised by human foveal vision, monitored by eyetracking. Indeed, targets with a larger number of edge-intersecting patches were associated with being difficult to recognise, and reduced detectability even at the expense of background matching. 


\section{Acknowledgements}

Writing a thesis is like rowing a single scull. It looks peaceful and relaxing from the outside,but on the inside it is an endurance event for the mind accomplished with a lot of hard work: such challenges are made all the more enjoyable when others come along for the ride.

It has been a privilege to be mentored by and work alongside my co-supervisors, Profs Tom Sherratt and Jean-Guy Godin. I am grateful for their guidance, imparted knowledge and enthusiasm during my doctoral thesis. I am fortunate to have supervisors that are not only engaging but also have had my best interests at heart! I am particularly thankful for your bureaucracy battles in accepting me as an international student, supporting my attendance at CamoCon 2013, and encouraging my adventure to the '96 magnetic north pole with Row to the Pole. Also, thanks for the good times at Mike's Place.

My experience at Carleton University has been made all the more enjoyable with the very friendly and warm staff, faculty and peers of the Department of Biology. Special thanks must go to Jayne Yack, Gabriel Blouin-Demers, Kevin Abbott, Chris Hassall, Justin Carrol, Tom Hossie, Ian McLean, Allison Callahan, Tracy Kirkpatrick, Isabelle Letourneau, Zoe Panchen, Adam Smith, Stacey Lee Jenkins, Heather Auld, Lynn Scott, Kringen Henein and Jim des Rivieres. I would also like to thank Geoff White, Graham Warren, Rebecca Stiles, Jen Schellinck and Colin Henein for their friendship and moral support!

I would not have the enthusiasm for natural history and confidence to tackle doctoral studies without the encouragement and unwavering support of my parents. Thank you so much, and I look forward to our shared adventures ahead.

The final thanks must go to Francina Jackson for enduring my midnight note taking, 4:30 a.m. pre-work rowing, last minute editing requests and general detachment from life during parts of this process. I couldn't have done this without you by my side. 


\section{Preface}

\section{Co-authorship statement for Chapters 3 and 6}

Of the four data chapters included in this thesis, Chapter 3 is published in Philosophical Transactions of the Royal Society Biological Sciences and Chapter 6 is under review by Biology Letters. Dr. Tom Sherratt, Dr. Jean-Guy Godin and I collaboratively developed the research questions and experimental design for Chapter 3. Allison Callahan contributed by aiding with moth collection in the field. Dr. Sherratt and I designed the human foraging computer program. I performed data analysis for field data, data collection and analysis for human trials, and drafted the manuscript. Dr. Sherratt and Dr. Godin provided editorial suggestions to improve this manuscript.

I developed the project in Chapter 6 with collaboration from Dr. Sherratt and Dr. Godin. Dr. Chris Herdman provided access to eye-tracking equipment. I designed the computer program, oversaw the human trials, performed all analyses, and drafted the manuscript. Dr. Chris Hassall helped to refine the $\mathrm{G}_{\mathrm{z}} \mathrm{LMM}$ analysis. Dr. Sherratt, Dr. Godin and Dr. Hassall provided editorial suggestions that improved the manuscript.

For the purpose of this doctoral dissertation I often make use of the pronouns "I" and "my": I completely acknowledge the contributions of all co-authors and hope that they will allow me this discretion. 


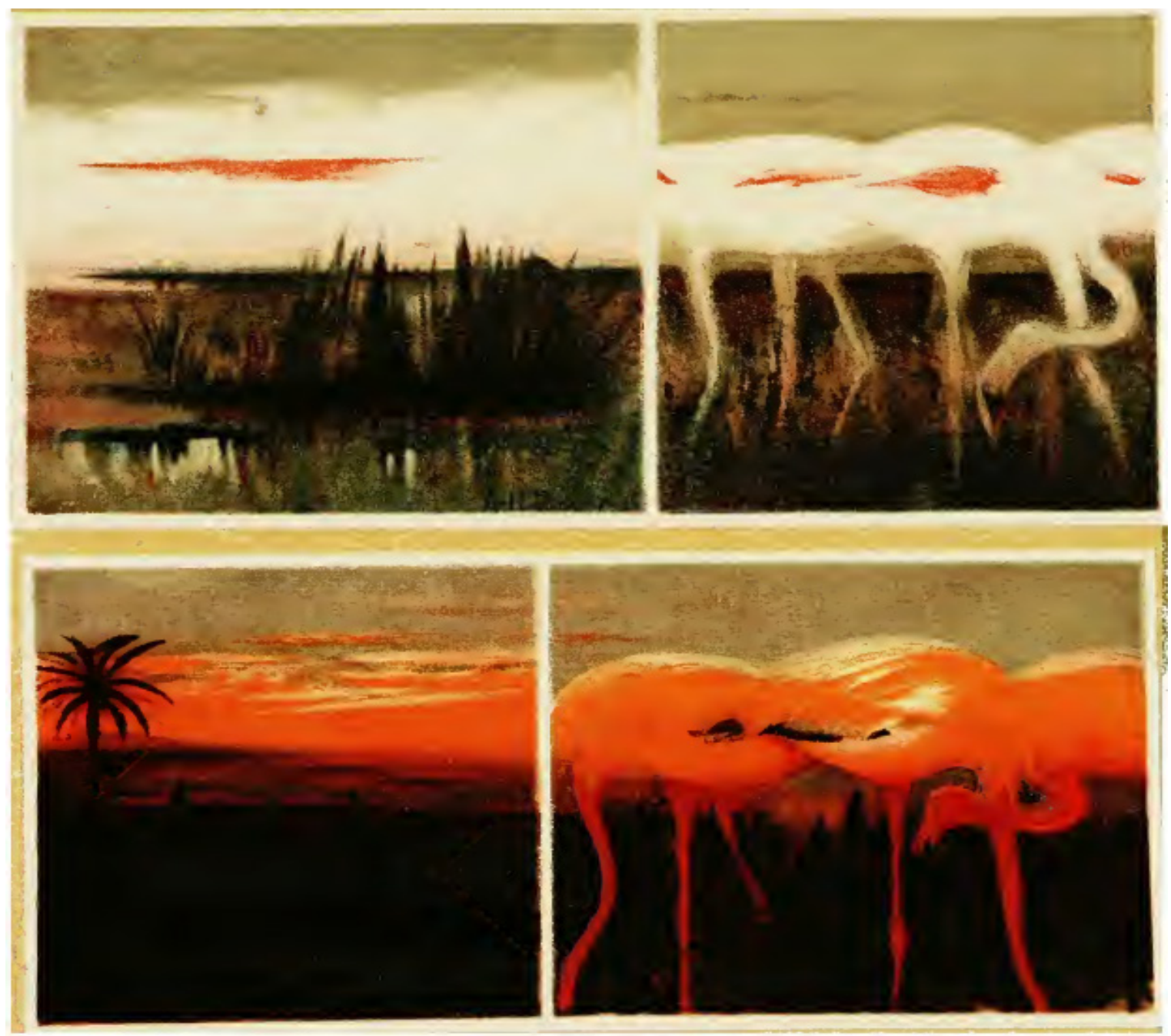

"These various sketches of Flamingoes ... show how wonderfully such birds match or reproduce the colors of morning or evening skies to the eyes of the inhabitants of the water... Commonly, the bird's upper outline 'melts' into the sky, leaving the rest of his contour, seen through the water, agitated and muddied by his feet, to be lost in the indeterminate color-mass of the flock." (Thayer, 1909)

Camouflaged flamingos are the most ludicrous of the 'just-so' stories of animal coloration and a symbolic warning (Gould, 1991); to claim an adaptive purpose of a camouflage coloration, scientists need to test its function. 


\section{Overview of the Thesis' Component Chapters}

\section{Chapter 1:}

General Introduction

Chapter 2:

A brief review of disruptive coloration from a ultimate and proximate context

\section{Chapter 3:}

Behaviourally mediated crypsis in two nocturnal moths with contrasting appearance

\section{Chapter 4:}

Disruptive coloration and the role of shape perception in animal camouflage

\section{Chapter 5:}

Disruptive coloration and boundary visibility: Testing functional predictions of disruptive coloration

\section{Chapter 6:}

Disruptive camouflage impairs object recognition

\section{Chapter 7:}

General Discussion 


\section{Table of Contents}

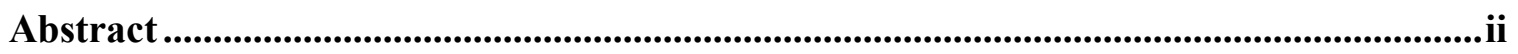

Acknowledgements....................................................................iii

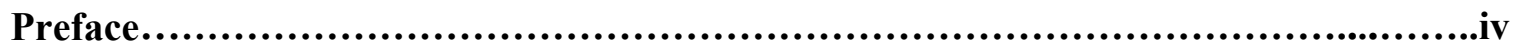

Overview of the Thesis' Component Chapters .............................................................................vi

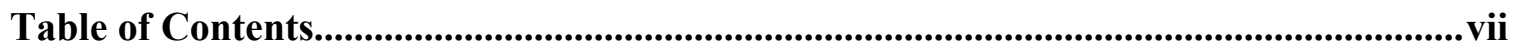

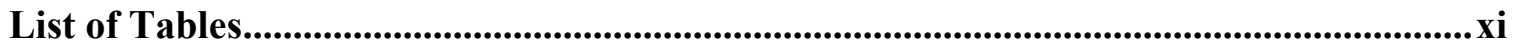

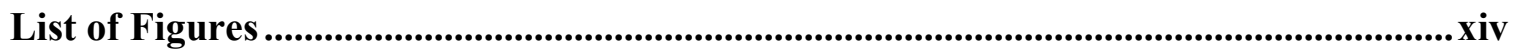

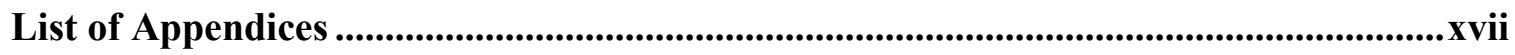

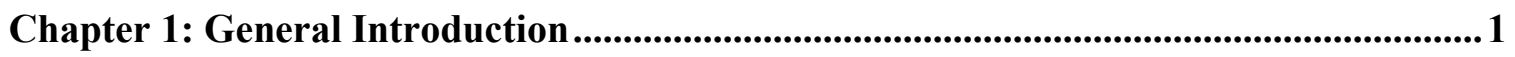

1.1 Animal camouflage and a century-old unanswered question ................................... 1

1.2 Categorizing animal colorations through their function ....................................... 7

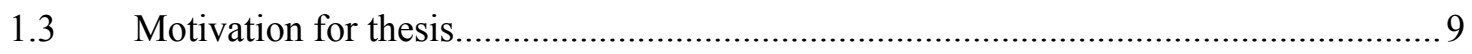

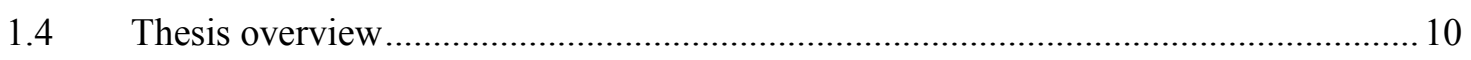

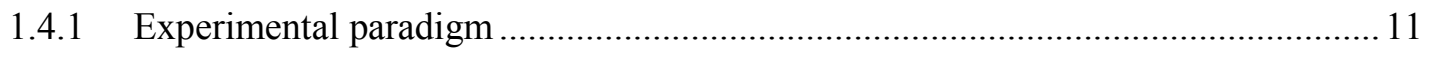

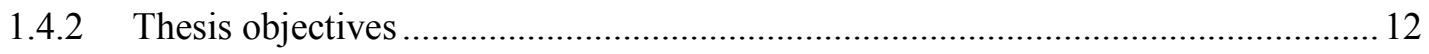

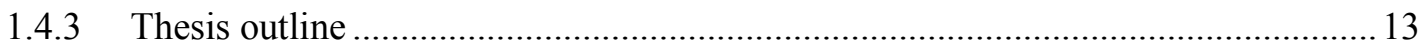

\section{Chapter 2: A brief review of disruptive coloration from ultimate and proximate}

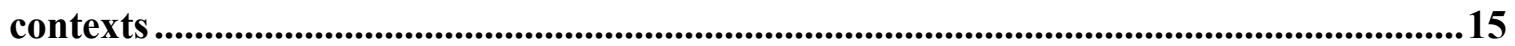

2.1 Disruptive coloration's ultimate context - evolution and function ............................. 16

2.2 Disruptive coloration's proximate context - reviewing visual and cognitive

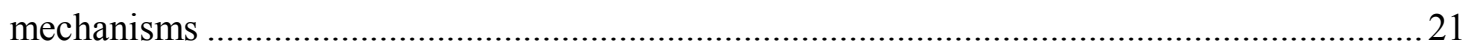

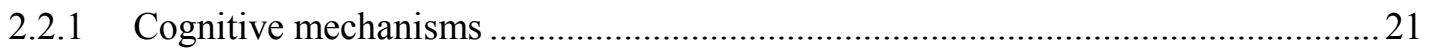

(a) Object recognition and the role of shape perception........................................... 22

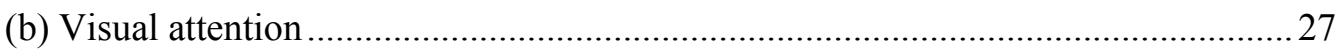




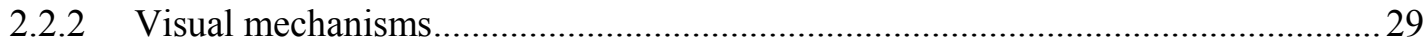

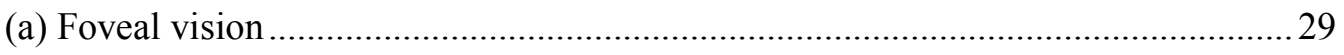

(b) Peripheral vision.....................................................

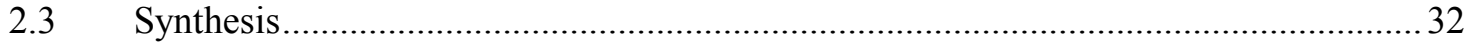

Chapter 3: Behaviourally mediated crypsis in two nocturnal moths with contrasting appearance ...................................................................................................................................33

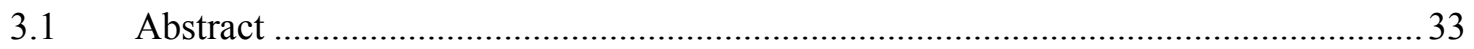

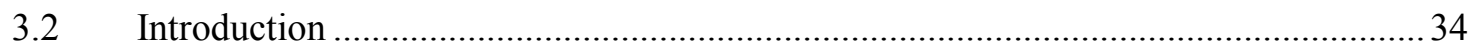

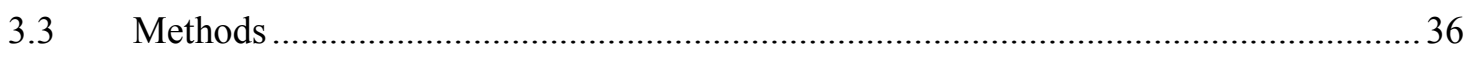

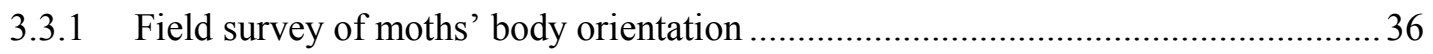

3.3.2 Human predator system: testing the effect of orientation on crypsis ...................... 37

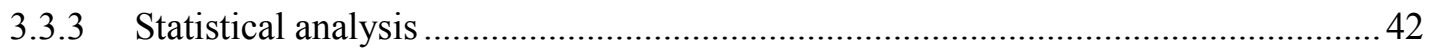

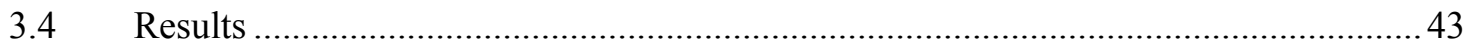

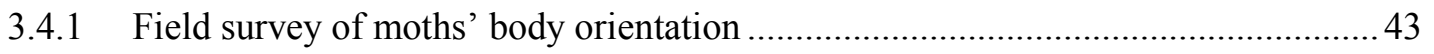

3.4.2 Human predator system: testing the effect of orientation on crypsis ...................... 45

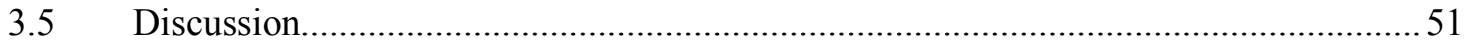

Chapter 4: Disruptive coloration and the role of shape perception in animal camouflage

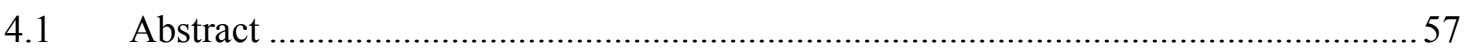

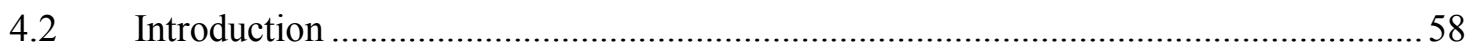

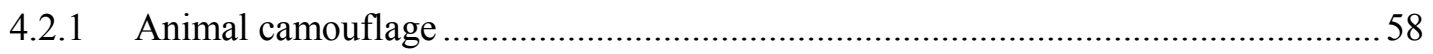

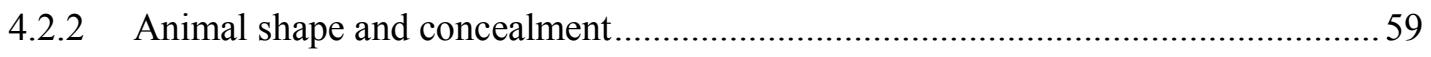

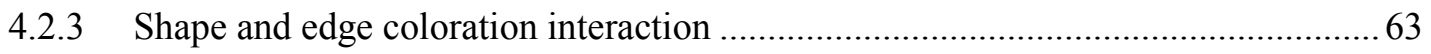

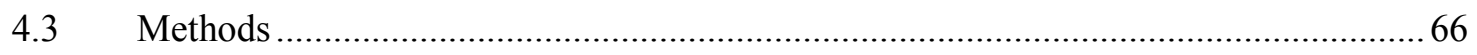

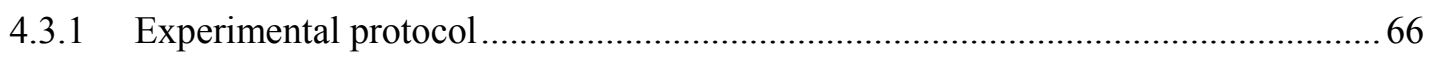

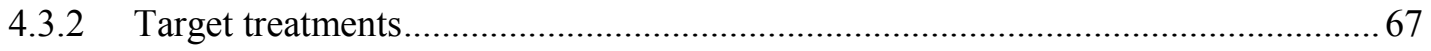




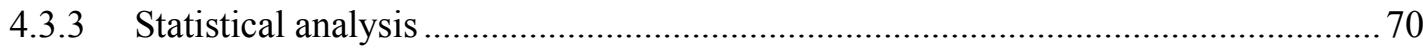

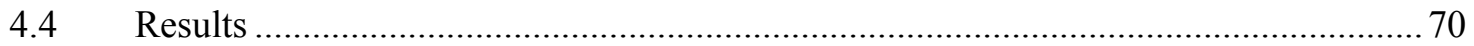

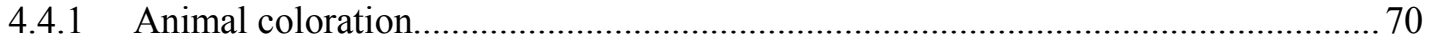

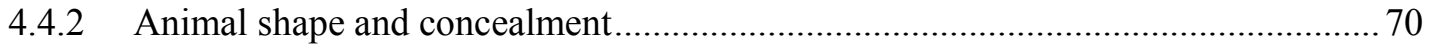

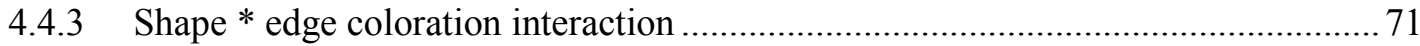

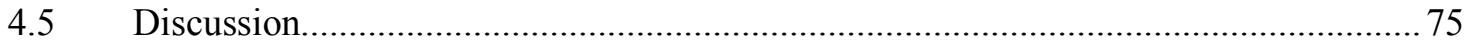

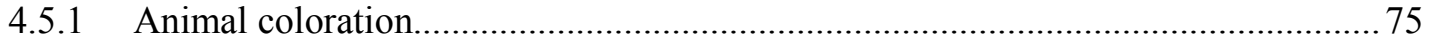

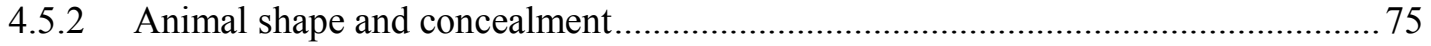

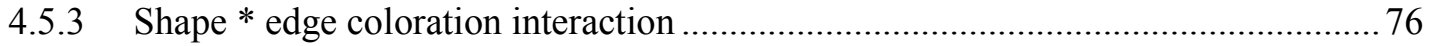

Chapter 5: Disruptive coloration and boundary visibility: Testing functional predictions

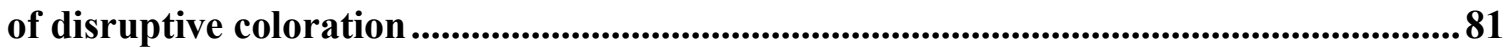

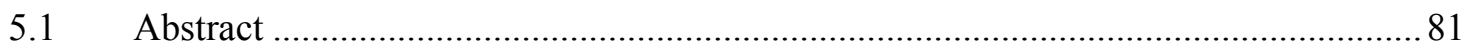

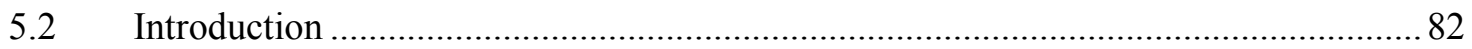

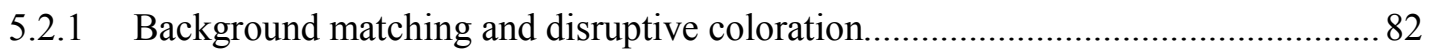

5.2.2 Boundary visibility is operationalized through transparency ................................. 85

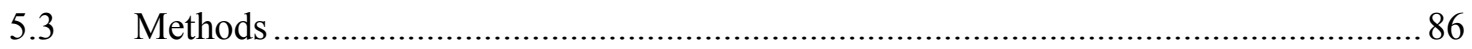

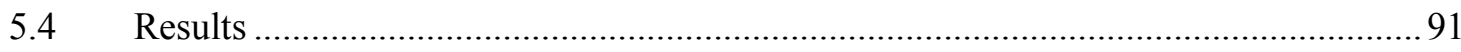

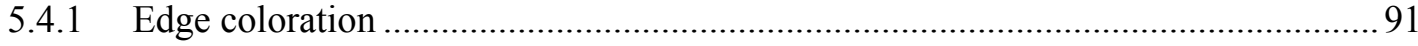

5.4.2 Animal boundary visibility and concealment.................................................. 91

5.4.3 Boundary visibility * edge coloration interaction ............................................. 91

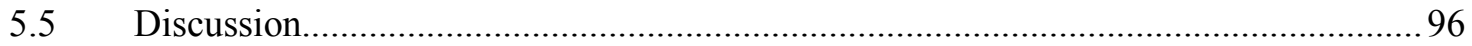

Chapter 6: Disruptive camouflage impairs object recognition ........................................ 101

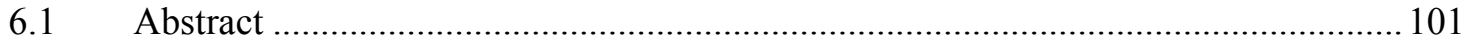

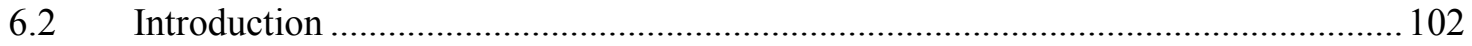

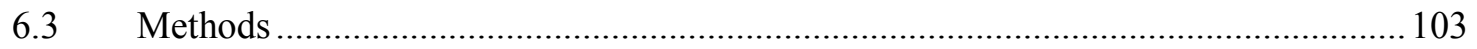

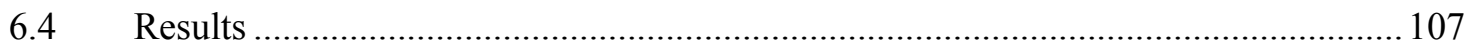




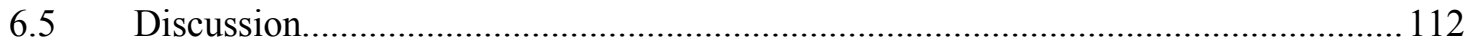

Chapter 7: General Discussion and Conclusions........................................................... 114

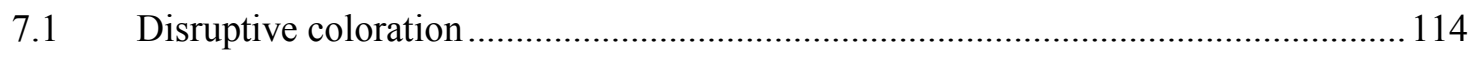

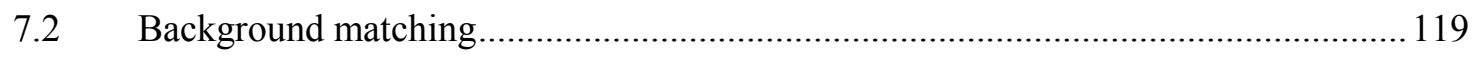

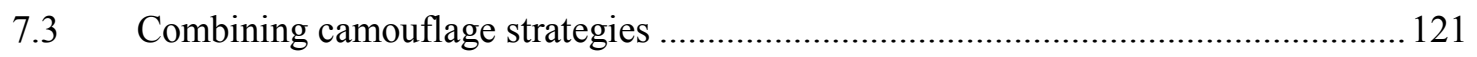

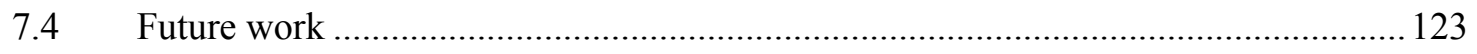

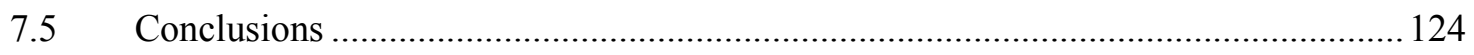

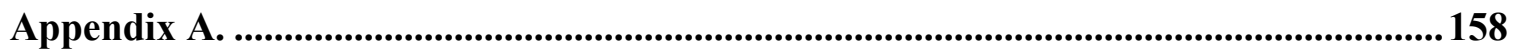

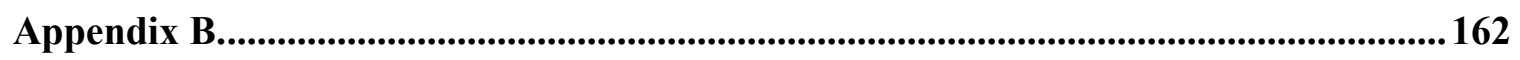

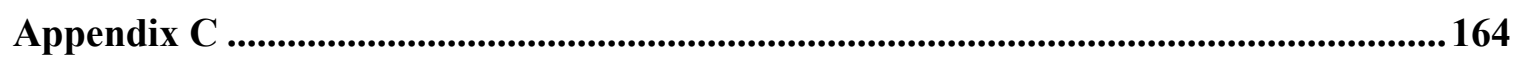

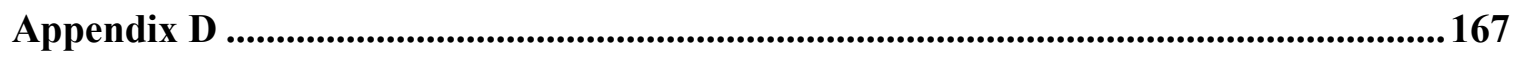

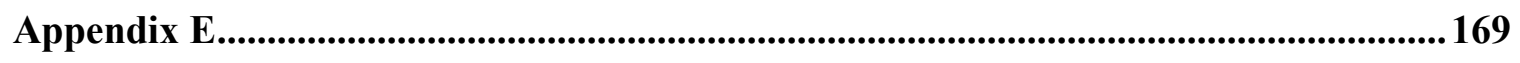




\section{List of Tables}

Table

Page

3-1 GLMs of arcsine transformed overall mean proportion missed (survivorship) per human subject for each moth species with three main effects (absolute moth orientation, tree rotation and tree) and all pairwise interactions.......................................

3-2 GLMs of square root, log-transformed detection time of each moth attacked, for each species and tree rotation with three main effects (absolute moth orientation, human subject and tree) and all pairwise interactions

4-1 Factors affecting survivorship of moth targets. The results of this logistic regression are presented. Here I control for the random factors of SubjectID, Tree background and TargetID. Cells reporting results are formatted such: Wald Chi-squared test statistic, degrees of freedom, and $p$-value. The column Full represents analysis of the full dataset, while columns Straight vs. Low and Straight vs. High subset the data to consider straight shape pair against on other each ruffled shapes. The sample size for each analysis were, Full $(n=4019)$, Straight vs. Low $(n=2600)$ and

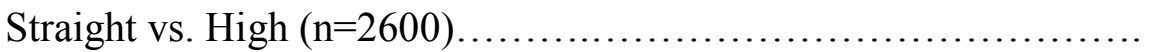


4-2 Factors affecting search time of moth targets. The results of this general linear mixed model are presented. Here I control for the random factors of SubjectID, Tree background and TargetID. Cells reporting result are formatted such: $\mathrm{F}$ test statistic, degrees of freedom and $p$-value. The column Full represents analysis of the full dataset, while columns Straight vs. Low and Straight vs. High subset the data to consider straight shape pair against on other each ruffled shapes. The sample size for each analysis were, Full ( $\mathrm{n}=2987)$, Straight vs. Low ( $\mathrm{n}=2017)$ and Straight vs. High $(\mathrm{n}=2018)$

5-1 Factors affecting both survivorship and search time of moth targets by human subjects. The results of the multivariate general linear mixed model are presented......................................

5-2 Factors affecting survivorship and search time of moth targets by human subjects. The results of two univariate general linear mixed model are presented, in separate columns with dependent variables as survivorship and search time

6-1 Testing the effects of the moth target's coloration properties on detection and recognition. Each data column shows the results of 
the fit of a separate general linear model. All fitted models included three target coloration properties as predictor variables (number of edge-intersecting patches, total proportion of targets dark area, and uniformity). These predictors were fitted using Type III sums of squares to control for the effect of correlated coloration properties.

The geometric means for the detectability and recognisability of each target were calculated across all 63 target presentations.

7-1 An extensive summary of empirical work done on disruptive coloration. Columns denote the types of evidence test with the symbols representing whether the predictions of disruptive

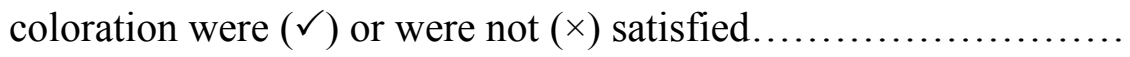




\section{List of Figures}

$\begin{array}{lll}\text { Figure } & \text { Page }\end{array}$

1-1 Photographic examples of edge coloration...................... 6

2-1 Photographs and illustrations of the different types of disruptive coloration.................................................... 18

2-2 Visual illusions of shape that are perceived by humans and nonhumans. (a) Kanizsa illusion involving perceptual grouping, and (b) a graph adapted from Zylinski et al. (2012) illustrating how the camouflage strategies of the cuttlefish are influenced by their shape perception of background.................................... 25

2-3 Illustrations of edge detection, contour integration, and contour completion....................................................

3-1 Photographs of the study species (a) C. cerogama and (b) E. intermediate, and the angular distributions of individual representatives found on trees in the field (c) Catocala spp. and (d ) Larentiinae ........

3-2 Mean proportion of moth targets missed ((a) C. cerogama and (b) E. intermediata) on (i) vertically and (ii) horizontally rotated trees 
according to absolute moth orientation

3-3 Mean detection time for moth targets ((a) C. cerogama and (b) E. intermediata) on (i) vertically and (ii) horizontally rotated trees according to absolute moth orientation.........................................

4-1 Three sphinx moths that show a diverse range of wing shapes

4.2 Graphical illustrations of how varying edge markings and targets' shape influences survivorship, according to conceptual models of how edge markings function

4.3 Factorial design of artificial moth target treatments, varying shape and edge coloration.

4-4 Edge intersecting patches correlated with (a) survivorship and (b) search time

5-1 Illustration of the 2-by-2 factorial design of target treatments

5-2 Edge intersecting patches correlated with (a) survivorship and (b) search time 
6-1 Photograph of eye tracker with a human subject.................... 106

6-2 Camouflaged moth-like target on tree background that is superimposed with eye tracking data showing the visual search path..................... 107

6-3 Detection probability of moth targets as a function of edge properties.

(a) Examples of moth targets. Edge-intersecting patches correlated with (b) mean survival and (c) mean search time..................

6-4 Number of edge-intersecting patches correlated against with the two metrics of recognition, (a) mean inspection time and (b) mean number

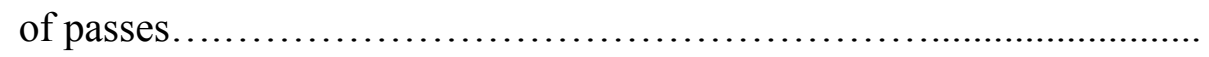




\section{List of Appendices}

Appendix

Page

Table A.1 Literature summary for background matching.....................

Table B.1 Literature summary for disruptive coloration

Table C.1 GLMs of survivorship for each moth species for relative moth orientation

Figure C.1 Survivorship of C. cerogama and E. intermediata per tree, for each of the eight possible orientations

Figure C.2 Colour images of $(a$ and $b)$ C. cerogama and ( $c$ and $d)$

E.intermediata presented in their ( $a$ and $c$ ) South and $(b$ and $d)$

West relative orientations.

159

Figure D.1 Photograph of a ghillie suit.

160

Figure D.2 Examples of targets of three different shapes, unfiltered and filtered to display only low spatial frequencies. 
Figure E.1 Examples of eye movements and visual search paths.

Figure E.2 Frequency distributions of the 63 moth targets depicting variation in (a) the number of edge patches, $(b)$ uniformity, and (c) proportion of surface area covered by dark patches...

Figure E.3 Edge-intersecting patches are positively correlated with breaking up a target's outline (estimated using the Canny edge detection algorithm) 168

Figure E.4 Relative abundance of targets' edge-intersecting patches compared to the backgrounds.

Figure E.5 Percentile of edge patches relative to background abundance plotted against (a) survivorship and $(b)$ search times.

Figure E.6 Percentile of edge patches relative to background abundance plotted against $(a)$ inspection time and $(b)$ number of independent passed overs

Figure E.7 Relationship between mean total inspection time and mean search time is mediated by disruption. 174 
Figure E.8 Scatter plots showing possible collinearity between the three target coloration properties.

Table E.1 Testing the effects of the moth targets' coloration properties on detection and recognition, when the edge patch abundance of each target was expressed as a percentile with respect to equivalent targets cut from the background...........................

Table E.2 Results of logistic regression testing for the effects of the target coloration on survivorship.

Table E.3 Generalised linear mixed-effects models (GLMMs) to test for the effects of target coloration on detection and recognition.

Table E.4 Disruptive coloration mediates the contribution of recognition to target detectability. A general linear model (type III SS) was fitted to test the effects of both inspection time (total time focusing in vicinity of target) and target colour properties (number of edgeintersecting patches, proportion dark area and uniformity) on the mean total search time (i.e. the time taken to discover target)......

Table E.5 Testing the effects of the number of edge patches quadratic term on moth targets' detections. Each data column represents the fit of a separate general linear model. All fitted models included three target coloration properties as predictor variables (number of edge patches, total proportion of targets covered by dark area, and uniformity), with the addition of number of edge patches quadratic term 


\section{Chapter 1: General Introduction}

"Concealment is an outward and visible sign of an inward ecological need, and that its function is directly concerned with the problem of survival in a dangerous world-a world in which hunger and murder are common-place, and one in which the most pressing business of life is the search for security and sustenance." (Cott, 1940)

\subsection{Animal camouflage and a century-old unanswered question}

Animal camouflage is the phenomenon by which animals conceal themselves in plain sight. Offensive camouflage allows predators to get close to their prey without being detected (which increases the predator's likelihood of a successful attack), whereas defensive camouflage allows prey to avoid detection by their predators (which increases a prey's survivorship and negates the need to use other more costly anti-predator escape strategies) (Cott, 1940, Ruxton et al., 2004b, Stevens and Merilaita, 2011). There is a strong selective advantage to being well camouflaged. Indeed, some of the first evidence for the neo-Darwinian synthesis of evolution was shown with observations of the melanistic camouflage of moths (Kettlewell and Conn, 1977, Kettlewell, 1956). Camouflage also plays an important role in human culture, with wide ranging applications in areas such as architecture, fashion, hunting, and the military (Behrens, 2002). Not surprisingly, camouflage research has a long and rich history, with prominent biologists (Darwin, 1859, Poulton, 1890, Cott, 1940, Wallace, 1889), artists (Thayer, 1909, Scott, 1961, Behrens, 2009) and computer scientists (Turing, 1952) coining and describing many camouflage strategies. 
Different camouflage strategies provide concealment through a variety of means. These strategies can operate through any of the sensory modalities (Ruxton, 2009), such as auditory, chemical, tactile, and visual. The focus of this thesis is visual camouflage, achieved through protective body coloration patterns. Visual camouflage is broadly achieved both by being indistinct from one's surroundings (i.e. background matching (Endler, 1984, Ruxton et al., 2004a, Poulton, 1890)) and by deceiving the eye of the beholder, through masquerading (Skelhorn et al., 2011, Skelhorn et al., 2010, Skelhorn and Ruxton, 2010, Skelhorn and Ruxton, 2013), countershading (Stoner et al., 2003, Rowland et al., 2007, Allen et al., 2012), polymorphism (Bond and Kamil, 2006, Pellissier et al., 2011, Wilson et al., 2007, Tsurui et al., 2010) or disruptive coloration (Stevens and Merilaita, 2011).

Before laying out the argument, motivation and objectives of this thesis, I briefly define below these animal camouflage strategies and describe their proposed functions. First, the most commonly used definition for background matching is when an animal's coloration ${ }^{1}$ resembles " a random sample of the background perceived by predators at the time and prey's age, and in the microhabitat where the prey is most vulnerable to visually hunting predators" (Endler, 1984). This implies that background matching is dependent on the visual appearance of the animal's surroundings (Poulton, 1890, Wallace, 1889, Darwin, 1859). This definition also emphasizes the importance of a sensory ecology approach (Endler and Basolo, 1998, Bennett and Cuthill, 1994); according to this view, because defensive camouflage has evolved as protection against visual perception by

\footnotetext{
${ }^{1}$ Throughout this thesis coloration is used to reference to both colour and textural patterning appearance.
} 
predators, it is essential to consider camouflage from the predators' perspective (i.e. account for the predators' colour vision and acuity). Background-matching animals are protected by diminishing their detectability through being indistinct from their background's luminance, colours and textures (see Appendix Table A.1). It is worth clarifying this definition, as Endler (1984) and subsequent researchers (Stevens and Merilaita, 2011) noted that background matching is enhanced when animals resemble common visual elements of the environment. ${ }^{2}$

Masquerade is defined as an animal's resembling a model inedible object (Skelhorn et al., 2010) and is distinct from mimicry because the model it is imitating is not aggressively protected (i.e. is neither aposematic or possesses a toxin or sting). Stones, twigs and bird feces are common natural objects used as models by masqueraders. Some limitations of masquerade are the size (Skelhorn and Ruxton, 2013) and density dependency of their models (Skelhorn et al., 2011).

Countershading occurs when there is a gradation of coloration from the dorsum to ventrum of an animal that obscures its self-shadowing (Poulton, 1888, Thayer, 1896). Since animals are three-dimensional in shape, overhead sunlight causes uneven illuminance; the dorsal side is brighter than the ventral side due to the shadow cast by the animal's body. These illumination gradients are a visual signature (Tankus and Yeshurun,

\footnotetext{
${ }^{2}$ It has long been speculated that background matching can be enhanced through resembling common visual elements. Appendix Table A.1 shows that there are surprisingly few empirical demonstrations of this intuitive concept: only five studies show that detectability varies between random samples of the background, and no studies show the ideal quadratic function of background abundance versus detectability (with the longest time to detect for animals matching the most common elements and diminished time to detect for phenotypes matching uncommon elements).
} 
2001) that are used by animals to recognize objects and even other animals. By masking this visual signature through the use of darker dorsal and lighter ventral pigmentation, animals are less recognizable and go undiscovered for longer periods (Rowland et al., 2007, Allen et al., 2012).

Polymorphism is where one species has multiple distinctive coloration variants (Poulton, 1890). In some cases, individuals with these visually different forms occupy identical ecological niches and associate with the same microhabitat; yet, surprisingly, they have evolved different camouflage solutions. Prey occurring in multiple, visually distinct forms forces predators to search for multiple, distinct prey items. This is cognitively challenging, resulting in the predator being less effective at detecting prey (Bond, 2007). Further, because of the increased cognitive burden placed on predators hunting for polymorphic prey, more attention is placed on hunting, and less invested in vigilance behaviours, which potentially places themselves at greater risk from their own predators (Dukas, 2004, Fernández-Juricic et al., 2011).

Lastly, disruptive coloration consists of markings that break up characteristic shapes (e.g. body outline) or other salient features (e.g. limbs or eyes) of animals. Characteristic shapes and outlines are important visual clues for animal recognition (Lloyd-Jones and Luckhurst, 2002, Lloyd-Jones et al., 2010), which are known to facilitate rapid animal identification (Elder and Velisavljevic, 2009). Disruptive coloration is proposed to visually fragment such features, thereby making them less recognizable. See Chapter 2 for a literature review of disruptive coloration. 
Although each of these camouflage strategies functions through different mechanisms, an animal can increase its camouflage by employing a mixture of camouflage strategies. While all of these strategies are interesting, disruptive coloration and background matching are of particular interest because disruptive coloration is reliant on background matching (Fraser et al., 2007), although the extent of this reliance remains uncertain (Schaefer and Stobbe, 2006). Because disruption and background matching work in tandem, they can be difficult to differentiate when categorizing an animal's camouflage. Since 1909, when Thayer proposed the camouflage strategy of disruptive coloration (Thayer, 1909), scientists have been trying to distinguish between disruptive coloration and background matching; this is required to show that disruptive coloration is a separate camouflage strategy with a specific function. To demonstrate this challenge, consider the edge-intersecting patches illustrated in Fig. 1.1; these could function to either resemble portions of an animal's background or disrupt its body outline. In this thesis, I will argue that specific evidence is required to understand animal camouflage and that background matching and disruptive coloration are distinct strategies. 

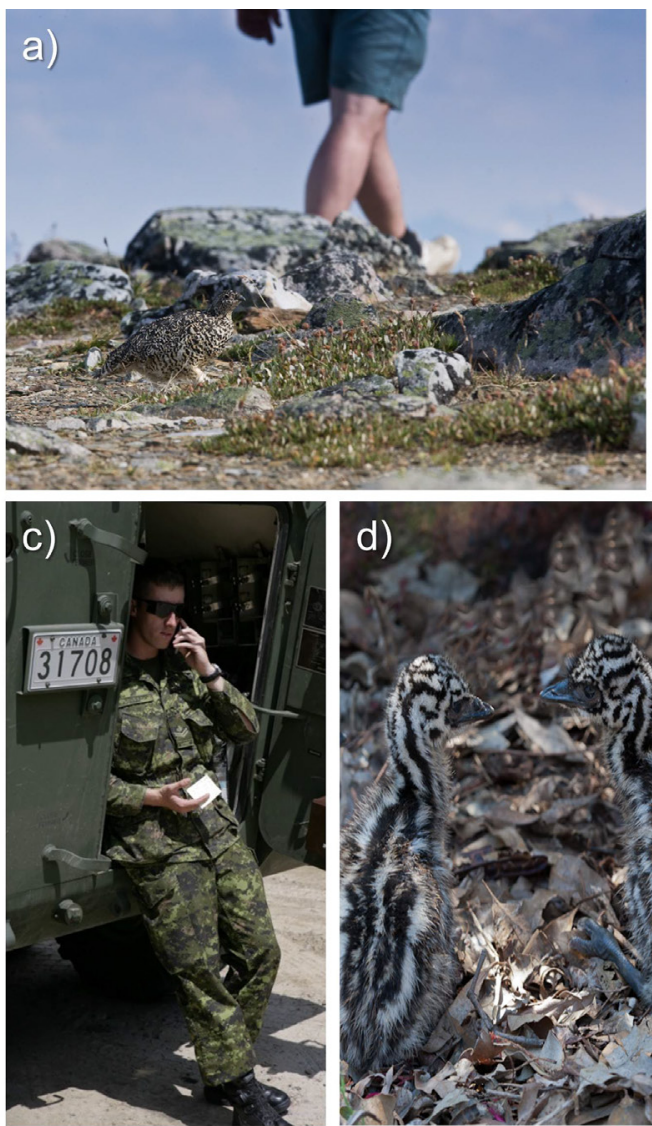
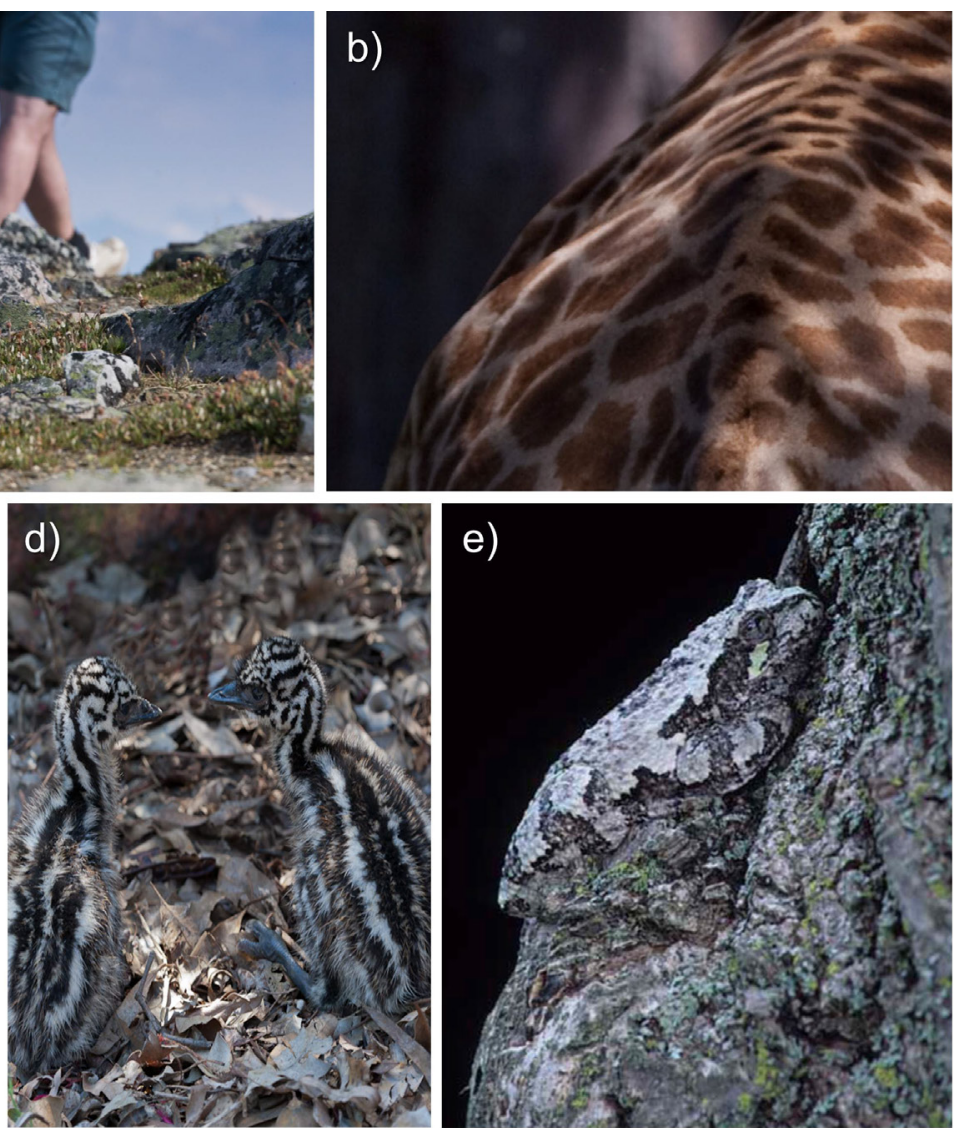

Figure 1.1: Do these edge patches contribute to camouflage through background matching or disruptive coloration? (a) White-tailed Ptarmigan Lagopus leucura, (b) Marai giraffe Giraffa camelopardalis tippelskirchi, (c) Canadian Disruptive Pattern (CADPAT), the Worlds first digital camouflage, (d) Emu chicks Dromaius noaehollandiae, $(e)$ tree frog Hyla versicolor. Photographs by Michael Webster $(a-d)$ and Micheal Runtz (e). 


\subsection{Categorizing animal colorations through their function}

How animal coloration operates is challenging to determine on visual appearance alone. Categorizing animal camouflage strategies using their visual appearance alone can lead to subjective or, worse, artificial conclusions (Stevens and Merilaita, 2011, Stevens and Merilaita, 2009). For instance, if a subjective approach is used to assign function to a red patch on a moth's wing, then one might conclude that it functions as a sexual signal when alternatively these markings might function as a warning signal for predators. Similarly, one might assume that a well-placed blotch on the edge of a moth's wing functions to disrupt the moth's characteristic triangular shape, when alternatively these markings might function to match the background. For instance, while a zebra's stripes have long been thought to be disruptive, there exists at least five alternate hypotheses that could also explain how the stripes function to protect the animal (Ruxton, 2002).

Like visual appearance alone, the combination of visual appearance and survivorship information is also an inadequate basis for inferring camouflage strategy. Just because an animal survives better with a particular colour patterning, this does not mean that camouflage explains its increased survival (e.g. the coloration might alternatively influence thermal properties (Stuart-Fox and Moussalli, 2009)) or possibly the pattern indeed serves to conceal but not for the reason assumed (i.e. the researcher infers one camouflage strategy, when an alternate is at work). Collectively, there are pitfalls when trying to establish functions for animal coloration based on visual appearance, even if it is combined with rate of detection. 
Because animal colorations are defined by their function (e.g. disruptive coloration is defined by break up the visibility of salient features, to impair recognition), it seems intuitive to categorize coloration using evidence about how they function. While a behavioural outcome (i.e. rate of mating, rate of detection, etc.) associated with an animal coloration may provide insight into a function, assessing function directly is clearly a superior way to distinguish between animal colorations (Stevens and Merilaita, 2009, Stevens and Merilaita, 2011, Cott, 1940, Cuthill and Troscianko, 2011, Caro, 2011). For instance, when differentiating between sexual signals and camouflage, one would expect the outcome of increased mating opportunities for the former and reduced discovery from predators for the latter. By contrast, if attempting to differentiate between animal colorations that have the same outcome (i.e. increased survivorship), but function differently (e.g. background matching versus disruptive coloration), measuring the behavioural outcome (increased survivorship) in isolation is not sufficient to distinguish between them.

Here, I take the latter perspective in arguing in this thesis that evidence of how body colour markings function is required to address the century-old challenge of distinguishing disruptive coloration from background matching. Both background matching and disruptive coloration have the same behavioural outcome of reducing prey discovery by predators, although they differ in how they function to achieve this. Consideration of visual appearance characteristics and corresponding enhanced survivorship are necessary, but insufficient, to distinguish between these two camouflage strategies. Additional evidence is also required to determine the functional differences between background matching and disruptive coloration (Stevens and Merilaita, 2011, Stevens and Merilaita, 2009). For 
instance, if edge markings act as disruptive coloration, then the following functional predictions can be made: (1) edge coloration increases concealment that is dependent on visibility of boundary and shape properties, (2) edge coloration breaks up an animal's outline, and (3) edge coloration impairs object recognition. These predictions describe how edge markings should behave if they operate as disruptive coloration. Further, evidence of these predictions - unlike an association with lower detection rates alone — can allow us to attribute function to an animal coloration. I use a function-centric approach to demonstrate that edge markings operate through disruptive coloration, a distinct camouflage function from background matching.

\subsection{Motivation for thesis}

My thesis research is timely because no study to date has satisfactorily tested whether disruptive coloration is a functionally distinct form of camouflage from background matching (see Chapter 2.1 and Appendix Table B.1). From an evolutionary ecology perspective, it is desirable to assay animal coloration such as disruptive coloration; for instance, the iconic animal coloration of zebras, giraffes, tigers and tapirs (to name but a few) may or may not be attributed to disruptive coloration. Clearly, testing how the body coloration patterns of all these animals function is beyond the scope of one doctoral dissertation. Further, given the discipline's current state of understanding of disruptive coloration, it seems more productive to develop novel methods to distinguish background matching and disruptive coloration, which others can later use to evaluate speciesspecific cases where disruptive coloration has been proposed. It is the goal of this thesis to test whether disruptive coloration operates separately from background matching, and 
to provide methodologies that will be applicable for future work to test if animal coloration functions disruptively.

My investigating disruptive coloration here is further motivated by its influences on predator-prey interactions, micro-habitat choice and whether different animals might be habitat specialists or generalists (Merilaita et al., 1999). For instance, a backgroundmatching strategy might perform poorly on visually heterogeneous environments, whereas disruption has been suggested to overcome this conventional limitation. Research into camouflage also has real-world applications (e.g. the design of military uniforms and vehicles), but here I focus on more fundamental research questions. For instance, camouflage — and disruptive coloration in particular — is an interesting lens through which object recognition can be explored, and is a research question of interest to biologists, psychologists, vision scientists and computer scientists alike.

\subsection{Thesis overview}

In this General Introduction, I have so far defined several camouflage strategies, described the challenge involved in separating disruptive coloration from background matching, and argued that predictions about how camouflage functions are an important — and often overlooked — form of evidence in distinguishing between camouflage strategies. For the remainder of this General Introduction, I will introduce the experimental paradigm used, state the objective of this thesis, and present the key predictions under experimental test in Chapters 3-6. 


\subsubsection{Experimental paradigm}

For most of the experiments described in my thesis, I used human subjects and artificial moth-like targets of a computer screen as a model predator-prey system, and a human visual search task (i.e. a human foraging on computer screen) to measure concealment efficacy of artificial moth-like targets. The approach of using human visual search to test concepts of anti-predator defense function and evolution is a well-established experimental paradigm (Webster et al., 2009, Penney et al., 2012, Fraser et al., 2007, Beatty et al., 2005, Sherratt and Beatty, 2003, Knill and Allen, 1995, Bain et al., 2007, Karpestam et al., 2012). Several studies have shown that human visual foraging is similar to other terrestrial vertebrate predators in search tasks (Fraser et al., 2007, Bain et al., 2007). Although it is possible that disruptive coloration's reliance on visual mechanisms of shape perception might differ between humans and non-humans, I believe that this is unlikely because, while vertebrate species have anatomically different brains, the process of shape perception is evolutionarily conserved across them (Chapter 2.2.1a). It is therefore assumed that findings from such human-based foraging tasks will provide insights into the function of disruptive coloration in nature.

There are several benefits to using human foraging experiments. First, the mechanisms that underlie human visual perception are much better understood than those of most other vertebrates. Subsequently, this work can be related to concepts in psychology, neurobiology and vision science, which is helpful when interpreting novel results. Second, human laboratory-based experiments can be well controlled and therefore provide a high degree of reproducibility between research institutes. Finally, there are some technical and logistical aspects of working with humans that permit the 
use of methods that would be more difficult to use with other organisms. For instance, in Chapter 5, eye tracking is conducted during visual search tasks using human subjects. Eye tracking is difficult to achieve in the laboratory and has yet many more challenges in the field (Hayhoe and Ballard, 2005, Evans et al., 2012), especially on non-humans (but see (Einhauser et al., 2009, Ohayon et al., 2008, Kjaersgaard et al., 2008)).

\subsubsection{Thesis objectives}

The general objective of this thesis is to assess how camouflage coloration functions, with an emphasis on testing whether disruptive coloration distorts outline visibility through breaking up shape (Chapter 4) and boundary (Chapter 5) properties, which in turn impair object recognition (Chapter 6). Taken together, this thesis explores whether disruptive coloration is a specific camouflage strategy that is functionally distinct from background matching. 


\subsubsection{Thesis outline}

Before presenting the component data chapters of the thesis, I provide in the next chapter (Chapter 2) a more detailed literature review of disruptive coloration and background information on the mechanisms that underlie visual search.

In Chapter 3, I test the prediction that moth orientation behaviour provides concealment. Several moth species in the field were found to exhibit non-random resting orientation on tree trunks. Humans were set the task of foraging on computer screens for moths presented in different orientations. It is predicted that the lowest detection rates during computer trials should be when moth body orientation aligns with the observed preferred orientation behaviour on moths in nature. Further, the detectability of moths at different orientations is predicted to depend on the rotation of tree backgrounds. This chapter also provides support for the proposition that human foraging experiments provide biologically meaningful insight into animal camouflage. Chapters 4-6 use this human foraging experimental paradigm as well to address how edge coloration contributes to camouflage and to test predictions relating to the function of disruptive coloration.

Chapter 4 tests the key assumption that disruptive coloration affects shape perception. It is predicted that the benefits of disruptive edge coloration would be high for shapes with straight boundaries, but would diminish with shapes that are curvilinear. Using a factorial design, edge coloration and shape were varied to test for this interaction. 
Chapter 5 extends the reasoning of Chapter 4, that an interaction effect can diagnose disruptive function. This chapter focuses on testing the key assumption that disruptive coloration affects boundary perception. I operationalize boundary visibility by varying the target's boundary (control versus translucent). It is predicted that disruptive edge coloration will interact with boundary visibility.

In Chapter 6, I test whether edge coloration function (explored in Chapters 4 and 5) operates by impairing recognition. This is essential evidence required to claim that disruptive coloration has a specific camouflage function through degrading object recognition, rather than an artefact of background matching. Using eye-tracking technology, the foveal eye movements of human subjects were recorded while they searched for artificial moths with varying amounts of edge-intersecting patches presented on a large television screen. It is predicted that moth-like targets with more edgeintersecting patches will be inspected by foveal vision for longer before being identified, and be passed over unidentified more frequently.

Finally, Chapter 7 provides a General Discussion. This closing chapter summarizes the main findings of my thesis research, discusses the implication of these findings, and suggests future research directions. 


\section{Chapter 2: A brief review of disruptive coloration from ultimate and proximate contexts}

In this chapter, I provide both a literature review of disruptive coloration, an interdisciplinary subject, and necessary background information on the cognitive and visual proximate mechanisms that underlie this phenomenon, thus considering some of Tinbergen's four questions about animal behaviour (Tinbergen, 1963). Most of the empirical work on disruptive coloration — similar to this thesis's approach—is from a behavioural ecology perspective, addressing ultimate questions about the evolution and function of disruptive coloration.

Whilst in recent years there has been a growing interest in disruptive coloration in animals, there is still limited functional evidence that it is a distinct camouflage strategy. The artist Abbott Thayer first suggested that disruptive camouflage could be achieved via high-contrast colorations that break up an object's outline, thereby making the object more difficult to visually recognize, regardless of how well the colour markings match up with the background (Thayer, 1909). Since then, the existence of disruptive coloration as a camouflage strategy has been somewhat controversial. Historically, Thayer's principles of animal camouflage have received much attention as a result of the public debate of Darwin's theory of evolution. Notably, Franklin D. Roosevelt, an avid ornithologist and former president of the United States, was an open critic of the concept of disruptive coloration (Barbour, 1913, Allen, 1912) and the theory of evolution. It has been suggested that Thayer made his scientific theory less palatable to an audience of scientists by his eccentric artist's temperament (Behrens, 2002), a lack of experimental tests 
(Kingsland, 1978) and a dismissive attitude toward scientists (Barbour, 1913). Following Thayer, it was the British zoologist (and artist) Hugh Cott who succeeded in popularizing disruptive coloration as a camouflage strategy in the scientific community (Cott, 1940). Since then, disruptive coloration has been used to classify a wide array of animals' coloration patterns, but has remained untested for another 60 years, but see (Silberglied et al., 1980). Caro (2009) stated "Currently, there is no firm evidence for disruptive coloration in mammals", and this would seem true for all taxa.

In order to assay if disruptive coloration functions as a distinct camouflage strategy (the objective of this thesis), it is critical to outline the implicit assumptions of the visual and cognitive mechanisms that disruption is proposed to operate through. This is because much has been revealed about the workings and limitations of the mechanisms facilitating visual search since Thayer (1909) introduced the concept of disruptive coloration. By the end of this chapter, it will be clear how this thesis contributes to the current literature of disruptive coloration and is based on a grounding in the mechanisms of visual search.

\subsection{Disruptive coloration's ultimate context - evolution and function}

The broad-scale phylogenetic approach to studying the evolution of disruptive coloration has yielded limited insight into the seemingly widespread strategy of disruption (Caro, 2011). In recent years, there have been advances in deriving insights into phylogenetic pathways and ancestral selection pressures on animal traits, such as their coloration, by mapping traits onto molecular phylogenies (Paradis, 2006). Such research provides compelling evidence for camouflage strategies, such as countershading (Allen et al., 
2012, Santana et al., 2011) and background matching (Allen et al., 2011, Caro, 2011, Caro, 2005, Caro et al., 2012). A noteworthy drawback of these phylogenetic comparative studies, however, is the reliance on categorising camouflage strategies subjectively by their visual appearance (Stevens and Merilaita, 2011, Stevens and Merilaita, 2009). The appropriateness of classifying animals' coloration by their visual appearance depends highly upon the type of camouflage being categorised (e.g. countershading and background matching lend themselves to this approach). From visual appearance alone, it is currently challenging to distinguish between disruptive coloration and background matching; it seems inappropriate to classify disruptive coloration by merely the presence or absence of high-contrast blotches or stripes (since these markings might function to break up an outline or blend into a non-uniform background). Caro et al. (2005) suggest that the lack of success in studying disruptive coloration's evolution is in part due to the limitations of visual appearance classification and an apparent absence of predictions that relate directly to the function of disruptive markings, rather than simply their visual appearance. This provides motivation to explore disruptive coloration's function and develop testable predictions.

Hugh Cott's monograph (1940) laid the foundation of disruptive coloration in the scientific realm, and provided many examples of animal coloration that might function as disruptive. His book proposed a collection of disruptive coloration principles that he speculated might distort object shape and therefore impair object recognition (Cott, 1940). Some of these putative disruptive markings are: edge-intersecting patches that interfere with boundary detection to mask outlines; coincidental disruption, where high- 
contrast markings on adjacent body limbs align through behavioural posture to create a false boundary that is more visible than the animal's limbs; face disruption, where facial features are obscured by high-contrast coloration; and disruptive stripes that break up the uniformity of a shape, making it appear disjointed (Fig. 2.1). Whilst Cott set out a framework in which disruptive coloration role in camouflage is distinct from background matching, he presented no empirical work to support his hypotheses. Since Cott's era, the concept of disruptive coloration has permeated both natural history literature and popular culture at large, but still no work has rigorously tested its functional assumptions- that distorting outline or shape perception adversely affects animal recognition and increases camouflage (i.e. decreases detectability). 
a)
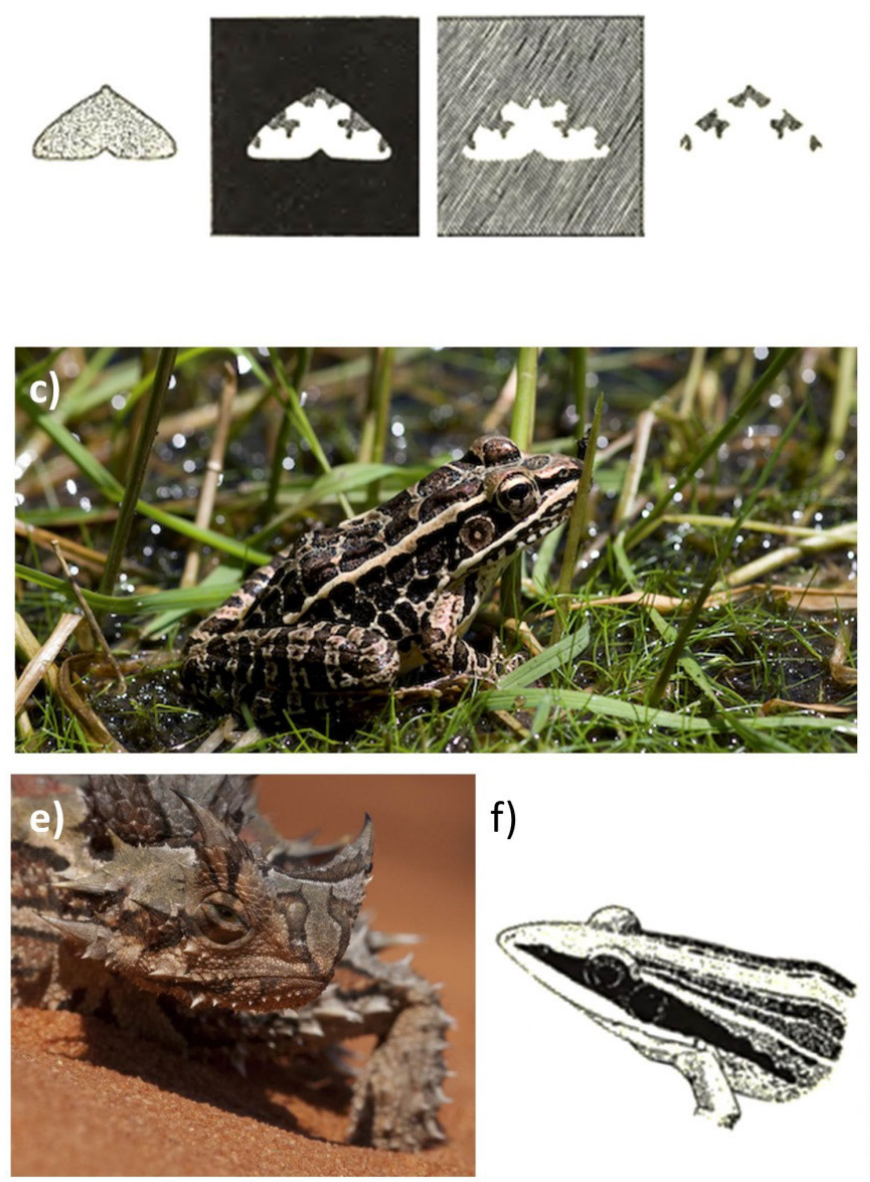

f)
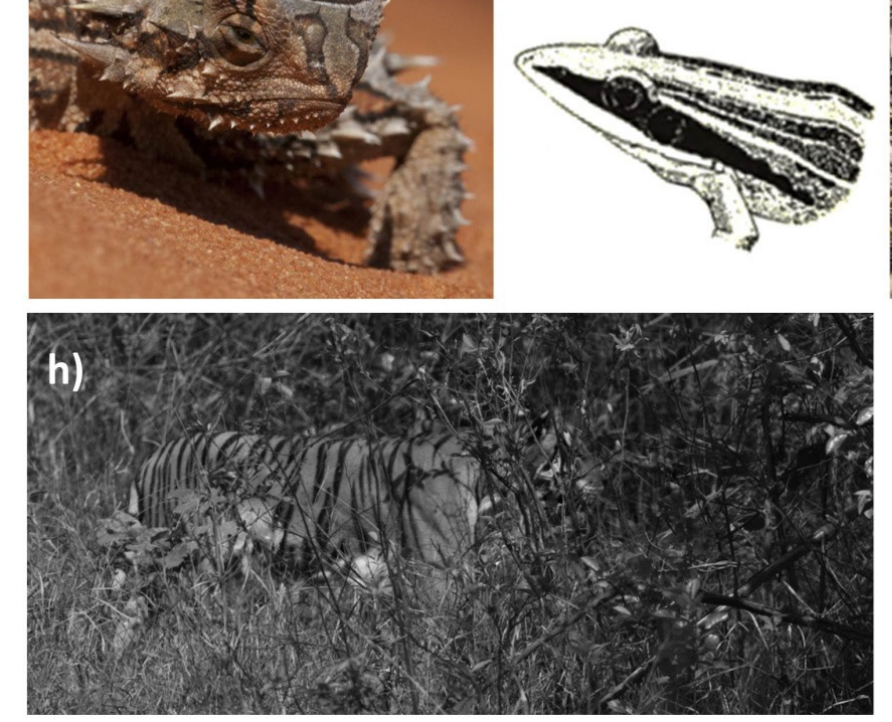

d)
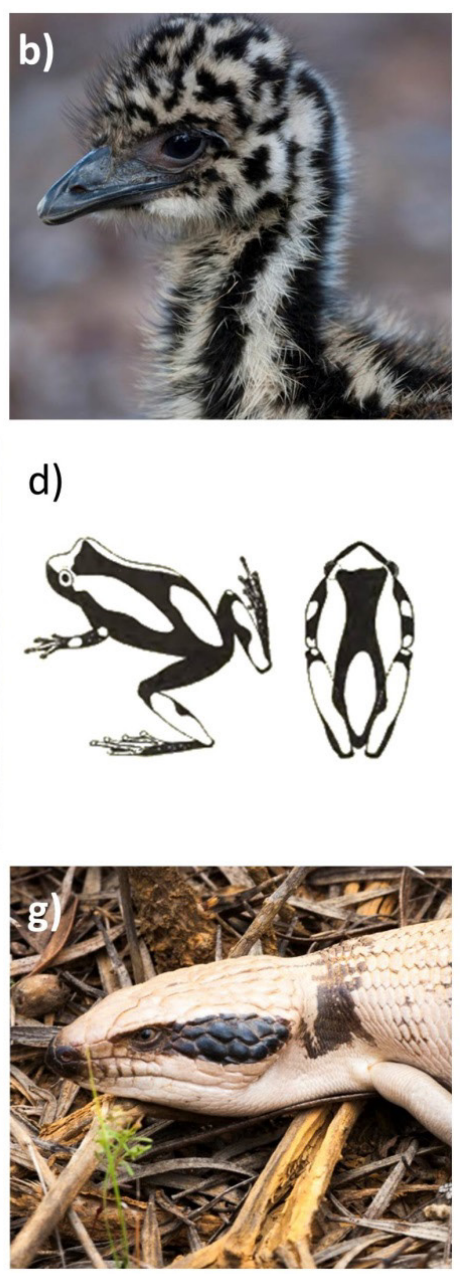

i)

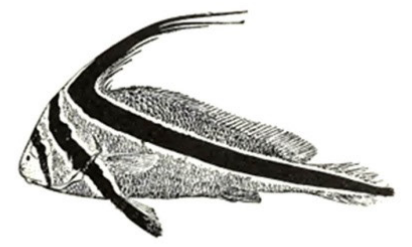

Figure 2.1: Examples of disruptive coloration. Outline disruption in $(a)$ moths and $(b)$ an emu chick Dromaius novaehollandiae. Coincidental disruption in $(c)$ a European marsh frog (Pelophylax ridibundus) and (d) a South American tree frog (Hyla leucophyllata). Face disruption in (e) a thorny devil (Moloch horridus), $(f)$ a frog (Rana stenocephala) and $(g)$ a western blue tongue skink (Tilqua occipitalis). Disruptive stripes $(h)$ a tigers (Panthera tigris) and (i) a Jack knifefish (Eques ianceolatus). Illustrations by Cott (1940). Photographs by Michael Webster. 
In recent years many empirical studies have tested for disruptive coloration, but they often failed to provide direct evidence for its function through the breaking up of outlines. The first such study was by Cuthill and colleagues who showed, in a pioneering field experiment, that artificial prey targets with contrastingly-patterned edges survived longer in the field under predation than targets without such edge markings (Cuthill et al., 2005), a result that was subsequently replicated in the laboratory using human subjects as predators (Fraser et al., 2007). Subsequently, several studies have assessed putative disruptive coloration using many experimental paradigms. The most common approach of these studies has been to test disruptive coloration by comparing between treatments that are approximately equivalent in their background matching, but varying the presence/absence of edge markings. If the treatment with edge markings enhances survivorship, it is inferred that edge markings act to disrupt edge information and increase survivorship. There are two limitations with this approach. First, it assumes that background matching is similar between all treatments, but this is rarely the case; by changing edge markings (and associated internal markings) the extent of background matching is also influenced and therefore is not controlled for. Second, by only measuring survivorship, this approach fails to directly test if edge coloration affects outline and shape perception. While association of edge markings with increased survivorship is a necessary prediction of disruptive coloration, this relationship is not sufficient to show a unique functionality for outline disruption. It therefore remains uncertain as to whether these studies in fact demonstrate that edge markings functionally act as disruptive coloration, as they claim, or if these findings are an artefact of background matching. Indeed, as Cuthill and Troskianko (Cuthill and Troscianko, 2011) themselves acknowledge, for disruptive coloration to work in the manner prescribed, then "object recognition must be impaired or the theory fails". 
The key test of the hypothesis of disruptive coloration is whether enhanced concealment can be directly attributable to changes in outline or shape perception when background matching is controlled for. To my knowledge, Stevens and Cuthill (2006) provide the only evidence to date that edge markings affect outline appearance by quantifying edge detection, but unfortunately this study does not adequately control for background matching. Using the Hough transformation edge detection algorithm, Stevens et al. (2006) tested if artificial moth-like targets, with or without edge-intersecting patches, differed in their outline detectability. Interestingly, they showed that targets with edge patches had outlines that were less detectable to computer vision algorithms. An over-simplified assumption they make is that the low-level process of edge detection corresponds directly to biological perceived differences in recognisability. After edge detection, there are many 'downstream' processing steps for object recognition (i.e. such as contour integration, contour completion and identification, which will be discussed in the next section Chapter 2.2.1a). The combination of using edge detection as a proxy for recognition and not measures of rate of target detection limits the insights into disruptive coloration from this. Of more concern should be the latter study's control for background matching. Two treatments of edge coloration (with and without edge-intersecting patches) were used, which these authors claim are equally background matching and only differ in their edge patches (which are putatively disruptive). For these two treatments to be equally background matching, however, it is insufficient that they are both sampled from the same background. Rather, it must be shown that they are equally representative of the background. Ultimately, the authors' scheme of selecting targets could have sampling bias, with their disruptive treatment likely to have patterning that is more 
representative of the background (as samples of the background with no edge-intersecting patches are uncommon, see Chapter 6). Therefore, whether survivorship differences between treatments with and without edge-intersecting patches are due to edge coloration or are an artefact of background matching remains uncertain. Thus, Stevens and Cuthill (2006) show that survivorship increases for targets with edge coloration, but this study is insufficient to demonstrate that increased target survivorship is due specifically to outline break up because of the confounding effect of background matching.

\subsection{Disruptive coloration's proximate context - reviewing visual and cognitive mechanisms}

\subsubsection{Cognitive mechanisms}

Because this thesis aims to use a functional approach to test for disruptive coloration, it is important to summarize here its underlying visual and cognitive mechanisms. Not only do these principles allow assessment of whether impaired object recognition, achieved independent of background similarity, could reduce animal detection, but also help explain why such mechanisms are commonplace across diverse taxa-thereby accounting for the apparently widespread occurrence of disruptive coloration in nature.

The hypothesis of disruptive coloration makes several assumptions about how visual and cognitive mechanisms work; namely that shape perception contributes to object recognition and degrading shape information can impair object recognition. Further, animal markings that are visually dissimilar to the background can increase 
camouflage if these markings sufficiently degrade the ability of predators to identify their prey. Surprisingly, for an interdisciplinary topic of such interest, there has been little synthesis of vision science, computer science, neurobiology and comparative psychology research in the context of camouflage (but see (Troscianko et al., 2009)). In the following sections, I first consider if the cognitive mechanisms utilize shape information for object recognition. Then I consider how aspects of vision impact shape perception and discuss implications for disruptive coloration.

\section{(a) Object recognition and the role of shape perception}

In order to outline the mechanism through which visual camouflage acts, it is important to briefly introduce the basic concepts of cognitive processing. The brain functions via interconnected neurons, which respond to visual stimulation through synaptic communication. The area of the brain that first processes visual information is the visual cortex. Hubel and Wiesel (1968) first showed that the vertebrate visual cortex has four types of neurons: concentric, simple, complex and end-stopper (Hubel and Wiesel, 1968)). Since then, many neurophysiological studies have suggested that the visual cortex has a hierarchical structure, with later stages increasing in complexity of processing, receptor field size, and neuron selectivity (Rust and DiCarlo, 2010). Across these regions of the visual cortex, the cognitive processes for object recognition that take place in sequence are (i) detection of features, (ii) grouping features and (iii) object identification.

Biological and computer vision both rely on edge detection as the first processing stage for object recognition (Bergevin and Levine, 1992). Edges imply basic parts of an

object and its spatial structure. Low-level filtering provides edge detection information by 
identifying spatial contrast, which is indicative of an object's boundaries. Some neurons in the visual cortex are specialized to identify visual signatures associated with object boundaries, whereby luminance and colour change invoke a reaction by simple and complex cells, whereas changes in background structure (i.e. seen when an object is partially obscured) stimulate end-stopper cells. By integrating different types of edgeboundary cues, edge detection is improved (Heitger et al., 1998). Whilst edge detection can be easily approximated (i.e. using computer vision algorithms (Wang et al., 2006, Stevens and Cuthill, 2006); see Chapter 5), this is just the first step of visual processing towards shape identification.

The second stage of object recognition is contour integration, whereby edge information is assimilated into shapes by the mechanism of perceptual grouping. Perceptual grouping is the task of assigning which visual elements make up objects in the scene, a phenomenon that is well documented in humans (Wertheimer, 1923, Wagemans et al., 2012a, Wagemans et al., 2012b, Helson, 1933). Perceptual grouping is an important process in shape perception, a by-product of which is visual illusions of shape (Halko et al., 2008) (Fig. 2.2a). Such visual illusions of shape, caused by perceptual grouping, are found in a wide range of animals (mammals (Spinozzi et al., 2009, Vonderheydt et al., 1984, Fagot and Tomonaga, 2001), birds (Nieder and Wagner, 1999, Zanforlin, 1981), fishes (Wyzisk and Neumeyer, 2007, Sovrano and Bisazza, 2008) and insects (Horridge et al., 1992)). This suggests that both humans and non-human vertebrates share an evolutionarily-conserved mechanism of perceptual grouping that underpins shape perception. Aggregation of detected edges by perceptual grouping is not an infallible means of pairing an object with its possible boundaries. Indeed, shapes with curvatures or many small sides are inherently more difficult for perceptual grouping (Kennedy and Domander, 1985, Panis et al., 2008). Consequently, biological vision can process 
straight-line boundaries, with low spatial frequency visual cues, more rapidly than curvilinear edges, thereby facilitating fast global shape recognition (Panis and Wagemans, 2009). It is not just an object's physical shape that affects shape perception; its surrounding environment is clearly also important and influential.

The task of object recognition is rendered even more challenging when large portions of an object's edge are hidden from view (Elder et al., 2003) and consequently observers are required to estimate where the missing contours should be. Incomplete object outlines (that are not found by edge detection and subsequently omitted from grouping edges fragments) is particularly common in natural scene with camouflage animals. An animal's outline can be hidden from view by either insufficient animalbackground contrast as perceived by the receiver ${ }^{3}$ (Marr and Hildreth, 1980, Stevens and Cuthill, 2006) or partial occlusion of the animal by foreground objects, obscuring portions of its boundaries from sight (Tvardikova and Fuchs, 2010). To combat incomplete boundary information, object recognition has been shown to 'fill-in' the missing pieces to estimate object shape (a process called contour completion; Fig. 2.3) (Anderson et al., 2002, Kellman and Shipley, 1991, Anderson, 2013). Because decision making during instances of incomplete visual information is a ubiquitous problem, it is not surprising that contour completion is another common mechanism of shape perception shared by humans and non-humans (mammals (Barbet and Fagot, 2011, Parron and Fagot, 2007, Deruelle et al., 2000, Sato et al., 1997, Kurylo, 2008, Fujita, 2001, Kanizsa et al., 1993), birds (Nakamura et al., 2010, Nagasaka et al., 2005, Cavoto and Cook, 2006, Regolin and Vallortigara, 1995) and fishes (Truppa et al., 2010, Sovrano and Bisazza, 2008), but see (Burke et al., 2001)).

\footnotetext{
${ }^{3}$ Receiver' is a term from the field of sensory ecology, denoting the observer.
} 
a)

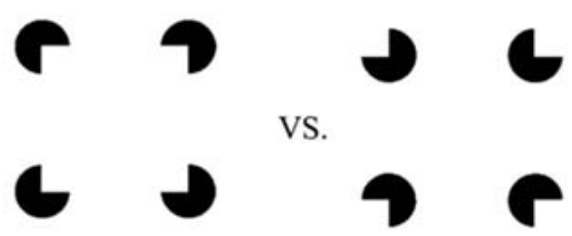
V) Visual elements that appear as
large shapes elicit high contrast camouflage response

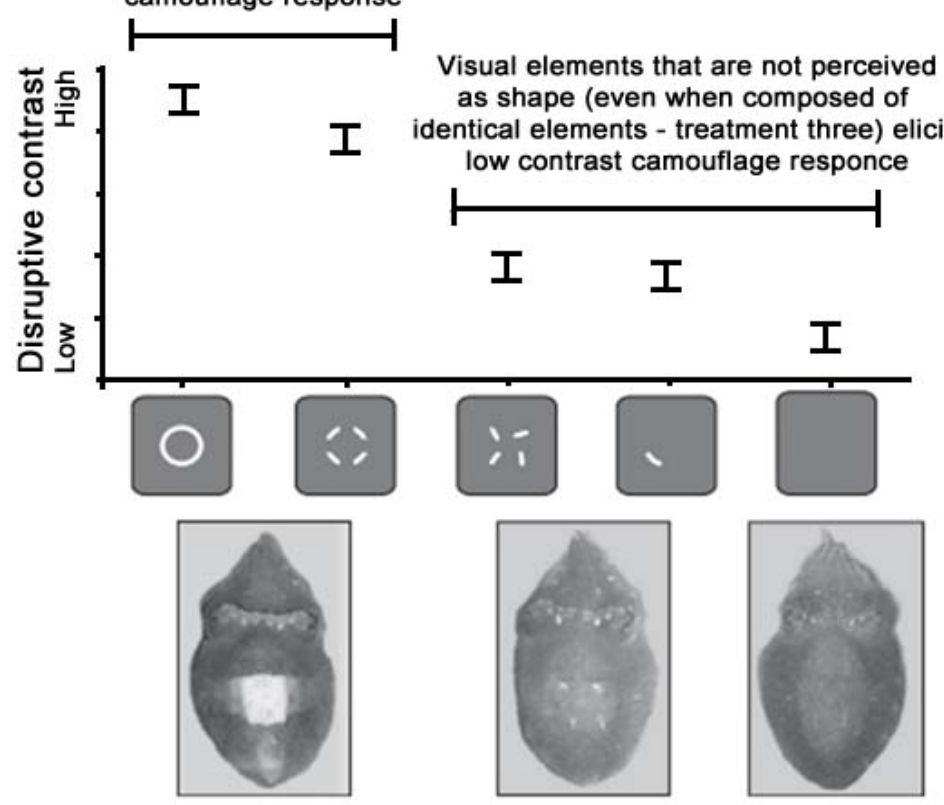

Figure 2.2: Visual illusions of shape that are perceived by humans and non-humans.

Such fundamental aspects of shape perception by cognitive systems are important in the context of disruptive coloration. (a) Kanizsa illusion where shape perception is affected by perceptual grouping, (b) Graph adapted from Zylinski et al. (2012) illustrating that cuttlefish dynamically express camouflage strategies in accordance to shape perception of background. There were five background treatments presented, one plain and four with repeated visual elements (a circle, four lines arranged like a circle, four lines arranged randomly [not like a circle] and one line). Interestingly, cuttlefish camouflage choice was consistent for backgrounds with circles and broken up circles, suggesting that cuttlefish use shape information for camouflage decision making and that contour completion may be present in this invertebrate species. 

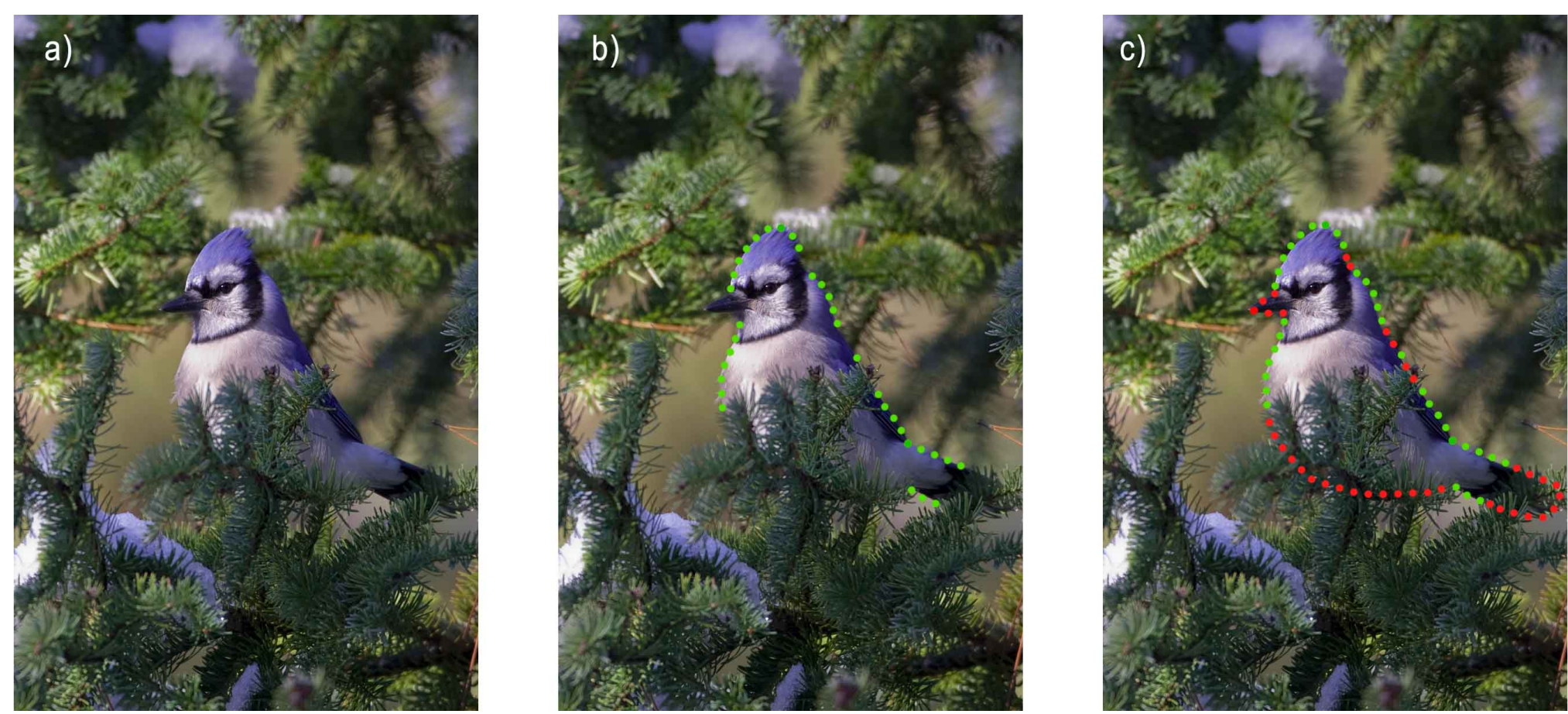

Figure 2.3 Contour completion: from detected edges to global shape. (a) Natural viewing condition of a blue jay (Cyanocitta cristata), with portions of its edge not detected due to low contrast with background (i.e. its beak) or occlusion from foreground branches (i.e. its underbelly), (b) Contour integration (green dots) groups detected edges into a partially complete boundary, (c) Contour completion (red dots) fills in the missing boundary information (due to low contrast animal-background boundaries and occlusion) to form a closed shape estimation of the animal. 
The final stage of object recognition is to utilize shape information for object identification. Since Thayer (1909), a body of evidence has emerged suggesting that shape is of key importance for object recognition (Delvenne and Dent, 2008, Liebe et al., 2009, Soto and Wasserman, 2012), particularly for animal recognition by humans (LloydJones et al., 2010, Lloyd-Jones and Luckhurst, 2002, Elder and Velisavljevic, 2009). Furthermore, recognition of shape can contribute to detectability independently of an object's colour (Ings et al., 2012, Brown et al., 1992, Carlile et al., 2006, Schluessel et al., 2012, Lehrer and Campan, 2004). This concept has implications for the hypothesis that disruptive coloration can achieve camouflage independent of background matching; for instance, a small loss of background matching could be tolerated if the change derives a larger benefit from degrading shape recognition.

There is thus considerable evidence, reviewed above, that the visual systems of humans and non-human vertebrates share properties of shape perception (such as perceptual grouping). For instance, even though mammals and birds have very different brain anatomies (Hodos, 1993), shape recognition is not just present in both these taxa (Logothetis et al., 1995); (Peissig et al., 2005, Nielsen and Rainer, 2007), but also operates in a similar manner (Nielsen and Rainer, 2007).

\section{(b) Visual attention}

Object recognition in real-world scenes is a cognitively demanding task (Dukas, 2004), and visual attention dictates when each part of the visual field is processed. Visual attention involves prioritizing portions of the scene for inspection, moving the eye's 
focus to the region of interest and then based on the new information acquired return to the task of prioritizing the scenes for inspection. This part-by-part approach to detailed inspection of the scene is a solution to avoiding the visual and cognitive overload that would occur if the whole scene were to be processed concurrently (Tsotsos, 1990). Two broad types of information determine which portions, and in what order, of the surrounding environment should be visually processed; namely, bottom-up and top-down factors.

Saliency is defined as the bottom-up distinctiveness of an object, relative to its surroundings (i.e. a colour background-matching animal's saliency is relative to the visual elements of its environment (Fecteau and Munoz, 2006)). Importantly, saliency contributes to visual attention independently of task. Some properties that affect an object's saliency are colour, orientation, size and movement (Wolfe, 1998). ${ }^{4}$

To predict visual attention, the saliency of objects in a scene is combined with the top-down relevance of the information to the current task: together, this explains how visual attention prioritizes information (Fecteau and Munoz, 2006). In animals, this computational task is achieved by many specialized regions of the brain (O'Connor et al., 2002), which mediate visual attention through guiding eye movements to regions of interest (Itti and Koch, 2001).

\footnotetext{
${ }^{4}$ Backgrounds with many competing salient visual elements are more cognitively demanding to search. Due to the limits of attention, there can arise a trade-off between foraging in these visually-challenging environments and antipredator vigilance behaviours (Dukas 2004).
} 
When a predator's visual attention is focused on a disruptively coloured prey and that prey succeed in being seen but not recognized, it is doubly advantageous to the prey. It has avoided being attacked and secondly this false-positive leads the predator's visual attention to be less likely to return (Geilser 2000) and inspect that patch (Klein and MacInnes, 1999).

\subsubsection{Visual mechanisms}

Exploiting shape perception underpins disruptive coloration; so when testing the function of disruption, it is important to determine how different visual mechanisms influence the role of shape in object recognition. One important feature of the vertebrate visual system is how foveal and peripheral visions vary in their ability to perceive shape information. Foveal vision allows high-quality shape perception, whereas peripheral vision is pre-attentive and limited in its shape perception (Palmer 1999). Disruptive coloration should improve camouflage when shape information is valuable for object recognition (i.e. in foveal vision), because distorting this information will contribute to greater reduction in recognition.

\section{(a) Foveal vision}

Eye movement serves a critical role in the process of visual attention and is a behaviour that provides insight into how object recognition occurs. Spatial resolution of the eye varies owing to the different densities of rods and cones across the surface of the retina, which are high at its centre and then decrease towards the periphery (a phenomenon called retinal eccentricity (Palmer, 1999)). Consequently, there is greater 
acuity in the centre of the visual field (foveal vision) and diminished sensitivity towards the margins (peripheral vision). Several behavioural ecology questions are now measuring attention using the indirect approach of measuring head position (FernándezJuricic et al., 2011) and more directly through recording eye-tracking (Yorzinski et al., 2013, Levy et al., 2013)

High-speed eye movements direct foveal vision toward target locations that are determined by visual attention (Palmer et al., 2000). The periods of time when the eye jumps from point-to-point are called saccades, and during these periods no new visual information is gathered (Matin, 1974). Following saccades, there is a fixation period of time during which the eye remains somewhat still, as high-acuity foveal vision gathers localized information. Interestingly, these rapid visual inspections are not instantly forgotten, thereby allowing content-rich information to be incrementally gathered, first for the portions of the scene of highest priority, and then building a wider coverage. For object recognition to occur, it is generally assumed that foveal vision is required because it has superior shape perception (Palmeri and Gauthier, 2004).

Because shape information plays a prominent role in identifying objects during foveal vision, it would seem likely that disruptive coloration should affect foveal eye movements to be effective. By simultaneously comparing eye movements, visual attention to an object, and behavioural outcomes (e.g. identifying target objects), one can measure the time taken to recognize an object and the number of failed recognition attempts (false-positives). Disruptive coloration is predicted to break up the outlines or 
shapes of animals, thereby impairing their recognition. This leads to a prediction that eyes will fixate on disruptive targets for longer periods of time than on non-disruptive targets, as information on their shape is degraded and thus it takes longer for the receiver to recognize them, which is directly tested in Chapter 6.

\section{(b) Peripheral vision}

Although object recognition is predominately the domain of foveal vision, peripheral vision is also involved. Only a very small portion of the visual field consists of foveal vision (e.g. in humans, foveal vision consists of only $2^{\circ}$ of the $120^{\circ}$ field of view (Palmer et al., 2000)). Outside of foveal vision, acuity and colour sensitivity are diminished. This poor processing of spatial frequencies ${ }^{5}$ by peripheral vision (Rentschler and Treutwein, 1985, Bennett and Banks, 1987) leads to degraded contour integration (Hess and Dakin, 1997) that reduces shape perception performance (e.g. straight boundaries are easier for the peripheral visual to aggregate than curvilinear boundaries (Hess and Dakin, 1997, Hess and Dakin, 1999)). Traditionally, the consensus has been that the high-level visual processing required for object recognition depends on eye movements to provide an attentional 'spotlight' (Liversedge and Findlay, 2000), with peripheral vision not able to recognize objects. However, this assumption of peripheral vision's lack of participation in object recognition processing has received criticism. Several studies have shown that the metaphor of the foveal attentional spotlight (Posner, 1980) fails to describe the gradual fall-off of object recognition processing (Hoffman and

\footnotetext{
${ }^{5}$ Spatial frequencies are a common metric used to describe visual acuity per unit distance (e.g. in visual perception this refers to the resolution per degree of visual angle). For instance, the capacity of a visual system's spatial frequency is tested using a piece of paper across which there are printed black lines that go from thick to very thin displayed at a set distance; the point the visual system stops being able to distinguish between the black and white line is the visual system's maximum resolution. Spatial frequencies also denote the information about an object's visual appearance. High spatial frequencies convey fine details of textures, whereas low spatial frequencies convey edge and global shape information.
} 
Nelson, 1981, Laberge, 1983, Eriksen and Yeh, 1985, Yao et al., 2011), as object recognition in humans continues beyond the $2^{\circ}$ foveal vision region. Yao et al. (2011) measured the field of attention wherein object recognition occurs and found that, whilst object recognition is highly accurate in foveal vision, a distance ten-fold beyond this field can contribute to recognition but with diminished accuracy (Yao et al., 2011). This finding suggests that pre-attentive peripheral vision contributes to object recognition, but perhaps only when objects are highly salient, such as in being very large (Thorpe et al., 2001) or distinctly coloured (Chang et al., 2012). Thus, peripheral vision may play a role in the recognition of disruptively-coloured objects. However, due to a combination of poor shape processing, which is further diminished for background matching targets (as they poses low contrast edges), the effects of disruption on shape recognition should be much more pronounced in foveal vision.

\subsection{Synthesis}

This chapter introduced the literature on disruptive coloration and provided a framework for the proximate mechanisms involved in object recognition (a process that underpins disruptive function). Whilst there has been a recent resurgence of interest in camouflage and disruptive coloration, and acceptance that explanation of animal coloration requires functional description (Stevens and Merilaita, 2009), few studies have tested how disruptive coloration functions. The following data chapters of this thesis undertake needed functional tests of disruptive coloration and of camouflage more broadly. 


\section{Chapter 3: Behaviourally mediated crypsis in two nocturnal moths with contrasting appearance}

"Moths select resting substrates that match the reflectance of their forewings and adopt species-typical body orientations on the substrate by which they appear to align their disruptive markings..." (Pietrewicz and Kamil, 1977)

\subsection{Abstract}

The natural resting orientations of several species of nocturnal moth on tree trunks were recorded over a three-month period in eastern Ontario, Canada. Moths from certain genera exhibited resting orientation distributions that differed significantly from random, whereas others did not. In particular, Catocala spp. collectively tended to orient vertically, whereas subfamily Larentiinae representatives showed a variety of orientations that did not differ significantly from random. To understand why different moth species adopted different orientations, I presented human subjects with a computer-based detection task of finding and 'attacking' Catocala cerogama and Euphyia intermediata target images at different orientations when superimposed on images of sugar maple (Acer saccharum) trees. For both C. cerogama and E. intermediata, orientation had a significant effect on survivorship, although the effect was more pronounced in $C$. cerogama. When the tree background images were flipped horizontally the optimal orientation changed accordingly, indicating that the detection rates were dependent on the interaction between certain directional appearance features of the moth and its background. Collectively, my results suggest that the contrasting wing patterns of the moths are involved in background matching, and that the moths are able to improve their crypsis through appropriate behavioural orientation.

Permission to reproduce this published journal article was granted by The Royal Society: WEBSTER, R.J., CALLAHAN, A., GODIN, J-G.J \& SHERRATT, T.N. 2009. Behaviourally mediated crypsis in two nocturnal moths with contrasting appearance. Phil. Trans. R. Soc. B., 364, 503-510. 


\subsection{Introduction}

The best-known interrelated mechanisms through which coloration can act to reduce the detection rates of potential prey are background matching and disruptive coloration (Thayer, 1909, Cott, 1940, Kingsland, 1978, Ruxton et al., 2004b, Wilkinson and Sherratt, 2008, Stevens and Merilaita, 2009). With background matching, objects are difficult to detect simply due to their similarity to the background. Conversely, the striking/high-contrast markings involved in disruptive coloration create 'the appearance of false edges and boundaries and hinders the detection or recognition of an object's, or part of an object's, true outline and shape' (Stevens and Merilaita, 2009).

In recent years, much effort has been directed at understanding the disruptive coloration principle and there is now considerable evidence that disruptive markings in prey items serve to reduce their detectability by predators (Merilaita, 1998, Cuthill et al., 2005, Merilaita and Lind, 2005, Stobbe and Schaefer, 2008, Fraser et al., 2007, Kelman et al., 2007, Stevens et al., 2006a). Many of these studies carefully controlled for background matching by altering only the position of the high-contrast elements of the objects of interest. However, in asking questions about disruptive coloration while directly controlling for background matching, one might get the impression that the two principles are readily separable (Stevens, 2007). While disruptive coloration can operate successfully independent of background matching (Schaefer and Stobbe, 2006), it seems that they are to an extent interdependent (Fraser et al., 2007, Stevens, 2007), a stance adopted in Cott's (1940) landmark text on animal coloration: ' $\ldots$ the effect of disruptive pattern is greatly strengthened when some of its components closely match the 
background'. Here, I extend the research by demonstrating that contrasting wing markings (with seemingly, yet so far unproven, disruptive functions) within two groups of nocturnal moths can act to facilitate background matching. Moreover, I show that the effectiveness of the camouflage in these moths is enhanced by selection of appropriate resting orientations.

Much previous work has been done on artificial (Pietrewicz and Kamil, 1977, Bond and Kamil, 2002, Bond and Kamil, 2006, Fraser et al., 2007) and natural (Sargent, 1969b, Sargent, 1969c, Sargent, 1969a, Sargent, 1969d, Sargent, 1968, Endler, 1984, Moss et al., 2006) moth crypsis, including several classical studies that have revealed the importance of orientation on crypsis. In particular, Pietrewicz \& Kamil (1977) explored the effect of body orientation in Catocala spp. by presenting blue jays (Cyanocitta cristata) with slides of moths in different orientations on trees. They showed that both the moth orientation and tree species combined to influence the birds' prey detection rate, such that orientation affected detectability but only on some tree species. While this pioneering experiment was an important step towards establishing direct evidence that behavioural orientation influences crypsis, there were some limitations, including inevitable low sample size of predators and the fact that only three levels of orientation were explored (up, down and right). Most importantly, although the authors described the high-contrast markings in the Catocala species they investigated (which they referred to as faint or prominent disruptive patterns), the underlying reasons for the orientation effects they observed were not further investigated. 
In the present study, I investigated the importance of orientation in reducing detectability and conducted tests to elucidate the underlying mechanisms involved. First, I set out to determine whether two groups of naturally occurring moth species orient nonrandomly on tree trunks in the field, and whether there was any between-group variation. I then used a computer-based system of humans 'foraging' for images of the moths against images of trees to test whether moth orientation influenced survivorship in my system, and, if so, whether the results were consistent with the field data on the natural orientations of the moth species concerned. Finally, to test whether the moth orientation effect could be explained by the moths' alignment to trees' patterns, I horizontally rotated the same tree images that were presented, and asked whether the optimal orientations of the moth species were concomitantly altered.

\subsection{Methods}

\subsubsection{Field survey of moths' body orientation}

Moths were intensively searched for in two mixed deciduous forests near Ottawa, Canada, between 21 June and 8 August 2006. The forests were Stony Swamp Conservation Area $\left(45^{\circ}, 17^{\prime}, 58.29^{\prime \prime} \mathrm{N} ; 7^{\circ}, 49^{\prime}, 11.06^{\prime \prime} \mathrm{W}\right)$ and Monk Woods Environment Park $\left(45^{\circ}, 20^{\prime}, 23.86^{\prime \prime} \mathrm{N} ; 7^{\circ}, 55^{\prime}, 55.84^{\prime}\right.$ ' W), with common tree species being basswood (Tilia americana), bitternut hickory (Carya cordiformis), bur oak (Quercus macrocarpa), ironwood (Ostrya virginiana), red maple (Acer rubrum), red oak (Quercus rubra), sugar maple (Acer saccharum), white ash (Fraxinus americana) and white birch (Betula papyrifera). My protocol involved taking transects through the field sites and any tree, of girth greater than $10 \mathrm{~cm}$, was assessed for the presence of resting 
moths. This assessment was a two-stage process. First, from several metres away, the tree was visually scanned. Then, the tree was approached and a tactile search was used. This touching of the tree ensured that no moths on that tree had been overlooked. If a moth had been missed, then 'tapping' the tree was intended to frighten the moth out of hiding. While in such cases the data on moth orientation were lost, it provided a good fail safe, ensuring that the most cryptic moths on tree trunks were not being missed. Clearly, this method only provides an assessment of moths' presence on lower sections (the first $3 \mathrm{~m}$ ) of tree trunks and no attempt was made to search leaf litter or higher branches.

On locating a moth in its natural resting position, I recorded the moth species, host tree species and time of day. A photograph of the moth in its natural position was taken using a Canon PowerShot Pro1 from roughly $30 \mathrm{~cm}$ away. The camera's lens was then set to its widest zoom to ensure that the edges of the tree were included in the frame. These photographs were later used to extract orientations of moths, relative to that of the tree, using ImageJ. After these in situ recordings were completed, I captured the moth using either a net or a jar and stored it for later confirmation of species identity.

\subsubsection{Human predator system: testing the effect of orientation on crypsis}

Between July and September 2007, approximately 20 nights were spent light trapping to collect new moth specimens, including those species that were most commonly found in the field from the previous year. This collection effort yielded nine good-condition specimens of Catocala cerogama (more Euphyia intermediata were caught but only nine were used for this experiment, matching the number of C. cerogama 
specimens used). Specimens were caught at night, collected in pill jars, refrigerated until the morning and killed using an ethanol-laced jar. Once dead, the specimens were mounted together on brown card in a natural resting position (L. Scott 2007, personal communication). C. cerogama has complex contrasting markings, in the form of wavy lines with no clear directionality (Fig. 3.1). As well as its concealment coloration, $C$. cerogama has conspicuous markings on its hind wings, but they are masked by cryptic forewings when at rest. Catocala species are also known for their polymorphism (Bond \& Kamil 2002), but the colour pattern differences among the individual moths used in the experiment were relatively subtle. The specimens of E. intermediata all had high-contrast markings, forming a band perpendicular to its body axis (figure 1). During the 2006 field season, both of these species were commonly found on sugar maple (A. saccharum) trees, but they showed no significant preference in choosing this tree species over other trees (Callahan, 2007).

The nine specimens of each moth species (C. cerogama and E. intermediata), all mounted on brown card, were photographed in the field against each of nine sugar maples. All photographs were taken on overcast days in September 2007. Trees were photographed with and without the moth specimens in quick succession to ensure identical lighting conditions: of these photographs, the ones without the moths were used as background images and ones with, were merely used to excise moths. Moths and trees were photographed using a Canon EOS D60 with an EF 24-70 mm f/2.8 L USM lens mounted on a tripod with a distance of $120 \mathrm{~cm}$ between camera's sensor and the tree. The zoom was set to $55 \mathrm{~mm}$ (equivalent to the human eyes' diagonal field of view (Ray, 
2002). Photographs were recorded in RAW to enable the colour temperature to be selected post-capture. By photographing on dull overcast days, I minimized non-uniform illumination of the tree caused by dappled sunlight; such variation in lighting conditions within the image of the tree are unwanted because they will contribute to an artificial enhancement of the moths' conspicuousness, due to the excised moth no longer matching the illumination of its background.

Each tree background was matched with an image of a unique C. cerogama and E. intermediata specimen, excised from the mounted sample of moths photographed under the same conditions as the tree was photographed. The uniform brown card region around each moth was selected using the Adobe Photoshop magic wand tool, with a low tolerance set to between 5 and 20 RGB colour value deviation, between neighbouring pixels. This selected area was deleted and then the edges around the moth were manually cleaned using the Adobe Photoshop eraser to remove shadows, leaving just the moth. Moth targets were saved as .PNG files with transparent backgrounds. This process was repeated for two unique specimens (one per species) for each of the nine trees. Since $C$. cerogama is approximately six times the surface area of E. intermediata, I enlarged $E$. intermediata targets and their respective backgrounds to render this second target species more comparable in size. This resulted in forming a new set of 'zoomed in' background images, derived from the original background image set. Irrespective of the species, the dimensions of the background tree were 600 pixels wide $\times 900$ pixels high (and the reverse for horizontally rotated trees) while the moth target images (transparent outside 
the actual image of the moth) were placed to fill a square area of 75 pixels wide $\times 75$ pixels height.

I developed a Microsoft Visual Basic (Visual Basic 2008) application to present images of moth target super-imposed on tree backgrounds and to quantify the elapsed time that human subjects took to detect moths (assuming it was detected at all). For each human subject, a set of 90 tree images were presented on a computer monitor. In doing so, the nine tree backgrounds I had photographed were each presented 10 times: for eight of these presentations the moth target was randomly positioned in the image and set as to one of eight orientations $\left(0,45,90,135,180,225,270\right.$ and $315^{\circ}$ from vertical $)$, and twice with no moths present. Each image presented to human subjects was a unique pairing of a moth's orientation against its appropriate background — no subject was presented with a given moth in a given orientation more than once, so as to avoid pseudo-replication. The human subjects were presented a maximum of one moth per background (but one-fifth of the time there was no moth target). Moth orientation, as well as presence/absence, was randomized as to the order they appeared. Tree background images were presented in sequence, cycling through all backgrounds before repeating the same backgrounds again: this ensured that the same tree background image was never presented one after the other to invoke change blindness, so that the moth superimposed on the same tree image are not instantly recognizable from short-term visual memory (Rensink et al., 1997). This order was changed between human subjects. Overall, therefore, the same moth image was presented against the same tree image in eight different orientations and I presented a total of nine completely different moth-tree pairs (hereafter referred to simply as tree) in 
the same way ( 8 orientations $\times 9$ trees $=72$ moths in total). For each human observer 9 blank trials (catch trials ${ }^{6}$ ) were present randomly throughout the experiment.

One of four foraging environments was presented to each human subject (two species, by two background orientations). For each foraging environment (i.e. $C$. cerogama on vertical background, C. cerogama on horizontal (flipped) background, E. intermediata on vertical background and E. intermediata on horizontal (flipped) background), I collected data from 24 human subjects (96 different subjects in total), thereby ensuring complete orthogonality in design.

Subjects participated in this human predator system experiment at the Carleton University Maxwell MacOdrum Library. Two computer terminals were set up with the Visual Basic application, viewed using 1900 Stealth Computer Corporation LCD monitors with a screen resolution of $1024 \times 768$ pixels. Cardboard screens were erected to minimize disturbance to participants and to reduce the effect of ambient light.

Participants were given no indication of the purpose of the experiment, only that it was a foraging exercise and they were only allowed to participate once. The first window presented a trial screen, with a moth in all eight different orientations simultaneously (in random, yet non- overlapping positions) and the subject was asked to find all the moths on the screen — this acted as a short training period for the naive subjects. The trial screen resembled the screen for the real experiment and the researcher orally explained which

\footnotetext{
${ }^{6}$ Catch trials are a psychophysical definition given blank trial used for signal detection theory calculations. This would be an interested way to extend this work, but more catch trial would be needed.
} 
buttons to press. The time taken to attack moths (in milliseconds) from the first presentation of the moth was recorded automatically.

\subsubsection{Statistical analysis}

I quantified the in situ resting body orientation of free-ranging moths in the field from photographs using ImageJ. The resting site angular distributions for each species were tested using Rayleigh's test for angular distributions (Zar, 1999), with the null hypothesis that moth resting orientations were uniformly distributed.

To examine the effect of moth orientation (eight levels) on moth 'survivorship', I fitted general linear models (GLMs) to the data. All statistical tests were carried out using SPSS v.15. Throughout the analyses, moth orientation was expressed in one of two complementary ways: either absolute (so that the north-south axis, for example, is always upwards-downwards on the monitor) or relative to the trees rotation (such that northsouth axis is always along the trunk of the tree: this measure is therefore sensitive to the tree background rotation). To avoid complex interactions in the fitted model, a separate GLM was fitted to the data for each moth species. For the first fit of the GLM, the dependent variable was the proportion of the 24 human subjects (arcsine transformed) that failed to detect a moth when it was presented in a particular orientation on a particular tree, and the tree was presented vertically or horizontally. Moth orientation (absolute or relative) and tree rotation were treated as fixed factors, while tree (representing a subset of possible factor levels) was treated as a random factor. All pairwise interactions were included, but higher order interactions were necessarily omitted. To complement this analysis, I fitted another GLM, with the time taken to detect 
those moths that were attacked the dependent variable (square root, log transformed to ensure normality and homogeneity of variance). Here, moth orientation was treated as a fixed factor, while tree and human subject were treated as random factors. By including a human subject effect, data from the two tree rotations were necessarily considered separately (since human subjects only participated in one of the four experiments).

\subsection{Results}

\subsubsection{Field survey of moths' body orientation}

Fifteen specimens of Catocala spp. (comprising C. cara, C. cerogama, C. ilia, C. semirelicta, C. subnata and C. unijuga) and 11 specimens of the family Larentiinae (comprising Epirrhoe alternata, E. intermediata, Xanthorhoe labradorensis) were found in natural resting positions. Catocala spp. exhibited a highly significant preference for head-up/head-down orientation (Fig. 3.1) between 60 and $105^{\circ}$ and 235 and $272^{\circ}(Z=$ $11.2, \mathrm{n}=15, p<0.001)$. However, I could not reject the null hypothesis that Larentiinae representatives were orientated uniformly (Fig 3.1) on tree trunks $(\mathrm{Z}=1.2, \mathrm{n}=11, p>$ $0.05)$. 
(a)
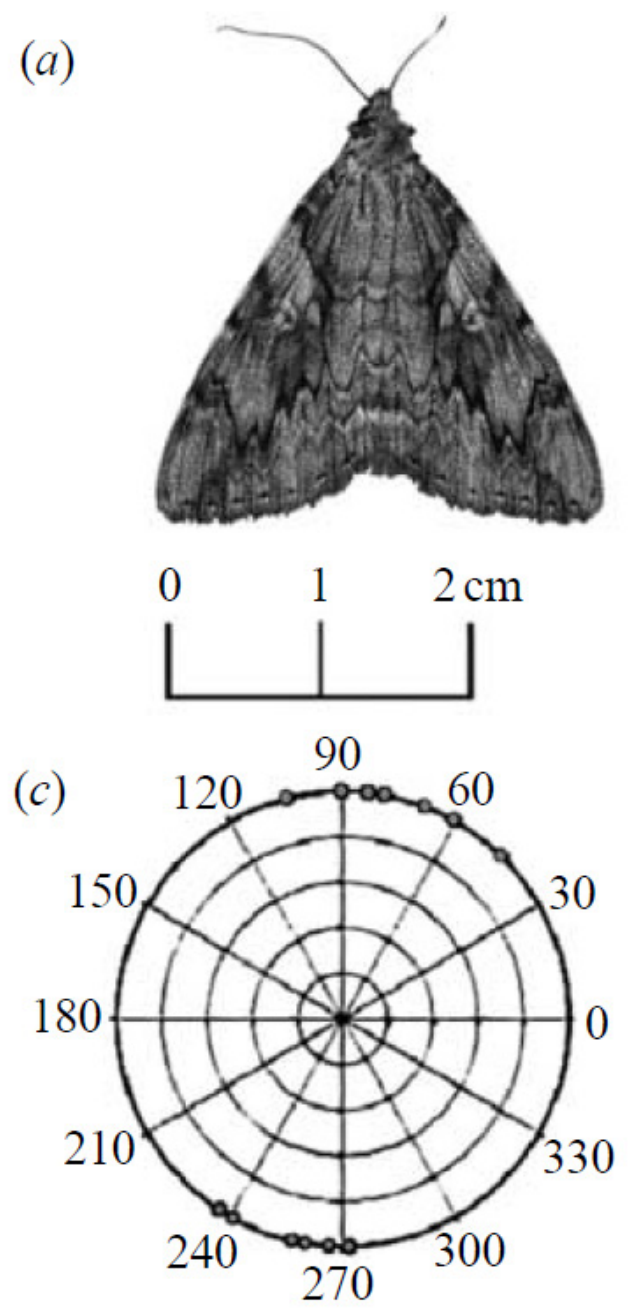

(b)
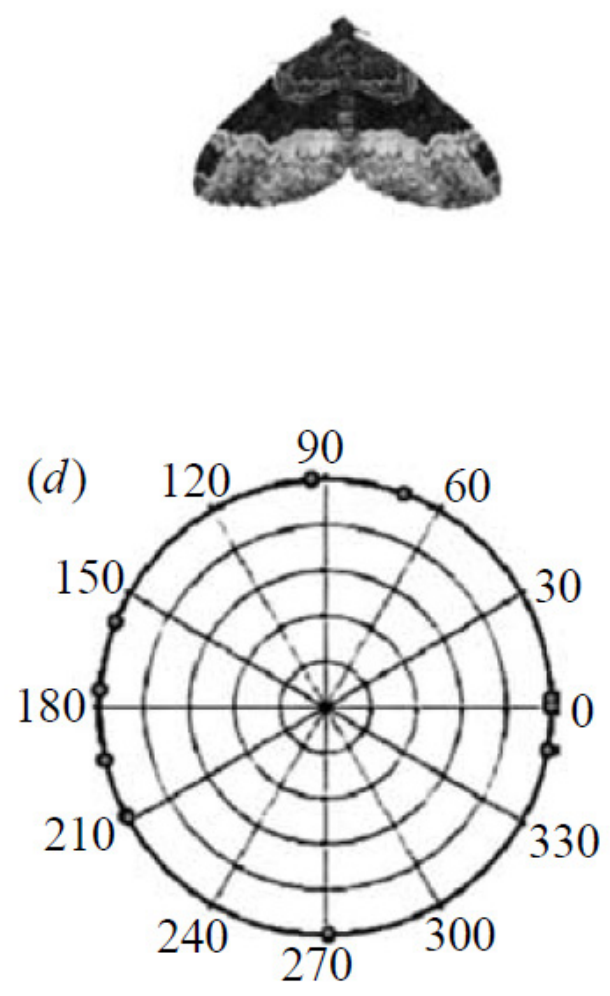

Figure 3.1: Photographs of the study species (a) C. cerogama and (b) E. intermediata and the angular distributions of individual (c) Catocala spp. and (d) Larentiinae representatives found on trees in the field. Black circles mark the position of the head, relative to the orientation of the tree $\left(90^{\circ}\right.$ at vertical). Angular distributions are clearly non-uniform for Catocala spp., yet relatively uniform for Larentiinae representatives. 


\subsubsection{Human predator system: testing the effect of orientation on crypsis}

On fitting a GLM to the arcsine transformed proportion of moths missed per person for each species, the main effects of tree and tree rotation were significant for both species (Table 3.1). There was no significant effect of absolute moth orientation, but the interaction term of absolute moth orientation $\times$ tree rotation was significant for both species (Table 3.1; Fig. 3.2). When relative moth orientation was used instead of absolute moth orientation in the same model, the relative moth orientation became significant while the moth orientation $\times$ tree rotation effect became non-significant (Appendix Table C.1). This suggests that it is the orientation of the moth relative to the tree that is primarily responsible in influencing detectability (not the absolute orientation of the moth).

For the GLM where detection time was set as its response variable, the main effects of tree were significant for both species, but moth orientation was only significant for C. cerogama (Table 3.2; Fig. 3.3), indicating that any orientation effect on attack time, if present, was less marked for E. intermediata. Indeed, the significant human subject $\times$ moth orientation interaction suggests that orientation had an effect on detection time in E. intermediata, but that this effect was rather subject specific. Nevertheless, changes in the mean detection paralleled the proportion of moths missed in their different orientations (Fig. 3.2 and Fig. 3.3). For the GLM where detection time was set as its response variable, the main effects of tree were significant for both species, but moth orientation was only significant for C. cerogama (table 2; figure 3), indicating that any orientation effect on attack time, if present, was less marked for E. intermediata. Indeed, 
the significant human subject $\times$ moth orientation interaction suggests that orientation had an effect on detection time in E. intermediata, but that this effect was rather subject specific. Nevertheless, changes in the mean detection time of those moths that were eventually attacked roughly paralleled the proportion of moths missed in their different orientations (Fig. 3.2 and 3.3). 


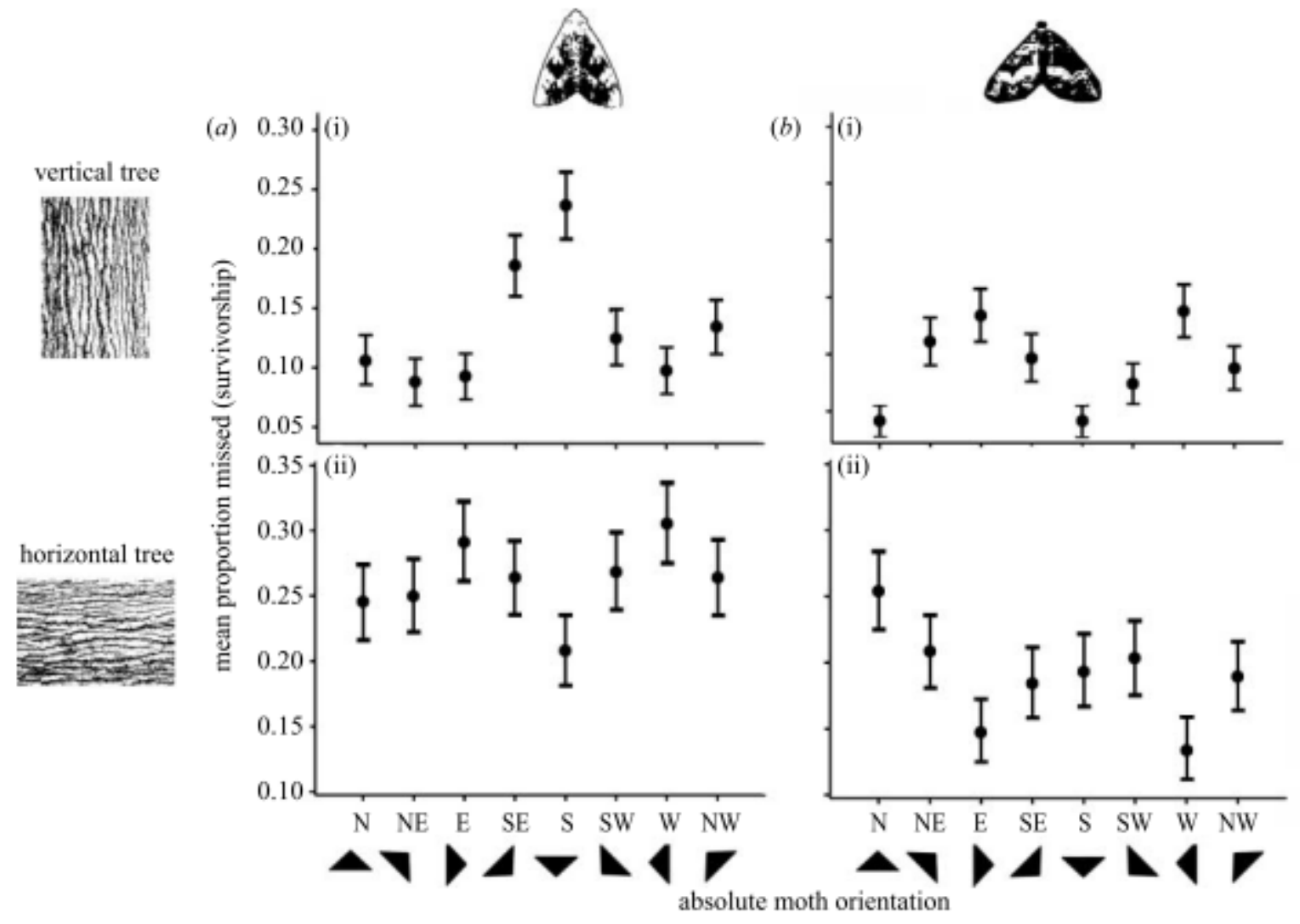

Figure 3.2: Mean ( \pm 1 S.E.) proportion of moth targets missed $((a) C$. cerogama and $(b)$ E. intermediata) on (i) vertically and (ii) horizontally rotated trees according to absolute moth orientation. Images of moth species (not to scale) have been thresholded to illustrate their high-contrast marking parallel and perpendicular to body axis. 
Table 3.1: GLMs of arcsine-transformed overall mean proportion missed (survivorship) per human subject for each moth species with three main effects (absolute moth orientation, tree rotation and tree) and all pairwise interactions. Test statistics for the GLM are represnted: FS (d.f.) significance $\left(* * * p<0.001,{ }^{* *} p<0.005,{ }^{*} p<0.05, p>0.05=\right.$ n.s.). All factors in the GLM are fixed, except for tree which is a random factor.

\begin{tabular}{llr}
\hline GLM factors and interactions & C. cerogama & E. intermediata \\
\hline absolute moth orientation & $1.95(7,56) \mathrm{n} . \mathrm{s}$. & $1.17(7,56) \mathrm{n} . \mathrm{s.}$ \\
tree rotation & $11.66(1,8)^{*}$ & $5.64(1,8)^{*}$ \\
tree & $26.26(8,2.69)^{*}$ & $30.51(8,6.99)^{* * *}$ \\
absolute moth orientation $\times$ tree rotation & $4.50(7,56)^{* * *}$ & $3.47(7,56)^{* *}$ \\
absolute moth orientation $\times$ tree & $0.70(56,56) \mathrm{n} . \mathrm{s.}$ & $0.66(56,56) \mathrm{n} . \mathrm{s}$. \\
tree $\times$ tree rotation & $0.87(8,56) \mathrm{n} . \mathrm{s}$. & $5.4 .3(8,56)^{* * *}$ \\
\hline
\end{tabular}




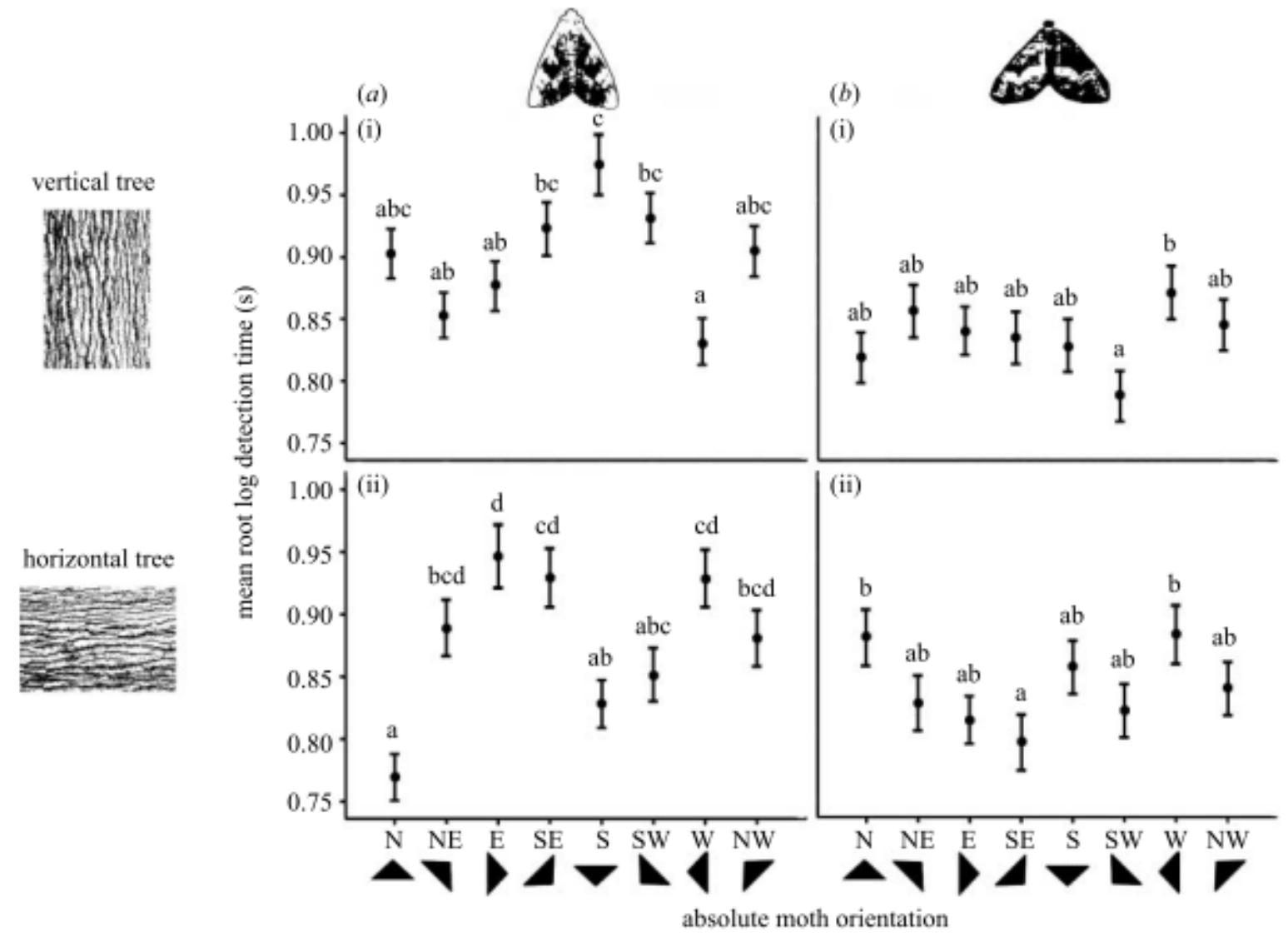

Figure 3.3: Mean ( \pm S.E.) detection time for moth targets $(a)$ C. cerogama and $(b) E$. intermediata) on (i) vertically and (ii) horizontally rotated trees according to absolute moth orientation. Images of moth species (not to scale) have been thresholded to illustrate their high-contrast markings. The letters assigned to each error bar are generated from Tukey's post hoc analysis and represent statistically homogeneous subsets at $\alpha=0.05$ for each orientation. 
Table 3.2: GLMs of square root, log-transformed detection time of each moth attacked, for each species and tree rotation with three main effects (absolute moth orientation, human subject and tree) and all pairwise interactions. Test statistics reported for the GLM are:

FS (d.f.), significance $(* * * p<0.001, * * p<0.005, * p<0.05, p>0.05=$ n.s. $)$.

\begin{tabular}{llll}
\hline & GLM factors and interactions & C. cerogama & E. intermediata \\
\hline vertical & absolute moth orientation & $5.10(7,45.9)^{* * *}$ & $0.98(7,73.2) \mathrm{n} . \mathrm{s}$. \\
& tree & $17.41(8,65.4)^{* * *}$ & $\begin{array}{l}10.00(8,105.06)^{* * *} \\
3.73(23,199.5)^{* * *} \\
\end{array}$ \\
& human subject & $4.32(23,104.5)^{* * *}$ & $1.12(56,846) \mathrm{n} . \mathrm{s}$. \\
& absolute moth orientation $\times$ tree & $1.37(56,1054)^{*}$ & $1.26(161,846)^{*}$ \\
& absolute moth orientation $\times$ human subject & $0.94(161,1054) \mathrm{n} . \mathrm{s}$. & $1.77(172,42.1)^{* * *}$ \\
tree $\times$ human subject & $1.25(183,1054)^{*}$ & $1.99(7,74.81) \mathrm{n} . \mathrm{s} .(p=0.068)$ \\
& absolute moth orientation & $7.02(7,45.0)^{* * *}$ & $28.53(8,42.16)^{* * *}$ \\
& tree & $20.81(8,67.6)^{* * *}$ & $4.03(23,113.91)^{* * *}$ \\
& human subject & $5.01(23,111.5)^{* * *}$ & $0.95(56,954) \mathrm{n} . \mathrm{s}$. \\
& absolute moth orientation $\times$ tree & $1.45(56,1124)^{*}$ & $0.95(161,954) \mathrm{n} . \mathrm{s}$. \\
& absolute moth orientation $\times$ human subject & $1.02(161,1124) \mathrm{n} . \mathrm{s}$. & $0.97(177,954) \mathrm{n} . \mathrm{s}$. \\
\hline
\end{tabular}




\subsection{Discussion}

Since Kettlewell's (1958) classical experiments on background selection by melanic forms of Biston betularia, there has been much debate regarding the natural resting location of moths on trees (Sargent, 1966). I do not discount the possibility that the moth species in this study use tree branches and even leaf litter for concealment. However, even if only a small proportion of moths naturally choose tree trunks to rest on, I suspect it is these moths that have most to gain from appropriate concealment coloration and behavioural alignment. Moreover, my questions were necessarily limited to whether those moths found on tree trunks exhibited a non-uniform resting orientation and whether this choice of resting orientation could be understood on the basis of reduced detectability.

It has long been known that many moth species exhibit non-random resting orientations on both artificial (Sargent, 1966, Sargent, 1968, Sargent and Keiper, 1969) and natural (Endler, 1984) substrates. Such orientation behavior has been assumed to enhance moth crypsis, although the underlying mechanisms remain poorly understood. In this experiment, I extended previous work done on the resting orientations of nocturnal moths on trees by manipulating the rotation of the background relative to that of the moth. My primary aim here was to test whether the moth orientation effect was a product of some form of interaction between directional aspects of the appearance of the moth and its background. 
I found that moth orientation had a significant effect on crypsis and that the optimal orientation depended on the background's rotation. Thus, moth orientation was shown to have a significant effect on survivorship in the combined dataset of vertical and rotated tree images, showing up as an interaction between absolute moth orientation $\times$ tree rotation (Table 3.1) and in the main effect of relative moth orientation (Appendix Table C.1). Collectively, these results indicate that the maximally cryptic moth orientations are influenced in some important way by the rotation of the tree. For example, south orientated C. cerogama had the highest survivorship on vertical trees but the lowest survivorship on horizontal trees. Likewise, west and east orientated $E$. intermediata had the highest survivorship on vertical trees but the lowest survivorship on horizontal trees (Fig. 3.2). One might argue that the effects of tree rotation on the proportion of moths missed (and the mean detection time of moths attacked) arose because I used a different group of humans for vertical and horizontally rotated trees. However, I feel it highly unlikely that the roughly perpendicular switch in optimal orientations arose for any other reason than the perpendicular switch in tree rotation.

A key feature of sugar maple bark is its prominent furrows, creating high-contrast patterning running up and down the tree. By presenting the tree images vertically (natural) and horizontally (artificially rotated), the directionality of the trees' prominent patterns was altered. Given the corresponding changes in optimal orientation of moths with tree rotation, it seems likely that the different markings on the moths (and/or their shape) somehow match those of the bark. My interpretation is further supported when I note that the two moth species investigated have contrasting patterns and shapes (with $E$. 
intermediata exhibiting clear markings perpendicular to the axis of its body and $C$. cerogama exhibiting a more complex pattern) and also exhibit different optimal orientations. That said, certain aspects of these data are not readily understood. For example, south orientated C. cerogama on vertical trees appear to have higher survivorship than north orientated moths under the same conditions. This outcome seemingly arises as a product of some subtle interaction between moth body pattern (or even moth shape) and tree orientation, although it is difficult to identify the source of this interaction. Pietrewicz \& Kamil (1977) likewise found differences in the detectability of south and north orientated Catocala, the precise result varying with Catocala species.

It is possible that selective forces other than crypsis act on moth orientation in the field; for instance, orientating down for ease of escape from predators. However, reassuringly my field data on C. cerogama's non-uniform resting orientation correspond to the orientations that have been shown to maximize survivorship in the human predator system. For this case at least, it would seem selective pressures to enhance crypsis have influenced C. cerogama's resting orientation. For the Larentiinae representatives, the null hypothesis that natural resting orientation is uniform could not be rejected. This result could be a false negative (indeed $75 \%$ of all records of this moth were within $\pm 30^{\circ}$ of the horizontal, whereas only $25 \%$ of records were within $\pm 30^{\circ}$ of the vertical). However, I note that moth field resting orientation was necessarily combined between tree species and it is possible that Larentiinae representatives orient in different fashions on different tree species. Likewise, while Larentiinae representatives have highly similar colour patterning, E. alternata, E. intermediata and X. labradorensis could conceivably show 
heterogeneous orientation behaviour. A final, unlikely, alternative hypothesis is that Larentiinae representatives' high-contrast markings allow their (potentially) disruptive camouflage to function independent of orientation.

Different tree-moth combinations seem to differentially affect the degree to which moth orientation influences survivorship. The effect of tree specimen on moth survivorship under different orientations is apparent in Appendix Fig. C.1, which indicates that survivorship differences due to orientation were most evident on backgrounds where the moth had a high mean probability of being missed. Pietrewicz \& Kamil (1977) similarly showed that orientation had an effect on survivorship, but only when the moths were presented against those tree species where they were hard to detect. Here, I have shown that a similar phenomenon can arise within a single tree species that exhibits intraspecific variation in appearance. These results link well to the findings of Merilaita (2003) who argued that 'the difficulty of a detection task is related to the visual complexity of the habitat', so that prey might find it easier to evolve ways of reduced detection in more visually complex backgrounds. Animals other than moths may also enhance their background matching through appropriate choice of resting orientation. However, my experiments suggest that selection will only act on such behaviour when (i) the detection task is generally difficult and (ii) the background has some form of directional based pattern, generating an advantage from alignment.

One of the potential disadvantages of using a human predator system is that it ignores potential colour pattern information that is beyond human sensory perception, such as UV coloration (Cuthill et al., 2000). Although tree trunks generally have a low 
UV content,avian predators may still perceive the hue of these backgrounds differently compared with trichromatic humans (Hart and Hunt, 2007). While the use of human predator systems has been shown to produce results that roughly correspond to field survivorship measures (e.g. Fraser et al. 2007; Cuthill \& Szekely 2009), one should be careful not to over-extrapolate results from human predators to natural predators.

Two of the main principles of camouflage, disruptive coloration and background matching, operate synergistically; behaviourally mediated crypsis therefore has an influence on disruptively patterned species. Clearly, an improvement in background matching — derived from behavioural alignment—will make a disruptively coloured animal harder to detect. There are many types of proposed disruptive markings: edgeintersecting patches that disrupt shape, disruptive lines that bisect uniformity, disruptive eye stripes and coincidental disruption (Fig. 3.1). It is plausible that this effect could be employed by disruptively colored animals as an interesting behavior. It is likely that animals with disruptive lines are most sensitive to changes in their conspicuousness at different body orientations. And consequently they would benefit greatly from aligning their body patterns with the directionality of backgrounds. Further, when trying to measure the effect of disruptive coloration on camouflage (the objective of the remainder of this thesis), it will be important to control for potentially confounding influence of these lines, as they resemble background patterns and could enhance crypsis through matching pattern instead of preventing recognition through disruption.

Moth resting orientation on trees in the field varies, and can be non-random depending on moth species. Although orientation had been suggested to enhance moths' 
camouflage, I am not aware of any experiments that have tested precisely why moths experience the reduced detection rates from particular orientations. Here, I combined field data on moths' in situ orientation with an investigation of the benefits of orientation in two moth species, using a human predator system. Not only did the moths' orientation have an effect on survivorship, this effect could be linked directly to how the background tree image was presented (vertical or horizontally flipped) and how the moths themselves were patterned. Collectively, these data provide support for moths' natural orientation behaviour functions to enhance their background matching.

In subsequent data Chapters 4 and 5 a similar approach is taken, but instead of identifying background matching through a dependency on background properties (achieved here through rotating trees), I seek to identify disruptive coloration through a dependency on shape and boundary visibility. 


\section{Chapter 4: Disruptive coloration and the role of shape perception in}

\section{animal camouflage}

"There are certain inherent characteristics of the outline in many animals which greatly facilitate recognition by sight namely, regularity of form [aka shape]... It follows that any device which destroys or subdues these qualities will increase the difficulty of detection. Among many of the most perfectly camouflaged animals, this problem has been met by the modification of the contour itself. In fact, we see in this connexion two complementary methods of concealment in use among animals. In some cases... the outline is obscured by an interrupted marginal pattern: in others it is effaced by an irregular marginal form." (Cott, 1940)

\subsection{Abstract}

For over 100 years, it has been speculated that disruptively coloured edge markings on an animal's body mask its characteristic shape, thereby impairing its recognition. However, direct tests of the mechanism are rare. Here, I test whether disruptive coloration degrades shape information by presenting artificial moth-like targets to human subjects (as proxy visual predators) in a computer foraging experiment. The moth targets not only varied in colour pattern (presence/ absence of edge-intersecting patches) but also in shape outline (straight edge, high-frequency edge, low-frequency edge). I predicted that targets with straight edges would have more salient outlines, and therefore lower survival, than targets with ruffled edges (due to the visual mechanisms of edge detection and contour integration. The shape of moth-like targets affected their detectability as expected. I further predicted that edge coloration should decrease the likelihood of a target being detected, and that the magnitude of the concealing edge patches should diminish for shapes that are less detectable. Indeed, moth edge coloration provided concealment dependent on targets' shape, with straight-lined shapes benefiting more from edge markings than targets with low-frequency ruffled outlines. This shape * edge coloration interaction supports the hypothesis that edge-intersecting patches interfere with shape perception. 


\subsection{Introduction}

\subsubsection{Animal camouflage}

Camouflage reduces the likelihood of an object being detected and recognized. These complementary components of camouflage are manifested in two broad camouflage strategies: concealment by background matching and impairment of object recognition by countershading, masquerade or disruptive coloration. Concealment by background matching is achieved when animal's coloration resembles the colours and textures of its environment (Cott, 1940, Endler, 1984, Ruxton et al., 2004b). Whilst background matching is ubiquitous in nature, it has several disadvantages, such as being restricted to a relatively narrow range of backgrounds (Ruxton et al., 2004a, Merilaita et al., 1999). Even on backgrounds where high fidelity colour and texture matching is achieved, discontinuities between an animal's body outline and its background create conspicuous cues. The visual and cognitive systems of vertebrate predators are able to detect the boundaries (edges) of a prey's body. The more a prey's outline is revealed by edge detection, the more likely the predator(s) are to recognise the prey (Osorio and Srinivasan, 1991). To counter the shortcomings of background matching, an animal can hinder its recognisability in a number of ways: (i) masking the three dimensionality of its body via countershading (Stoner et al., 2003, Rowland et al., 2007, Kiltie, 1988, Edmunds and Dewhirst, 1994, Allen et al., 2012), (ii) masquerading as an unappealing or uninteresting object (Skelhorn et al., 2011, Skelhorn et al., 2010, Skelhorn and Ruxton, 2010) and (iii) employing disruptive coloration to mask its body outline by distorting its edges (Thayer, 1896, Cott, 1940, Cuthill et al., 2005). 
The potential contribution of disruptive coloration to camouflage went untested for nearly 100 years and its underlying mechanism(s) remain poorly understood. In Cuthill et al.'s (2005) pioneering study, artificial moth targets that overlaid mealworm baits were presented in the field and subject to attack by avian predators. Their results demonstrated a large survival benefit to edge-patterned targets. Subsequent studies have addressed the phenomenon of disruptive coloration by providing evidence for the effectiveness of edge disruption (Chiao et al., 2005, Cuthill et al., 2005, Cuthill et al., 2000, Endler, 2006, Fraser et al., 2007, Kelman et al., 2007, Mathger et al., 2007, Merilaita, 1998, Schaefer and Stobbe, 2006, Stevens and Cuthill, 2006, Stevens et al., 2006a, Stevens et al., 2006b, Stevens and Merilaita, 2009), coincidental disruption (Cuthill and Szekely, 2009) and disruption facilitating habitat generalism (Schaefer and Stobbe, 2006). Although these studies demonstrate that targets with edge markings have higher survivorship than those without, it is not entirely clear whether this result can be attributed to enhanced disruption, or to enhanced background matching. Therefore, to substantiate claims that edge coloration provides disruptive coloration, other forms of evidence for a disruptive function are required.

\subsubsection{Animal shape and concealment}

An animal's shape plays an important and somewhat overlooked role in predation, not only in escape (Lundvall et al., 1999, Van Buskirk and McCollum, 2000, Dayton et al., 2005, Langerhans, 2009) but also in detection. Thayer boldly stated that "Outlines of an animal's body tend to reveal it to the eyes of enemies" (Thayer, 1909). Since Thayer, a body of evidence has emerged suggesting that shape is of key importance to object 
recognition (Delvenne and Dent, 2008, Liebe et al., 2009, Soto and Wasserman, 2012) and particularly for animal recognition (Lloyd-Jones et al., 2010, Lloyd-Jones and Luckhurst, 2002, Elder and Velisavljevic, 2009). For example, novel shapes are known to be more difficult to detect and to require a learning period before being consistently recognized (Kourtzi et al., 2005, Dawkins, 1971). Further, recognition of shape can contribute to detectability independently of an object's colour (Ings et al., 2012, Brown et al., 1992, Carlile et al., 2006, Schluessel et al., 2012, Lehrer and Campan, 2004), with shape properties relied on for recognition when colour polymorphism occurs (Tanaka and Presnell, 1999). Therefore, an animal's shape influences its ability to conceal itself and may have evolved characteristics to minimise its detection (Nuernberg et al., 2012, Smith, 2003).

To determine what types of outline make a shape less detectable, it is important to examine the process of shape perception and the limits of biological vision. Shape perception is both computationally and attention-demanding (Elder et al., 2003) especially in cases of insufficient contrast between the animal's boundaries and its background (Marr and Hildreth, 1980, Stevens and Cuthill, 2006) and partial occlusion of the animal's shape by foreground objects (Tvardikova and Fuchs, 2010). To combat incomplete boundary information, shape perception has been shown to (i) detect edges, (ii) group detected edges into a boundary (or contour) and (iii) fill-in the missing pieces to estimate objects' shape. Aggregation of detected edges into a boundary is simple when there are a few, long, straight sides to the object's shape. However, as an object's shape becomes increasingly curvilinear, with many small sides that often change in their orientation, boundaries become less visually pronounced (Kennedy and Domander, 1985, 
Panis et al., 2008). Biological vision can process straight line boundaries, with coarse low spatial frequency visual clues, more rapidly than curvilinear edges (Panis and Wagemans, 2009). Once portions of boundaries are detected, visual systems rely on extrapolation of shape (Anderson et al., 2002, Kellman and Shipley, 1991). As an object's shape changes from straight to curvilinear, it also becomes increasingly difficult to reconstruct its outline from detected shape fragments (Singh and Fulvio, 2005, Singh, 2004). In short, increasing the number of sides and curvature of an object's boundaries hinders shape recognition.

Appreciating the mechanisms of shape perception leads us to make predictions about the detectability of animal shapes, which have long been postulated by naturalists (Field et al., 1993). Similarly, in military camouflage, the tassels on a ghillie suit serve to break up the silhouette of a person (Appendix Fig. D.1; (Behrens, 2009)), without reliance on disruptive markings to break up their outline. In the current study, I predict that moth-like triangles with ruffled sides should survive longer than those with straight sides. Indeed, Thayer noted "Lepidoptera's [shape] modifications ... of the outer edges of the wings... are cut-in, in gentle curves or sharp, angular notches, ... altered much and variously from the simple, average butterfly-shape." (Thayer (1909); Fig. 4.1). Whilst the shape of moth wings affects their aerodynamics (Betts and Wootton, 1988, Vogel, 1994), it is possible that shape might also increase concealment. One might speculate that such edge ruffles in many taxa might have evolved ghillie suit-like ruffled edges for the purpose of concealment. Here, I test if such ruffled edge boundaries enhance the survivorship of moth targets in a visual search task using human subjects as visual 'predators'. 

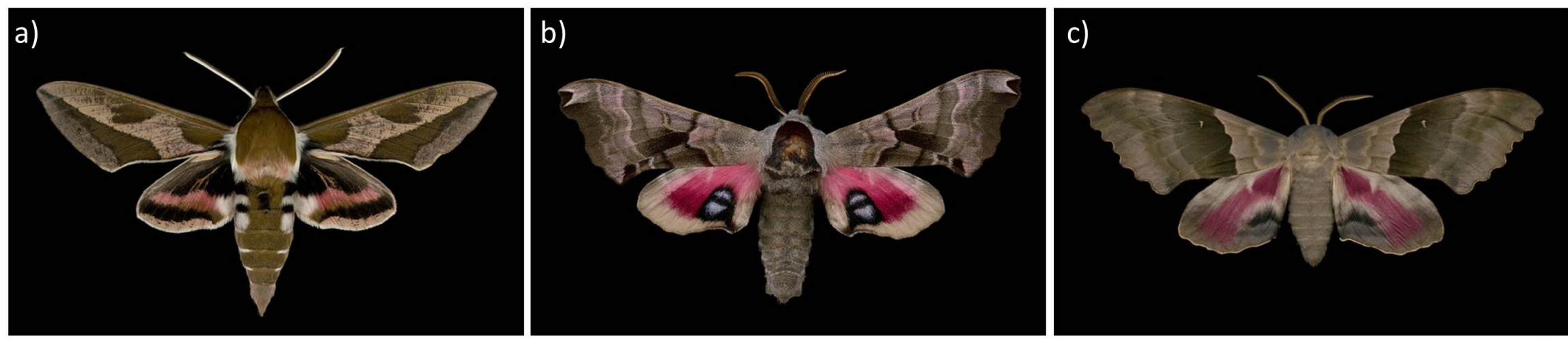

Figure 4.1: Three sphinx moths that show a diverse range in wing shape. Forewings trialing margin can be either $(a)$ straight like the Spurge Sphinx Moth Hyles euphorbiae or $(b)$ ruffled like the Twin-spotted Sphinx Moth Smerinthus jamaicensis and (c) the Big Poplar Sphinx Moth Pachysphinx modesta. Photographs by Jim des Rivières (moths.ca). 


\subsubsection{Shape and edge coloration interaction}

Here, I ask if the value of disruption is in any way dependent on the bearer's shape. It has long been speculated that disruptive coloration acts to distort shape perception (Wilson and Keil, 2001) by obscuring an animal's characteristic outline (Thayer, 1909, Cott, 1940, Ruxton et al., 2004a). If the survival value of edge markings covaries with shape, then this would suggest that edge markings provide camouflage through a visual mechanism related to the properties of the object's shape. Such a result could not be an artefact of background matching, but rather due to a distinct disruptive function of edge markings. Evidence of animal camouflage dependency on shape would provide a mode of action for disruption and validate claims that edge markings indeed function as disruptive coloration.

To test if disruptive coloration affects shape perception, I compared the survival benefit of edge coloration on moth-like targets of varying shapes. I predicted that where outlines are easy to detect they should have a correspondingly large benefit from disruptive coloration; whereas outlines that are already difficult to detect will gain a smaller benefit from disruptive coloration. Thus, an interaction between shape and edge coloration would provide support for disruptive coloration affecting shape perception (Fig. 4.2). Where shape is a highly distinguishing feature of an object, the potential for disruptive coloration to reduce the visibility of the object's shape may be considerable. Alternatively, where shape is a less prominent feature (for instance, due to a ruffled outline), then disruptive coloration may still reduce overall visibility, but its net benefit would be expected to be lessened. Conversely, if disruptive markings enhance 
camouflage by means other than shape distortion, then the benefit of disruptive markings would be unaffected by variation in shape.

I undertook a human-computer foraging experiment to test if the benefit of disruptive markings varies according to shape's detectability. A 2 x 3 factorial design was used to manipulate moth target coloration (inside pattern, edge-disruptive pattern) and shape (straight edged, high-frequency edged, low-frequency edged). Of these shape treatments, I predicted that straight-edged moth targets would have lower survival than the 'ruffled' curvilinear high-frequency edged and low-frequency edged moth targets. I then investigated how the advantage of disruption changes with shapes of low and high complexity. I predicted an interaction between shape and edge coloration, anticipating that disruption would provide a larger survival advantage for shapes with straight edges compared to shapes with ruffled edges. 

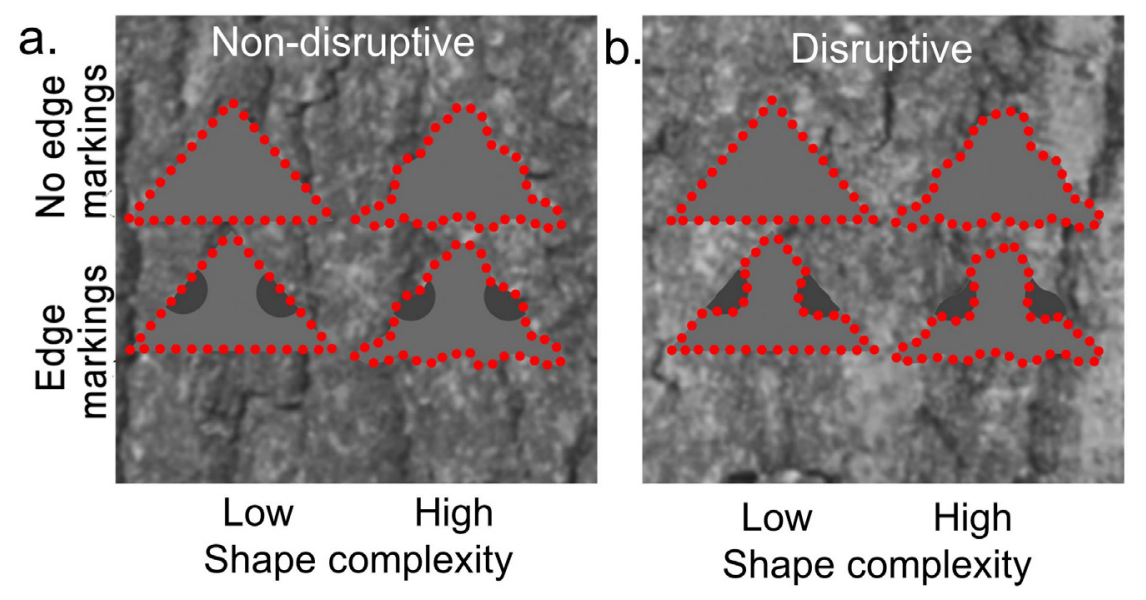

C.

d.

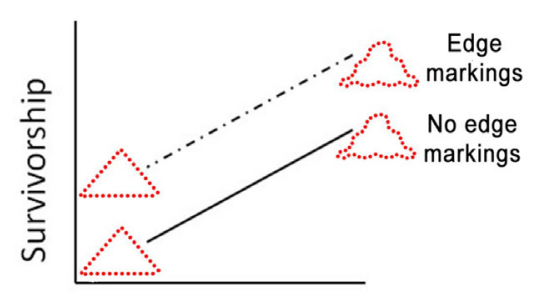

Shape complexity

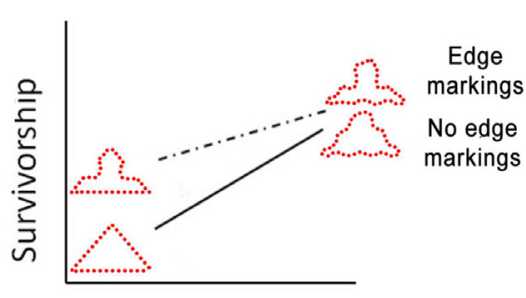

Shape complexity

Figure 4.2: Edge markings could either function by background matching or disruption (whereby shape perception is altered). If edge markings were to function through $(a)$ background matching, perceived shape (represented by the red dashed lines) does not change with the addition of edge markings. In contrast, if edge markings were to function through $(b)$ disruption, perceived shape is predicted to change (NB. $a \& b$ are idealized / simplified targets as they lacking non-edge pattern; they were not used in this experiment). Next, I provide graphical illustrations of how edge markings and targets' shape covary to effect survivorship, according to their above models of edge markings function. (c) Background matching edge markings would show an additive affect, independent of shape distortion, while edge markings with a $(d)$ disruptive function would be predicted to interact with the physical properties of shape that influence detectability. 


\subsection{Methods}

\subsubsection{Experimental protocol}

A Microsoft Visual Basic Express ${ }^{\circledR} 2008$ program presented cryptic moth targets super-imposed on photographs of trees to volunteer human subjects (similar to Webster et al. 2009). All participants were visitors to Carleton University’s Maxwell MacOdrum Library, where the testing took place. Computer monitors were rotated to a portrait orientation to show high-resolution gray scaled ${ }^{7}$ tree images $(1600 \times 800$ pixels $)$ with or without triangular moth targets (60 high x 100 wide pixels) on them. Monitors were positioned $3 \mathrm{~m}$ away from subjects, rendering the visual angle of the target $0.35^{\circ}$ high by $0.73^{\circ}$ wide. Several training screens were presented to individual subjects, pre-exposing them to each treatment. I then orally explained to each subject, using a consistent script, that he/she was required to click on the moth targets as fast as possible and that, once a target had been successfully clicked on (or when the subject was satisfied that no target was present), the subject could proceed to the next screen by pressing a button in the top left-hand corner. This allowed for a self-determined break period before continuing with their visual search. Either one moth or no moth was presented per tree background during the search task. I operationalize detectability using two metrics; survivorship is defined as the proportion of moths that went undiscovered per treatment, and search time is defined as of those targets detected, the time (in seconds) to locate and click upon them.

\footnotetext{
${ }^{7}$ By presenting targets on gray scaled tree photographs a perceptual dimension of the background is removed. These camouflaged targets become more difficult to discover on the grayscale background, subsequently making the task more ecologically valid.
} 
Digital photographs of sugar maple (Acer saccharum) trunks were thresholded at their median grayscale value and used to derive the targets. Thresholded areas were then coloured dark and light gray based on the original tree image's 25th and 75th grayscale percentiles using Adobe Photoshop ${ }^{\circledR}$ (Abode Systems Inc., San Jose, CA, USA). Triangular areas from these bi-coloured tree images were selected. The triangles left half were mirrored about the vertical axis of symmetry. These bi-coloured triangles with vertical symmetry were designed to simulate moths on a tree trunk.

\subsubsection{Target treatments}

The experiment consisted of six target treatments in a $2 \times 3$ factorial design with two main factors: coloration (inside pattern, edge-interceptive pattern) and shape (straight edged, high frequency edged, low frequency edged; see Fig 4.3). Targets with edgeintersecting patches were considered as putative disruptively colored (Cuthill et al., 2005). Presence of edge-intersecting patches was defined as a target with five or more continuous dark pixels that touch the target's outline and absence of edge intersecting patches had no dark pixels touching its outline. For both these edge coloration treatments, 10 unique base patterns were used. This within-treatment replication ensured that results would be generalizable about the presence/absence of edge patches. The constraint of 10 replicates per coloration treatment ensured that the experiment did not exceed 15 minutes per subject (to maintain subject motivation during the computer foraging experiment).

To my knowledge, shape properties have not previously been studied in relation to an animal's detectability. My artificial moth targets had ruffled edges on all three sides 
of their triangular base shape. In moths, edge modifications are normally restricted to the outer margin; here, I have chosen to manipulate all sides (Fig. 4.3) in an effort to magnify any effect of edge composition. As I was unsure what degree of edge boundary manipulation might affect perceived outline break up, I explored one straight edge shape and two ruffled edged shapes inspired from Ottawa Valley (Ontario, Canada) moths: (1) low-frequency edged, similar to Smerinthus jamaicensis, Plagodis alcoolaria, Drepana bilineata and (2) high-frequency edged, similar to Pachysphinx modesta, Zale lunata, Catocala unijuga, Lytrosis unitaria (Fig. 4.1). While expecting a detection difference between straight and ruffled shapes, I had no expectation as to detection differences between high- and low-ruffled edged shapes. 


\section{Shape}

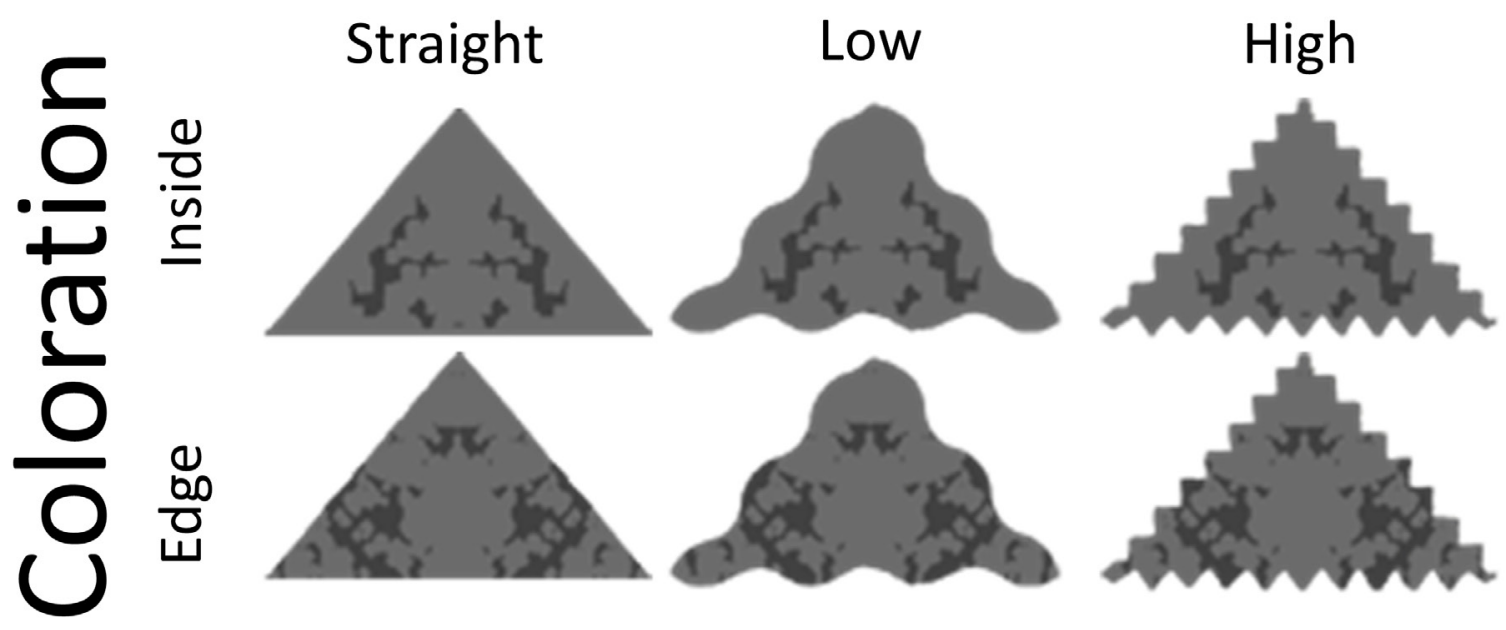

Figure 4.3: Factorial design of artificial moth target treatments, varying shape (straight, low-frequency and high-frequency edged) and coloration (edge-intersecting patches and inside patches). 


\subsubsection{Statistical analysis}

Statistical analyses were performed in SPSS version 17 (SPSS Inc., Chicago). General linear mixed models (GLMM) were fitted to analyse the data for search time, with subjectID, tree background and targetID being treated as random factors; shape and edge coloration were treated as fixed factors and $x-y$ screen coordinates as covariates. To test my a priori prediction, I included a shape*edge coloration interaction term in the fitted model. Search time had a normal distribution once double log (log-log) transformed, whilst survivorship had a binomial distribution and so a Generalized Linear Mixed Model (GzLMM) logistic regression was appropriate.

\subsection{Results}

\subsubsection{Animal coloration}

There was a main effect of edge coloration treatment (disruption / no disruption) on moth target survivorship $\left(\mathrm{W}_{1,4018}=62.81, p \leq 0.001\right.$; Table 4.1$)$ and subject search time $\left(\mathrm{F}_{1,2985}=32.17, p \leq 0.001\right.$; Table 4.2). The presence of edge-intersecting patches is associated with heightened survivorship and, of those targets discovered, a longer search time.

\subsubsection{Animal shape and concealment}

There was a significant treatment effect of shape on survivorship $\left(\mathrm{W}_{1,4017}=26.95\right.$, $p<0.001$; Table 4.1) and search time $\left(\mathrm{F}_{1,2984}=8.32, p<0.001\right.$; Table 4.2). A post hoc Tukey test was performed to determine the difference in subject search time between my three shape treatments (straight, low-frequency and high-frequency). Of those targets that 
were found, straight shaped targets were found more rapidly than low-frequency shapes $(\mathrm{p}<0.001)$; straight and high-frequency shape showed a non-significant borderline difference $(p=0.081)$; and there was no significant $(p=0.172)$ difference in the time to detection of low- and high-frequency targets (both ruffled boundary treatments).

\subsubsection{Shape * edge coloration interaction}

From visually inspecting Fig. 4.4, it seems that the effect of edge coloration on the detectability of moth targets is dependent on target shape and vice versa. This edge coloration* shape interaction had a non-significant borderline effect for both survivorship $\left(\mathrm{W}_{1,4017}=5.56, p<0.062\right.$; Table $4.1 \&$ Fig. $\left.4.4 \mathrm{a}\right)$ and search time $\left(\mathrm{F}_{1,2984}=2.56, p<\right.$ 0.077; Table 4.1 \& Fig. 4.4b). Because I was only interested in this interaction term between shapes of varying detectability, the data were split into two subsets, allowing the comparison of the straight shape treatment separately with each of my ruffled shape treatments (straight vs low-frequency and straight vs high-frequency). This approach was informed by my post hoc Tukey test of shape (shown above), which found no difference in detectability between ruffled shaped targets, such that no interaction would be expected for this pair. Between straight and low-frequency shape there was a significant interaction with the response variable of survivorship $\left(\mathrm{W}_{1,2599}=5.27, p<0.022\right)$ and search time $\left(\mathrm{F}_{1,2016}=5.23, p<0.022\right)$. In contrast, the interaction between straight and high-frequency shape shows a non-significant (although borderline) interaction for survivorship $\left(\mathrm{W}_{1,2599}=5.26, p<0.074\right)$ and search time $\left(\mathrm{F}_{1,2017}=2.21, p<0.137\right)$. 

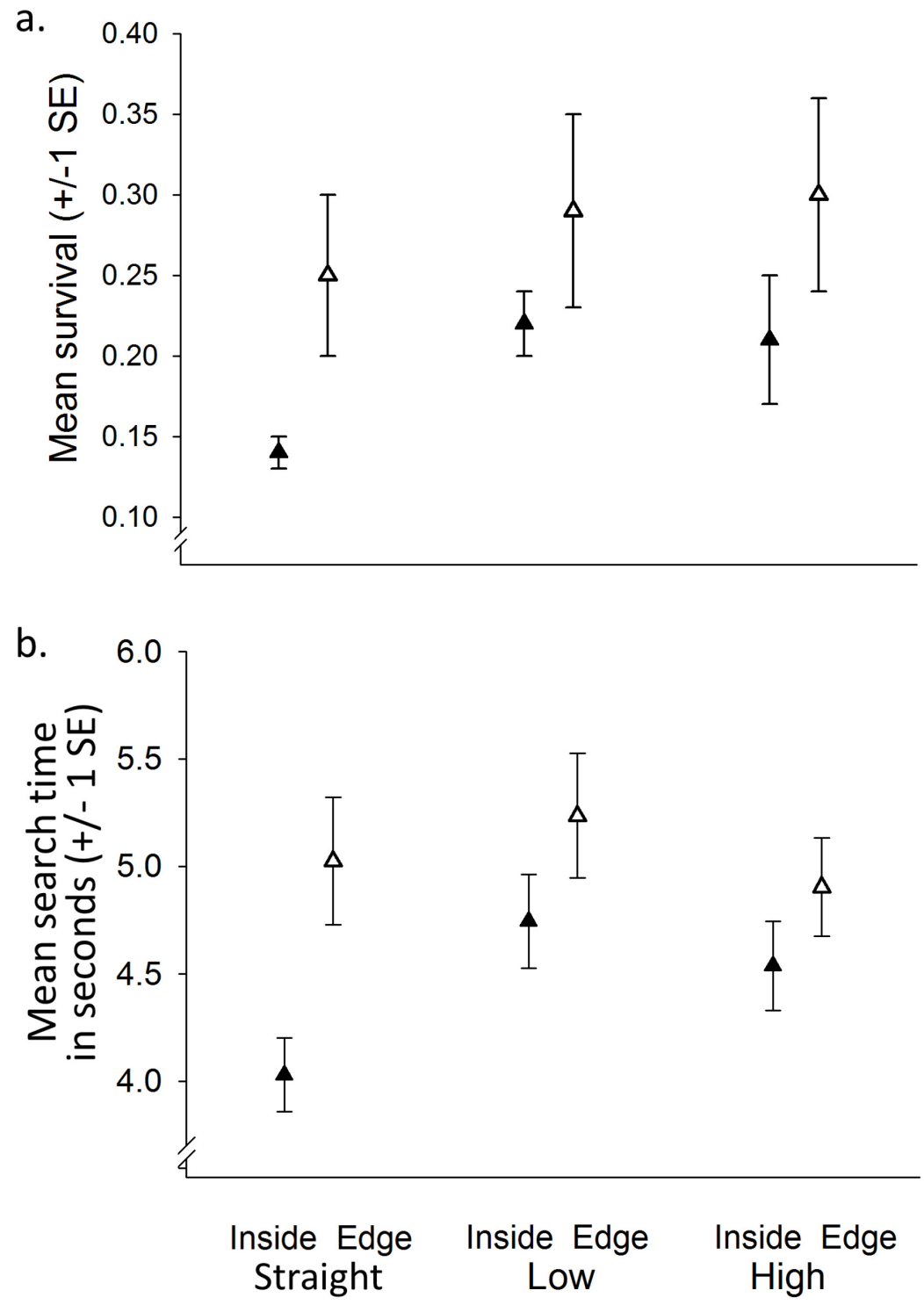

Figure 4.4: Detectability of moth targets, which differ in shape and disruptive patterning. Edge intersecting patches increase (a) survivorship and $(b)$ search time for straight shape, but its benefits are diminished for ruffled shaped (Low-and High-frequency edged). Closed triangle symbols denote inside patterned targets (which lack edge markings), whereas open triangles denote targets with edge-intersecting patches. 
Tables 4.1: Results of the logistic regression model testing for the effects of factors on the survivorship of moth targets under 'predation' by human subjects. The results of this logistic regression are presented. The random factors of SubjectID, Tree background and TargetID were controlled for in the model. Cells reporting results are formatted as follows: Wald Chi-squared test statistic, degrees of freedom, and p-value. The column Full represents analysis of the full dataset, and columns Straight vs. Low and Straight vs. High represent subsets of the data to consider the straight shape pair against each of the other ruffled shapes. The sample size for each analysis were: Full $(n=4019)$, Straight vs. Low $(\mathrm{n}=2600)$ and Straight vs. High $(\mathrm{n}=2600)$.

\begin{tabular}{|c|c|c|c|}
\hline \multirow[t]{2}{*}{ Predictors } & \multicolumn{3}{|c|}{ Dependent: Survivorship } \\
\hline & Full & Straight vs. Low & Straight vs. High \\
\hline Edge coloration & $62.81, \quad 1, \quad p \leq 0.001$ & $47.71, \quad 1, \quad p \leq 0.001$ & $52.57, \quad 1, \quad p \leq 0.001$ \\
\hline Shape & $26.95, \quad 2, \quad p \leq 0.001$ & $20.85, \quad 1, \quad p \leq 0.001$ & $21.94, \quad 1, \quad p \leq 0.001$ \\
\hline SubjectID & $346.88,63, p \leq 0.001$ & $250.54,63, p \leq 0.001$ & $232.16,63, p \leq 0.001$ \\
\hline Tree & $245.93,9, \quad p \leq 0.001$ & $196.44,9, \quad p \leq 0.001$ & $149.98,9, \quad p \leq 0.001$ \\
\hline TargetID & $239.40,19, p \leq 0.001$ & $153.60,19, p \leq 0.001$ & $157.62,19, p \leq 0.001$ \\
\hline Y-coordinate & $1.73, \quad 1, \quad p=0.189$ & $0.01, \quad 1, \quad p=0.904$ & $2.58, \quad 1, \quad p=0.109$ \\
\hline X-coordinate & $0.01, \quad 1, \quad p=0.189$ & $0.08, \quad 1, \quad p=0.928$ & $0.53, \quad 1, \quad p=0.465$ \\
\hline $\begin{array}{l}\text { Edge colour * } \\
\text { shape }\end{array}$ & $5.56, \quad 2, \quad p \leq 0.062$ & $5.27, \quad 1, \quad p \leq 0.022$ & $3.18, \quad 1, \quad p \leq 0.074$ \\
\hline
\end{tabular}


Tables 4.2: Results of the general linear mixed model testing for the effects of factors on search time of human subjects 'foraging' for moth targets. The random factors of SubjectID, Tree background and TargetID are controlled for in the model. Cells reporting result are formatteds follows: F test statistic, degrees of freedom and p-value. The column Full represents analysis of the full dataset, and columns Straight vs. Low and Straight vs. High represent subset of the the data to consider the straight shape pair against each of the ruffled shapes. The sample size for each analysis were: Full $(n=2987)$, Straight vs. Low $(n=2017)$ and Straight vs. High $(n=2018)$.

\begin{tabular}{|c|c|c|c|}
\hline \multirow[t]{2}{*}{ Predictors } & \multicolumn{3}{|c|}{ Dependent: Search time Log Log transformed } \\
\hline & Full & Straight vs. Low & Straight vs. High \\
\hline Edge coloration & $32.17,1, p \leq 0.001$ & $23.99,1, p \leq 0.001$ & $29.18,1, \quad p \leq 0.001$ \\
\hline Shape & $8.32, \quad 2, \quad p \leq 0.001$ & $16.94,1, p \leq 0.001$ & $5.83,1, \quad p=0.016$ \\
\hline SubjectID & $7.96, \quad 63, p \leq 0.001$ & $5.64, \quad 63, p \leq 0.001$ & $5.57, \quad 63, p \leq 0.001$ \\
\hline Tree & $12.54,9, \quad p \leq 0.001$ & $7.99, \quad 9, \quad p \leq 0.001$ & $8.95, \quad 9, \quad p \leq 0.001$ \\
\hline TargetID & $11.81,19, p \leq 0.001$ & $7.97, \quad 19, p \leq 0.001$ & $7.87, \quad 19, p \leq 0.001$ \\
\hline Y-coordinate & $51.12,1, p \leq 0.001$ & $33.05,1, p \leq 0.001$ & $39.61,1, \quad p \leq 0.001$ \\
\hline X-coordinate & $0.29, \quad 1, p=0.588$ & $0.86, \quad 1, p=0.353$ & $0.28, \quad 1, \quad p=0.597$ \\
\hline $\begin{array}{l}\text { Edge colour * } \\
\text { shape }\end{array}$ & $2.56, \quad 2, \quad p \leq 0.077$ & $5.23, \quad 1, p \leq 0.022$ & $2.21, \quad 1, \quad p \leq 0.137$ \\
\hline
\end{tabular}




\subsection{Discussion}

\subsubsection{Animal coloration}

Although many recent studies have shown that edge-intersecting colour patches are associated with heightened survivorship in animals (Cuthill et al., 2005, Cuthill et al., 2000, Schaefer and Stobbe, 2006), these findings provide just one source of evidence for a function of disruptive coloration. Here, I tested another prediction related to disruptive coloration, namely that it should be more effective in reducing the detection rates of targets with simple shapes compared to targets with complex shapes.

\subsubsection{Animal shape and concealment}

As expected, targets with ruffled edges were more difficult to detect than straightedge targets (Table 4.1). Biological vision performs more effectively when detecting straight edges compared to curvilinear edges (Bell et al., 2010); therefore, shapes that are made up of straight edges have more noticeable outlines. I had no a priori predictions regarding the detectability of the two non-straight target shapes used in the current study. High- and low-frequency edged targets had similar survivorships; however, for those targets that were detected, low-frequency edge targets required a longer search time. Future work should investigate which boundary properties explain the detectability of shapes.

My findings show that shape plays an important role in concealment and has implications for the evolution of anti-predator defenses. Shape provides additional concealment by rendering outlines more difficult to detect. When camouflage is less 
effective (e.g. in visual environments with low complexity (Merilaita, 2003)), shape might provide additional concealment. There is some limited evidence that shape has evolved as an adaptation for concealment (Nuernberg et al., 2012, Smith, 2003).

Obviously, for shape to evolve a concealment function, evolutionary trade-offs have to be made with factors like aerodynamics and structural integrity. The cost of such constraints could differ depending on the organism's life history. For example, in the context of shape concealment, an ambush predator would have fewer aerodynamic constraints owing to its hunting style than a hunt-on-the-wing predator. So, comparatively, the ambush predator might be more likely to evolve shape concealment. Alternatively, because the shape of moth wings affects flight behaviour (Betts and Wootton, 1988), it is possible that this feature has co-evolved to improve both aerodynamics and concealment. As aerodynamic performance has co-evolved with anti-predator defense in insects (Srygley, 2007), ruffled edges on wings might have similarly evolved to conceal body shape.

\subsubsection{Shape * edge coloration interaction}

In the current study, I provide the first empirical test of an interaction between the value of disruptive coloration with body shape. My a priori prediction was that edgeintersecting patches would have a large effect on reducing detectability of shapes that are most easily detected, and that this disruptive advantage diminishes as the target's shape becomes less detectable (where here, the addition of ruffles targets boundaries makes shapes less detectable). Indeed, when comparing straight and low-frequency edged shapes, I observed a large survivorship benefit of edge-intersecting markings on shapes 
with straight boundaries and a smaller benefit for shapes with low-frequency boundaries. An artefact of this shape dependency of disruptive coloration is that if the shape is already difficult to detect, then disruptive coloration has less to offer. This shape * edge coloration interaction is absent when comparing straight vs. high-frequency edge shapes. These results suggest that shape boundaries affect detectability, but only specific curvilinear shape boundaries reduce detection in a process similar to edge coloration. Therefore, the current study indicates that edge coloration is capable of affecting shape perception, as the benefit of having edge-intersecting patches is diminished on lowfrequency shapes compared to straight shapes. This provides a proof of the assertion that disruptive coloration plays a role in distorting shape perception.

Insight into the mechanism by which disruptive coloration distorts shape perception might be provided by differences between my ruffled shape treatments. The inconsistency with a shape * edge coloration interaction suggests that the low- and highruffled treatments in the current study might convey different shape information. These shapes differ in many properties, such as convexity (Rubin, 1915, Kanizsa and Gerbino, 1976, Bertamini and Lawson, 2008), compactness, curvature and spatial frequency. My study was not designed to disentangle these properties of shape (rather I asked if disruptive coloration is dependent on shape) and, as such, I can only speculate as to which shape properties underlie the efficacy of disruptive colorations. Disruptive coloration is seemingly ubiquitous in nature and has a substantial effect on detection (Cuthill et al., 2005): as such, disruptive coloration should distort shape information used by a diverse range of taxa and this shape information should be highly influential in rapid 
recognition. These requirements are satisfied by low spatial frequency visual cues, which facilitate the rapid identification of animal body outlines (Elder and Velisavljevic, 2009, Marotti et al., 2012). Perhaps the difference in low spatial frequencies shape information explains why only the low-ruffled boundary shape elicited a shape * edge coloration interaction, but high-ruffled boundary shape compared to straight shape does not (Appendix Fig. D.2). It is possible that disruptive coloration relies on distorting the shape of animals with low spatial frequency outlines, but further work is needed to test which global shape properties are exploited by disruptive coloration.

Acknowledging the role of shape perception in disruption allows us to extend the range of predictions regarding the functions of disruptive coloration. Species with simple shapes might be expected to have more disruptive colorations than species which mask shape detectability through their ruffled/curvilinear boundaries. To date, such betweenspecies phylogenetic tests for disruption have only included appearance characters (Allen et al., 2011, Hart and Hunt, 2007, Caro, 2011, Caro, 2005, Caro and Stankowich, 2010, Caro et al., 2012) and have provided limited insight into disruptive coloration due to the lack of functional predictions (Caro, 2009). It seems that there is stronger selection for disruption on shapes that have straight (or maybe simple low spatial frequency) edges and are easily recognised. Whilst this suggests that animals with easy-to-recognise shapes might have more disruptive coloration, other factors also affect this outcome (e.g. the selective advantage of avoiding detection and recognition, when accounting for other anti-predator strategies). Comparative studies of shape and disruptive coloration open 
new avenues of research into how disruptive coloration functions and the co-evolution of shape and camouflage colorations.

To generalise these results from humans to other animals, it is important to note that available evidence suggests that human and non-human visual and cognitive systems process shape in similar ways. There has been much debate about the exclusivity of humans to hold representations of objects. Whilst humans do indeed have high capacity for abstract representation, human and non-human shape perception in real-world visual tasks shows much consistency for behavioural outcomes and their mechanisms. A common challenge in shape perception is recognising incomplete shapes, such as objects that are occluded. Such objects can still be accurately recognised based on incomplete shape information in mammals (Barbet and Fagot, 2011, Parron and Fagot, 2007, Deruelle et al., 2000, Sato et al., 1997, Kurylo, 2008, Fujita, 2001, Kanizsa et al., 1993), birds (Nakamura et al., 2010, Nagasaka et al., 2005, Cavoto and Cook, 2006, Regolin and Vallortigara, 1995) and fishes (Truppa et al., 2010, Sovrano and Bisazza, 2008) (but see (Burke et al., 2001)). These results are particularly strong in ecologically meaningful experiments; for instance, blue tits (Parus caeruleus) can recognise and elicit antipredator behaviour towards occluded models of their predators (Tvardikova and Fuchs, 2010). Critical to shape perception is the mechanism of perceptual grouping (the grouping of detected edges into boundaries), which is well documented in humans (Wertheimer, 1923, Wagemans et al., 2012a, Wagemans et al., 2012b, Helson, 1933). The influential contribution of perceptual grouping to vision is evident in many visual illusions (Halko et al., 2008). Similarly, animals from diverse taxa also respond to visual illusions involving perceptual grouping (mammals (Spinozzi et al., 2009, Vonderheydt et al., 1984, Fagot and Tomonaga, 2001), birds (Nieder and Wagner, 1999, Zanforlin, 
1981), fishes (Wyzisk and Neumeyer, 2007, Sovrano and Bisazza, 2008) and insects (Horridge et al., 1992)). Within the context of the current study, a non-vertebrate animal, the cuttlefish (Sepia officinalis), provides a compelling example of the use of perceptual grouping to facilitate camouflage decisions (Zylinski et al., 2012). It is not just the behavioural outcomes that suggest similar shape processing between humans and nonhumans, but also neural mechanisms. When encountering an unknown or known object, both humans and non-humans utilize perceptual grouping to activate distinct neural architecture. Changing visual processing from bottom-up for unknown objects (located at the visual cortex V1) to top-down for known objects (Volberg et al., 2013) facilitates faster behavioural decisions. I therefore postulate that these results collectively might be generalizable from humans to vertebrate predators.

Disruptive coloration has many functional predictions that can be used to distinguish it from background matching (Stevens, 2007). Disruptive targets (sensu stricto) should take longer to be recognised, due to shape recognition being impaired. Surprisingly, many studies claim that a coloration is disruptive, whilst failing to test essential functional predictions (but see Stevens \& Cuthill 2006, Webster et al. 2013). My novel method of varying both shape and edge markings infers a functional difference, circumventing the methodological difficulties of measuring object recognition. Finally, shapes containing straight-lined boundaries will have highly noticeable edges, and stand to gain a lot from disruptive coloration, whereas certain ruffled edge boundary shapes that have less diagnosable edges and should benefit much less from disruption. My study provides the first empirical test of Thayer's century old question, that disruptive coloration provides camouflage through distorting shape perception. 


\section{Chapter 5: Disruptive coloration and boundary visibility: Testing functional predictions of disruptive coloration}

"Every solid object has a boundary... and while this boundary occupies no room itself, it has a recognizable form, and it is, indeed, one of the most important factors enabling one to recognize any familiar body. It therefore follows that camouflage of the contour ... will demand special attention in any satisfactory scheme of camouflage." (Cott, 1940)

\subsection{Abstract}

Disruptive coloration is a camouflage strategy that is proposed to function by breaking up an animal's outline, thereby impairing its recognition. The emerging literature on disruptive coloration has consistently shown an association between putative 'disruptive' edge coloration and heightened survivorship. These results raise two related questions. First, how does edge coloration enhance survival and, second, is this heightened camouflage attributable to a disruptive function? If edge coloration elevates survivorship through outline disruption, then this camouflage strategy should depend on the visibility of boundaries. Therefore, disruptive function can be ascribed if the benefits of edge coloration are conditional upon boundary visibility. To test if concealment due to edge coloration is dependent on edge visibility, I used a $2 \times 2$ factorial design to investigate the influence of coloration (inside pattern, edge-intersecting pattern) and boundary visibility (control, translucent boundary) on artificial moth survivorship in a human computer foraging task. Translucent boundaries are predicted to increase survivorship of targets, because they are less visible. However, if edge markings are disruptive, then it is also predicted that boundary treatment will interact with edge coloration. Conversely, if edge 
markings enhance survivorship by facilitating background matching (and not disruptive coloration), then it is predicted that no interaction will be observed. As expected, I

observed the former: boundary visibility and edge coloration interacted and affected both survivorship and search time. Indeed, these interactions were synergistic, with translucent boundaries and edge coloration combining to further conceal moth targets. These results suggest that edge-intersecting patches act to lower detection rates through a disruptive function.

\subsection{Introduction}

\subsubsection{Background matching and disruptive coloration}

Camouflage coloration serves to conceal organisms. Two broad strategies that contribute to an organism's camouflage are: (1) the capacity to blend in to an environment by background matching, and (2) the ability to impair recognition by predators by countershading, masquerading, and disruptive coloration through masking three-dimensionality, resembling inedible objects, and breaking up distinctive outlines, respectively. While background matching is well studied (Endler, 1984, Ahnesjo and Forsman, 2006, Chiao et al., 2009, Dimitrova and Merilaita, 2012, Caro, 2005, Caro et al., 2012), the extent to which an animal's recognisability influences the effectiveness of its camouflage is still poorly understood, particularly for disruptive coloration (Stevens and Merilaita, 2009, Stevens and Cuthill, 2006).

Thayer (Thayer, 1909) and Cott (Cott, 1940) proposed that disruptive markings could contribute to camouflage by breaking up salient features (such as limbs, eyes, or an 
animal's entire outline) that are necessary for the identification of an organism. The visual perception of the shape of prey by predators could be distorted by: (1) edge markings that fragment the prey's outline, or (2) bisecting stripes that create false boundaries across the preys' surface (thereby creating an outline that is uncharacteristic or misleading). Disruptive coloration also affects features that are not part of the prey's whole outline (Fig. 2.1), but are nevertheless important for its identification. These include markings that obscure facial features (e.g. eye stripes) or mask appendages (e.g. coincidental disruption (Cuthill and Szekely, 2009)). Of these possible disruptive markings, edge coloration has received the most attention; maybe this is because boundaries properties are known to provide important visual cues for object recognition (Troscianko et al., 2009, Elder and Velisavljevic, 2009, Soto and Wasserman, 2012)'(Jones et al., 1997). Since highly visible boundaries are an important contribution to object recognition, targets with low boundary visibility will have less to gain from edge disruption than targets with high boundary visibility. This dependency of edge markings performance on boundary visibility is an artefact of disruptive coloration's function and can be exploited to identify whether or not edge markings are disruptive. Here, I seek to expand upon previous work by testing if the effect of edge coloration on rates of detection (Cuthill et al., 2005, Fraser et al., 2007, Merilaita and Lind, 2005, Stevens et al., 2006b, Schaefer and Stobbe, 2006, Cuthill et al., 2000, Cuthill and Szekely, 2009, Stevens et al., 2009, Chapter 4) is dependent on the visibility of an animal's boundary. Evidence of such an effect is necessary to support the hypothesis that edge patches are a form of disruptive coloration that function by distorting characteristic outlines. 
There is growing evidence that the presence of edge coloration augments survivorship, and this has been interpreted as evidence for their disruptive function (Cuthill et al., 2005, Cuthill et al., 2006b, Cuthill and Szekely, 2009, Stevens et al., 2006b, Fraser et al., 2007, Stevens et al., 2009, Schaefer and Stobbe, 2006). However, this is indirect evidence of function because it does not directly address the mechanism(s) involved. Surprisingly, there are few empirical studies that verify how disruptive coloration functions (Stevens \& Cuthill 2006, Chapter 4). The first study to address functional questions about disruption was Stevens \& Cuthill (2006) (Stevens and Cuthill, 2006). They showed that edge-intersecting patches impede detection of edges. Whilst this is an important verification, it only considers low level visual processing (see Chapter 2.2.1a). However, while a change in edge detection might influence an outcome of object recognition (and detection rates), it also depends on other conditions affecting subsequent ‘downstream’ processes, such as contour integration, contour completion and identification (Fig. 2.3).

If edge coloration acts to disrupt object recognition, then camouflage due to edge coloration will be dependent on the visibility of the object's boundaries. Alternatively, if edge coloration achieves concealment as an artefact of background matching (and not through disruptive coloration), then a change in edge coloration should not interact with the boundary's visibility. Therefore, it is possible to test predictions of how disruptive coloration functions by observing differences in rates of detection between objects that have high and low boundary visibility with or without edge coloration. 


\subsubsection{Boundary visibility is operationalized through transparency}

Animal transparency is another camouflage strategy (Ruxton et al. 2004).

Background matching can be achieved by allowing light to pass through an organism, thereby minimizing its reflection. The more light that an organism reflects and scatters, the less translucent it is, which impedes concealment. Transparent objects automatically match their backgrounds, and their transparency makes their global shape difficult to recognise (Mcfall-Ngai, 1990, Johnsen, 2001) because of lower contrast between the background and the animal's boundaries, which renders edge detection less effective (Singh and Anderson, 2002b, Singh and Anderson, 2002a). These advantageous properties of transparency have likely contributed to its evolution in both pelagic and terrestrial environments; however, it has associated costs that might explain why other protective coloration strategies, like background matching and disruptive coloration, are more common. First, the effectiveness of concealment by transparent tissues diminishes with increasing diameter of the animal's body. When an animal is small, the degree of light scattering by the transparent tissue is low; however, when the same tissue is larger it scatters much more light, which makes it more visible. Consequently, transparency is less common in larger organisms, even when controlling for phylogeny (Johnsen, 2001).

Second, specific light conditions and background reflectance are needed to ensure that the reflectance of transparent animals is similar to their surroundings (Johnsen, 2001). Pelagic predators hunting for transparent prey exploit their preys' fixed refractive index by using bioluminscesent 'searchlights' (Kubodera et al., 2007, Widder, 2010) that glint when they intersect their prey against a dark background. Lastly, concealment of some transparent organisms is compromised when they consume non-transparent prey items 
(Giguère and Northcote, 1987). Transparent or semi-transparent Lepidoptera that might experience some of these costs include the ithomiid butterflies and sphingid moths (Yoshida et al., 1997, Binetti et al., 2009, Meyer-Rochow, 1974).

In the current study, I test the hypothesis that disruptive coloration is dependent on the visibility of other features (boundaries) used by predators for their identification of prey. 'Disruptive' edge patches are assumed to break-up a prey's boundary and thereby reduce its recognisability. I then operationalize boundary visibility as control (with 'high' visibility for a 'normal' boundary) and translucent (with 'low' boundary visibility). If edge coloration and boundary visibility interact, I predict that edge coloration increases survivorship (and its corollary, search time), but will also be dependent on the visibility of the target's boundary. To investigate the interplay between disruptive coloration and translucent boundaries, I conducted a human computer foraging experiment in which human subjects searched on computer screens for artificial moth-like targets.

\subsection{Methods}

A Microsoft Visual Basic Express ${ }^{\circledR} 2008$ graphical user interface presented cryptic moth targets that were super-imposed on tree photographs (as per Chapters 3 and 4). The computer monitor was rotated to a portrait orientation to show high resolution tree images (1600 x 800 pixels) with the moth target of $60 \times 100$ pixels in size. Computer screens were positioned $3 \mathrm{~m}$ away from subjects, making the visual angle of the target $0.35^{\circ}$ high by $0.73^{\circ}$ wide. Before the experiments commenced, several training screens were presented to individual subjects, exposing them to each treatment. Either one moth 
or no moth was presented per tree background during the search task. Subjects were instructed to click on the moth targets as fast as they could and that, once a target had been successfully discovered (or when the subject was satisfied that no target was present), they should proceed to the next screen by pressing the 'Release Moth' button. This allowed for a self-determined break period before the subjects resumed their visual search.

Sugar maple (Acer saccharum) trunks were photographed and thresholded at their median grayscale value and used to derive the targets. Thresholded areas were then coloured dark and light gray based on the original tree image's 25 th and 75 th grayscale percentiles using Adobe ${ }^{\circledR}$ Photoshop $®$. From these bi-coloured tree images, triangular areas were selected. The left half of the triangles were mirrored about the vertical axis of symmetry (Chapter 4). These bi-coloured triangles with vertical symmetry were designed to simulate moths.

Six target treatments were made using a $2 \times 2$ factorial design, with the factors being coloration (inside pattern; edge-interceptive pattern) and edge transparency (solid edge; transparent edge; see Fig. 5.1), with the addition of 2 plain controls. Targets with edge-intersecting patches were considered as putatively disruptively colored (sensu [15]). For each edge coloration treatment, ten unique base patterns were used. This withintreatment replication ensured that results were generalizable regarding the presence/absence of edge patches, whilst controlling for possible background matching differences between these treatments. Each of the base patterns was randomly sampled from the tree backgrounds. The constraint of ten replicates per coloration treatment was 
made to ensure that the experiment did not exceed 15 minutes in duration per subject (so as to maintain subject motivation during the computer foraging experiment).

Target boundary visibility was operationalized by increasing boundary transparency. Edge composition was varied by modifying solid edge targets and creating translucent boundaries (which had low boundary visibility). As the effect of translucent boundaries on object detectability has rarely been studied, I developed a protocol for deriving targets. Targets were created in Adobe Photoshop CS4 (Adobe Systems, Sans Jose, California) using the Lasso Tool to select an area of the size and shape of my triangle targets. This selection was super-imposed over a tree background and used for the control boundary treatment. To produce transparent edges, the selection's boundary is 'feathered' over 10 pixels (Select $>$ Modify $>$ Feather). Over this 10-pixel transect, opacity was incrementally decreased. As the result, the boundary then blends from a solid coloration to transparent at its periphery. This procedure did not change the size of the target. I concede that it is uncommon for Lepidoptera to have translucent boundaries. However, this makes moth-like target yet a further abstraction, allowing for the manipulation of boundary visibility for the purpose of testing a general prediction about the disruptive function of edge markings.

Using human predators allowed the flexibility to experimentally manipulate targets' transparency in ways that would not be possible in field experiments. It is difficult to produce targets that are both suitable for the field and have semi-opaque boundaries (edge composition was achieved using .png image files in the current study) because the material would need to have a similar refractive index to Lepidoptera's transparent structures (Binetti et al., 2009). Targets having dissimilar refractive indices 
would produce bright reflective glints in ambient lighting that would impair their capacity to background match (Johnsen, 2001). Computer foraging experiments are superior in this respect because they permit the testing of translucent boundaries without the challenges of light reflection.

Statistical analyses were performed in version 17 of SPSS (SPSS Inc., Chicago). Target detectability was measured as survivorship (proportion of each target type that went undiscovered) and search time (of those targets discovered, the time taken until found). Consequently, I utilized a multivariate general linear mixed model (GLMM), followed univariate analyses of the data. This stepwise approach allowed me to test if there was a consistent effect of the treatment variables for both survivorship and search time in one test, thereby minimising inflation of type I error rates. To derive survivorship from whether or not a target was discovered, I averaged over all subjects $(n=30)$. This yielded the proportion of targets missed for all tree backgrounds for each target type. Such post-processing allowed both response variables to share the same distribution in order to conduct the multivariate test. To conform to normality, search time was log transformed, and survivorship (a proportion) was arcsin transformed. The fitted models included tree background and targetID as random factors, and boundary visibility and edge coloration as fixed factors. To test my a priori prediction, I included a boundary visibility * edge coloration interaction term in the fitted model. 


\section{Boundary}

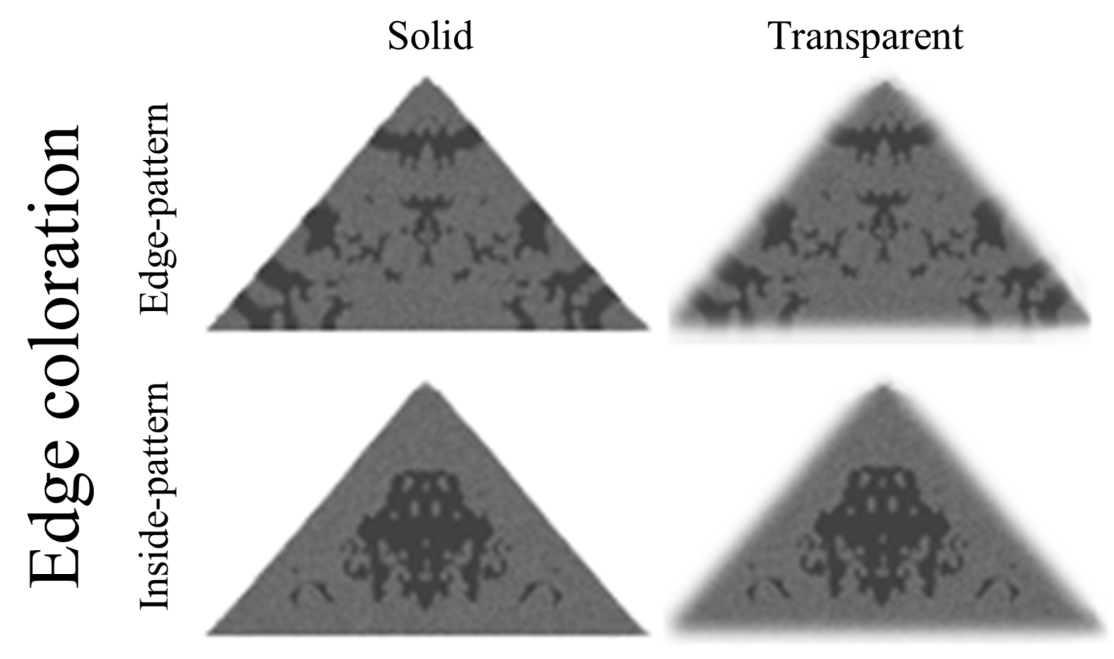

Figure 5.1: Illustration of the $2 \times 2$ factorial design of target treatments. 


\subsection{Results}

\subsubsection{Edge coloration}

There was a significant main effect of edge coloration treatment (presence / absence of edge markings) on moth target detectability (multivariate GLMM: Wilks' $\lambda_{2}$, ${ }_{553}=9.61, \mathrm{p} \leq 0.001 ;$ Table 5.1). Given this finding, univariate tests were performed and they showed consistently that target coloration affected target survival $\left(\mathrm{F}_{1,553}=17.89, \mathrm{p} \leq\right.$ 0.001; Table 5.2) and search time $\left(\mathrm{F}_{1,553}=12.08, \mathrm{p} \leq 0.001\right.$; Table 5.2). Therefore, the presence of edge-intersecting patches is associated with heightened survivorship and, for the discovered targets, a longer search time.

\subsubsection{Animal boundary visibility and concealment}

There was a significant main effect of boundary visibility (control \& translucent) on moth targets detectability using the multivariate GLMM (Wilks' $\lambda_{2,553}=6.35, \mathrm{p} \leq$ 0.001; Table 5.1). Given this finding univariate tests were performed, they showed consistently that the target's boundary effected survival $\left(F_{1,553}=9.47, p=0.002\right.$; Table 5.2) and search time $\left(\mathrm{F}_{1,553}=10.81, \mathrm{p} \leq 0.001\right.$; Table 5.2). Therefore, the presence of translucent target boundaries were associated with heightened survivorship and, of those targets discovered, a longer search time.

\subsubsection{Boundary visibility * edge coloration interaction}

There was a significant interaction of boundary * edge coloration on moth targets detectability (multivariate GLMM: Wilks' $\lambda$ 4, $1106=3.27, \mathrm{p} \leq 0.011$; Table 5.1). 
Given this finding, univariate tests were performed and they showed consistently that a target's boundary affected its survival $(F 2,553=5.30, p=0.005$; Table 5.2) and search time $(\mathrm{F} 2,553=3.49, \mathrm{p}=0.031$; Table 5.2). The combination of translucent boundaries and edge coloration thus interacted synergistically to enhance survivorship and, for those targets discovered, search time. 
Table 5.1: Factors affecting both survivorship and search time of moth targets 'hunted' by human subjects. The results of the multivariate general linear mixed model are presented. Here, Tree background and Pattern (target type) were controlled for in the model. Cells reporting results are formatted such as: Wilk's lambda test statistic, degrees of freedom, and p-value.

\section{Wilks' Lambda, df, p-values}

Transparency

Coloration

Tree

Pattern

Transparency*Coloration
$6.35, \quad 1, \quad 553, \quad \mathrm{p}<0.001$

9.61, $1, \quad 553, \quad \mathrm{p}<0.001$

5.17, 19 1106, $\mathrm{p}<0.001$

$4.28, \quad 19,1106, \quad \mathrm{p}<0.001$

$3.27, \quad 2, \quad 1106, \quad \mathrm{p}=0.011$ 
Table 5.2: Factors affecting survivorship and search time of moth targets 'hunted' by human subjects. The results of two univariate general linear mixed model are presented in separate columns with dependent variables as survivorship and searchtime. Tree background and Pattern (target type) were controlled for in the models. Cells reporting results are formatted as: F-test statistic, degrees of freedom, and p-value.

\begin{tabular}{llll}
\hline & \multicolumn{2}{c}{ Survivorship } & Search time \\
\hline Transparency & $9.47, \quad 1,553, \mathrm{p}=0.002$ & $10.81,1, \quad 554, \mathrm{p}<0.001$ \\
Coloration & $17.89,1,553, \mathrm{p}<0.001$ & $12.08,1,554, \mathrm{p}<0.001$ \\
Tree & $7.55, \quad 19,553, \mathrm{p}<0.001$ & $6.04,19,553, \mathrm{p}<0.001$ \\
Pattern & $7.33, \quad 19,553, \mathrm{p}<0.001$ & $5.26,19,553, \mathrm{p}<0.001$ \\
Transparency*Coloration & $5.30,2,553, \mathrm{p}=0.005$ & $3.49,2,553, \mathrm{p}=0.031$ \\
& & & \\
\hline
\end{tabular}


a.

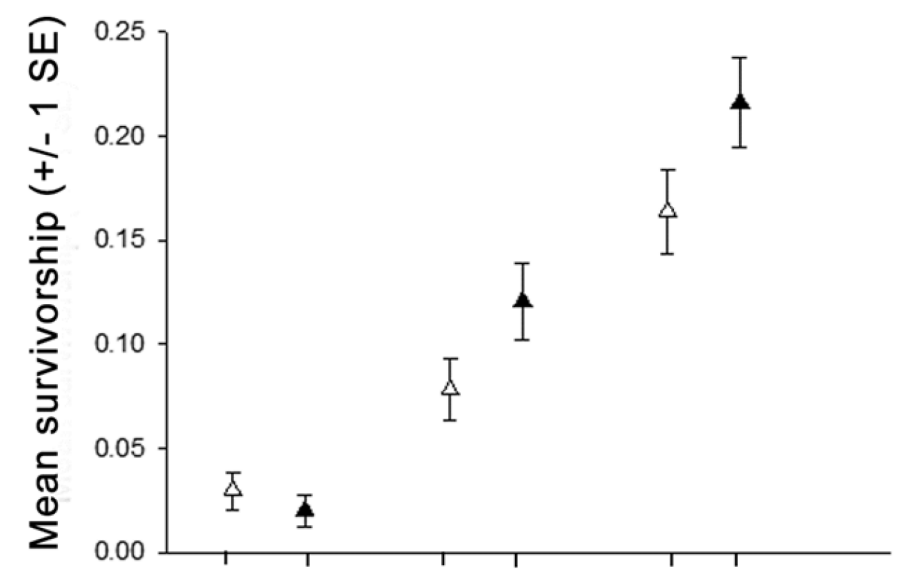

b.

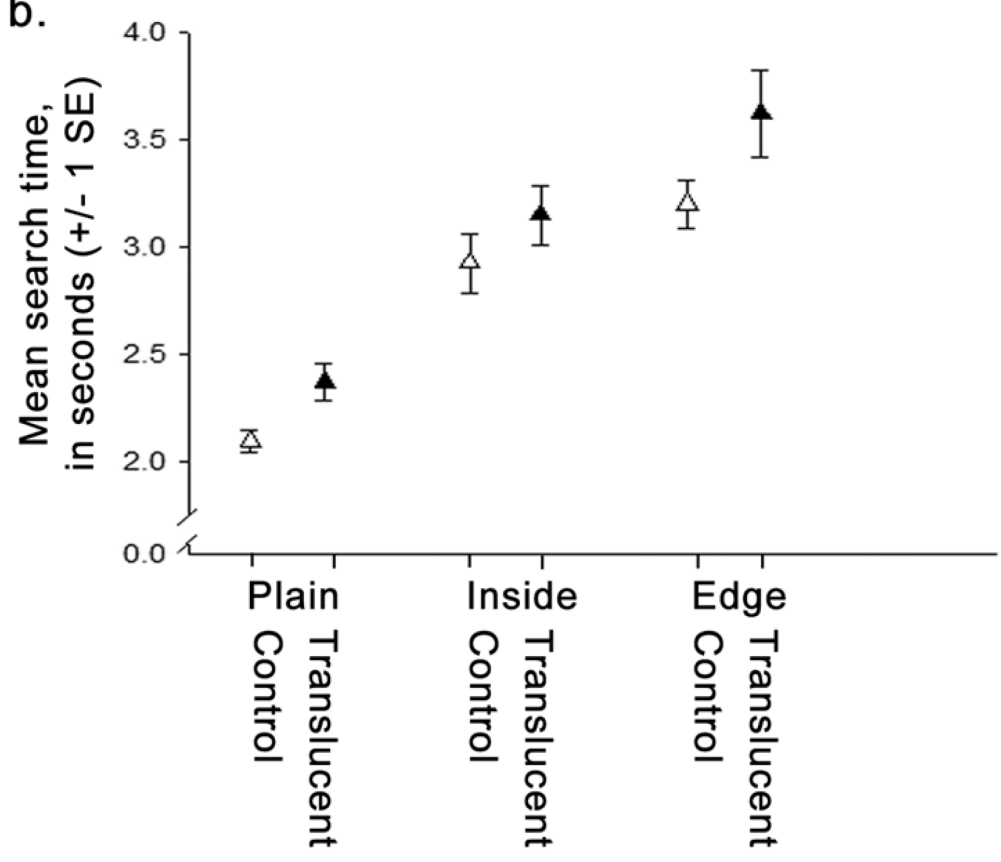

Figure 5.2: Detectability of moth targets that differed in boundary visibility and edge coloration. Edge intersecting patches increased (a) survivorship and $(b)$ search time. Closed triangle symbols denote targets with highly visible boundaries (control or normal with no transparency), and open triangles denote targets with low visibility boundaries (i.e. are translucent).Boundary visibility increased (a) survivorship and $(b)$ search time. The interaction effect, while statistically significant (Table 5.1 and Table 5.2), seems weak for $(a)$ survivorship, but more pronounced for $(b)$ search time. Targets with a combination of edge coloration with translucent boundaries are the most concealed. 


\subsection{Discussion}

The current study confirms previous work (Cuthill et al., 2005, Fraser et al., 2007, Merilaita and Lind, 2005, Stevens et al., 2006b, Schaefer and Stobbe, 2006, Cuthill et al., 2000, Cuthill and Szekely, 2009, Stevens et al., 2009, Chapter 4) demonstrating that the edge coloration of prey targets affects their detectability. The addition of edgeintersecting patches increased both survivorship and, for those targets that were discovered, time to detect. In the past, such a result would have been interpreted as direct evidence in support of the prediction that edge patches function to disrupt a target's outline and thereby impede its recognition. However, to directly test the functional nature of edge patches, it is necessary to manipulate features that are known to affect boundary perception, with the prediction that concealment due to edge markings is also dependent upon this other trait (see Fig 4.4). My experimental design here allowed me to do so by investigating whether the concealment value of edge patches is dependent on the visibility of the boundary.

To my knowledge, the current study is the first to investigate the effect of boundary visibility (mediated by transparency) on disruptive coloration. I used two treatments of boundary visibility (control and translucent). The translucent targets were ten translucent edge pixels deep, with the background visible through them. As expected, the boundary visibility treatment did affect target detectability, with the translucent boundary increasing both target survivorship and, for those targets that were discovered, time to detect. I speculate that translucent boundaries achieve camouflage by impeding edge detection. The translucent-boundary targets had low contrast with the background 
that they overlaid, due to colour fusion of their opaque boundary with the background (Beck et al., 1984). This lower contrast boundary is more challenging for edge detection (Geisler et al., 2001).

Multivariate analysis revealed a significant interaction between edge coloration and boundary visibility, which persisted in subsequent univariate tests. As with the findings reported in Chapter 4, the effects of edge coloration could be accounted for by a higher-order interaction term, which suggests that the benefit provided by edgeintersecting markings is dependent on the degree to which an outline is visible (i.e. the degree to which it contrasts against its background). This provides support for the proposition that edge-intersecting markings function as disruptive coloration because object concealment is conditional on boundary properties.

Edge coloration was most effective when combined with translucent boundaries. This synergistic interaction between edge coloration and translucent boundaries was surprising because it contradicts the results reported in Chapter 4. I am uncertain as to why this might be so. One possible explanation could be that both edge coloration and translucent boundaries affect an identical visual process (i.e. edge detection) and the process of breaking-up an outline has a non-linear effect on target recognisability, whilst shape perception is affecting a higher-order visual process. For instance, as the proportion of undetected edge changes from one quarter to one half, and then again from one half to three quarters, the rate at which recognisability will be degraded is slow at first but then rapidly increases. If both edge coloration and translucent boundaries affect a receiver's 
ability to detect edges, then it is plausible that these seemly separate target features could have a cumulative effect on outline break up; the outcome of which is a synergistic effect on target recognition and detectability.

The synergistic relationship discussed above is different from the masking interaction observed when edge coloration was combined with low-frequency ruffled shapes (Chapter 3). Reducing boundary visibility softens the otherwise abrupt transition between a target and its background, which functions to diminish the accuracy with which a predator can detect a target's edges (a low level step in the process of object recognition). In contrast, when a global shape deviates from simple, straight outlines, the overall appearance is dramatically altered (Wilson and Keil, 2001). Numerous, curvylinear boundaries function to increase a target's concealment because they are cognitively challenging to group into a whole (a mid/high level step in the process of object recognition). Interestingly, it seems that if a target has a complex shape it will have reduced detection rates (with or without edge-intersecting patches). Alternatively, decreasing a boundary's visibility (by increasing its transparency) appears to work synergistically with edge-intersecting patches. I speculate that edge-intersecting patches operate at a low level step in object recognition (edge detection); thus, when higher-order object recognition processes (recognition of an entire shape) are activated, the contribution of disruptive coloration on camouflage is could be less beneficial.

Alternatively, the nature of the interaction between edge colorations and other attributes that affect shape perception (i.e. shape / boundary visibility) could be 
dependent on whether the full extent to which shape recognition can be impaired has been maximized. It is reasonable to assume that there exists some finite limit for impairing shape recognition in a camouflage task. If edge coloration and translucent boundaries both provide small contributions to impairing shape perception, then their cumulative effect is synergistic. Conversely, when entire shape of a target is changed, there is a large effect on shape perception. It is possible that modification of target shape might reach the finite limit of impairing shape recognition and, therefore, a masking interaction with edge coloration will occur.

These findings have implications for combining disruptive markings with translucent objects. While translucent boundaries reduce the visibility of outlines, my findings suggest that the addition of disruptive markings will further enhance concealment of these boundaries. A group of animals known to use both transparency (Johnsen, 2001) and edge markings (Barbosa et al., 2008) strategies are cephalopods. The predation risk faced by cephlapods in different light conditions has led to their evolving a dynamic strategy to optimize camouflage. The cephlapods Japetella heathiif and Onychoteuthis banksii switch between defensive camouflage strategies (Zylinski and Johnsen, 2011), using transparency in ambient light, to pigmentation (putatively disruptive edge markings) during bioluminescent searchlight activity of their predators. Such cephlapod pigmentation absorbs the red-green searchlights produced by their predators to better match their dark background. Cephlapods are also known to express contrasting 'disruptive' markings depending on environmental conditions (Barbosa et al., 
2008, Chiao et al., 2005, Chiao et al., 2013). Future work should test if cuttlefish combine their disruptive coloration and transparency to enhance camouflage.

For the field of biotechnology, these findings are timely with respect to recent developments on camouflage through invisibility. There has been several advances in nano-scale invisibility cloaks (Valentine et al., 2009, Chen et al., 2010, Chen and Alù, 2011) (where at the nano level particles can have light bent around them, creating the impression of a invisibility cloak). Another development includes the announcement of an invisibility cloak by Hyper Stealth ${ }^{8}$ (Vancouver, Canada) (Cramer, 2013). An implication of the current study for these developments is that translucent edges can achieve additional concealment benefits if combined with disruptive camouflage.

To test if a colour marking is disruptive (sensu stricto), it is necessary to test predictions of how disruptive coloration functions. Previously, the occurrence of edge coloration has been correlated with lower detection rates in receivers, which provided researchers with indirect evidence for disruptive coloration. Here, I demonstrate experimentally that edge coloration and boundary visibility interact to enhance disruptive camouflage, thereby providing more direct evidence that putatively disruptive markings function independently from, and are not a by-product of, other camouflage strategies. Further, this research provides insight into the mechanisms that explain how disruption achieves concealment.

\footnotetext{
${ }^{8}$ Hyper Stealth is a Canadian company that developed CADPAT's Urban equivalent (CUEPAT) and hold many large contracts in NATO countries.
} 


\section{Chapter 6: Disruptive camouflage impairs object recognition}

"The function of a disruptive pattern is to prevent, or to delay as long as possible, the first recognition of an object by sight." (Cott, 1940)

\subsection{Abstract}

Whether hiding from predators, or avoiding battlefield casualties, camouflage is widely employed to prevent detection. Disruptive coloration is a seemingly well-known camouflage mechanism proposed to function by breaking up an object's salient features (such as their characteristic outline), rendering objects more difficult to recognise. However, while a wide range of animals are thought to evade detection using disruptive patterns, there is no direct experimental evidence that disruptive coloration impairs recognition. Using humans searching for computer-generated moth targets, I demonstrate that the number of edge-intersecting patches on a target reduces the likelihood of it being detected, even at the expense of reduced background matching. Crucially, eye tracking data show that targets with more edge-intersecting patches were looked at for longer periods prior to attack, and passed-over more frequently during search tasks. I therefore show directly that edge patches enhance survivorship by impairing recognition, confirming that disruptive coloration is a distinct camouflage strategy, not simply an artefact of background matching.

This chapter has been submitted for publication: WEBSTER, R.J., HASSALL, C., HERDMAN, C.M., GODIN, J-G.J \& SHERRATT, T.N. 2013. Disruptive camouflage impairs object recognition. Biology Letters 9 (6) 10.1098/rsbl.2013.0501. 


\subsection{Introduction}

Camouflage is a ubiquitous phenomenon in nature, with natural selection favouring animals that can avoid being seen and recognised by predators (Thayer, 1909, Cott, 1940, Ruxton et al., 2004b). The most intuitive forms of camouflage are based on blending in with the background (background matching) and on mimicking an irrelevant object in the environment (masquerade). However, camouflage is also thought to be achieved in a wide range of species via disruptive coloration. Disruptive coloration functions by obscuring outlines and creating false boundaries, thereby preventing recognition of an animal's salient features (Thayer, 1909, Cott, 1940, Ruxton et al., 2004b, Troscianko et al., 2009). While the concept of disruptive coloration has been standard textbook material for over a century (Thayer, 1909), with applications ranging from military uniforms and equipment to art (Behrens, 2002), empirical verification of the functional mechanism underlying disruptive coloration has remained elusive for two main reasons: first, disruptive coloration invariably occurs in conjunction with background matching, so disentangling their individual contributions to camouflage is therefore challenging (Silberglied et al., 1980, Stevens, 2007, Stevens and Merilaita, 2009). Second, disruptive coloration is best defined in terms of its function (impairing recognition) rather than its appearance (Stevens 2007; Stevens and Merilaita 2009). Consequently, despite repeated suggestions that animals from anteaters to zebra are disruptively coloured, one cannot, based on appearance alone, state that an animal's body pattern is disruptive. Cuthill et al. (2005) provided the first field demonstration that artificial prey targets with contrastingly patterned edges ('Edge' targets) had a greater survivorship under bird predation than control targets without edge-intersecting patches. Similar results were subsequently 
reported with human subjects visually hunting for artificial moth targets displayed on computer screens (Fraser et al., 2007), wild birds foraging in aviaries (Merilaita and Lind, 2005) and wild birds foraging in their natural habitats (Stevens et al., 2006b, Schaefer and Stobbe, 2006, Cuthill et al., 2000, Cuthill and Szekely, 2009, Stevens et al., 2009). Unfortunately, however, there is no experimental evidence to confirm that the enhanced survivorship afforded by such markings was attained through impaired object recognition (Cuthill and Troscianko, 2011). Put simply, results showing that edge-intersecting patches enhance survivorship are necessary, but not sufficient. For disruptive coloration to work in the manner prescribed, then object recognition must be impaired, otherwise the theory fails (Cuthill and Troscianko, 2011).

\subsection{Methods}

Using eye-tracking technology, I test, for the first time, if edge-intersecting patches impair object recognition and thereby enhance their survivorship. Eye-tracking is widely used in psychophysics (Duchowski, 2002) and is particularly well suited to distinguish failure of object recognition from a failure to locate a hidden target ((Credidio et al., 2012, Johnson et al., 2003); Fig. 6.1). The eye movements of individual subjects were quantified during each search task using a FaceLab ${ }^{\mathrm{TM}}$ eye tracker (Seeing Machine, Canberra, Australia) which recorded foveal (line of sight) eye positions at a frequency of $60 \mathrm{~Hz}$. Raw eye movement data included both fixations and saccades. Due to limited visual information gained during the latter, a velocity-base threshold (see ESM-Tracking of eye movements) was used to identify and exclude saccarde data from subsequent analyses. Analysis of the eye-tracking data allowed me to quantify three inter-related measures of object recognition, namely (i) inspection time (the total time that each 
subject's spent foveating within $1.5^{\circ}$ of the target), (ii) number of passes (independent bouts during which foveal vision moved from outside to inside the $1.5^{\circ}$ zone around the target, and is therefore-for passes $>1$-a measure of false negative rate for object recognition; see Fig. 6.2 \& Appendix Fig. E.1), and (iii) final inspection time (the time spent foveating within $1.5^{\circ}$ of the target, in the bout immediately prior to attacking). If disruptive coloration hinders recognition of a target through breaking up a target's outline, then targets with more disruptive color patterns should be inspected for longer periods and incur more frequent independent passes from the searcher. Moreover, variation in the above measures should explain some of the observed variation in target survival and total search time.

I monitored the eye movements of 48 human subjects hunting for 63 artificial, bicolored triangular moth targets with different coloration patterns placed on tree-trunk backgrounds. The trees and moths were displayed on a computer screen using a Visual Basic 8 GUI, which also recorded (i) if targets were discovered, and (ii) total visual search time (in milliseconds) of the subjects for the discovered targets. When a subject recognised the target, he/she immediately rotated the mouse wheel to stop the timer and then moved the cursor over the target and clicked on it to verify that the target had been correctly located. For each of the 63 moth targets, I quantified three of its inter-related coloration properties (Appendix Fig. E.2 for a gallery illustrating variation), namely, uniformity (the overall heterogeneity of markings, with high values representing highly mottled appearance), the proportion of dark area in the bicoloured targets, and the number of edge-intersecting patches (the number of groups of five or more continuous dark pixels that touch the targets outline, used as my measure my putative 'disruptive' coloration, as more edge patches contribute to the breakup of target's outline (Stevens and Cuthill, 2006, Chen He and Yung, 2008; Appendix Fig. E.3). 


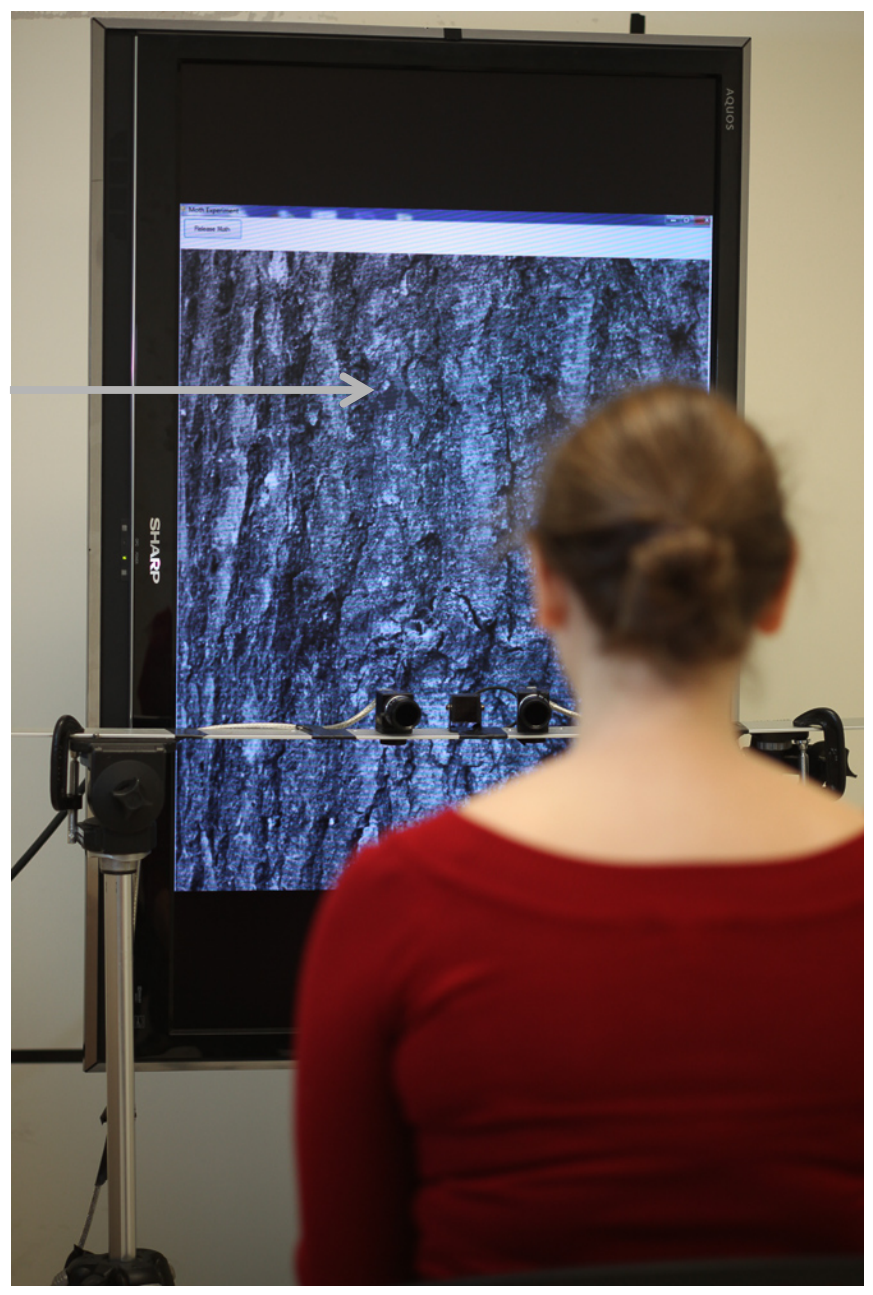

Figure 6.1: Eye tracking human subjects and experimental protocol. Measuring recognition is challenging, but studying the eye movements of human subjects in a lab setting provides a tractable system to observe object recognition of camouflaged targets. The human subjects used in the current study were presented with a portrait screen displaying an image of a tree-trunk, onto which a cryptic moth target (at arrow tip) was randomly located. Subjects were seated $1.8 \mathrm{~m}$ away from and facing the screen and were given a brief training period on a set of moth targets unique from the experimental targets in colour pattern, but identical in shape and size. A narrow metal beam, mounted on two tripods, held in place the stereo cameras and IR pod which were directed at the subject's eyes. 


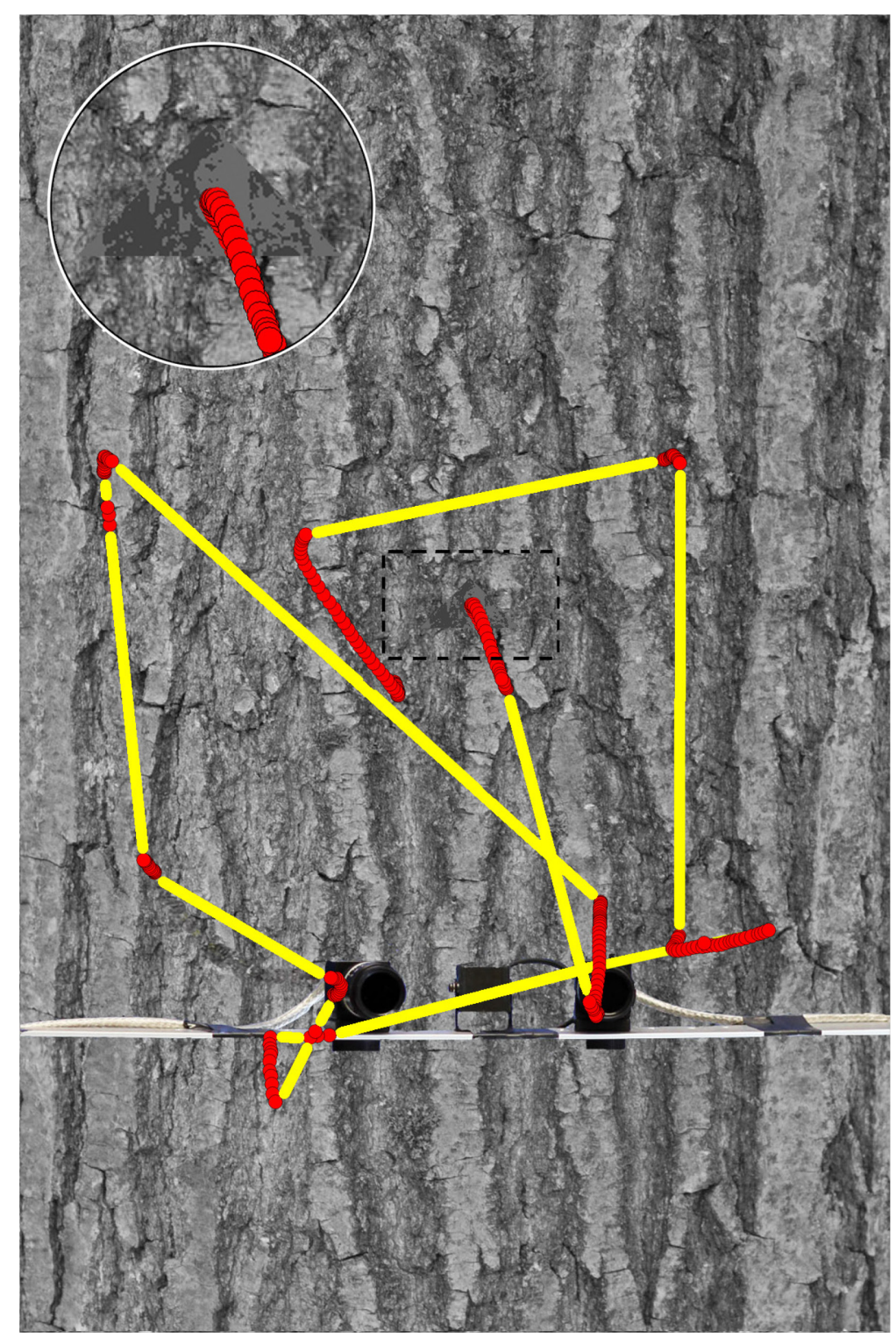

Figure 6.2: Camouflaged moth-like target on tree background, superimposed with eye tracking data. Here, the red dots indicate the location of the subject's gaze on the screen (one sampling point every $17 \mathrm{~ms}$ ), with the yellow line interpolating between consecutive recordings to draw a visual search path. The black dashed square highlights the zone of interest where foveal eye movement implies spatial attention directed to the target (see ESM, Tracking of eye movement). An increase in total time spent foveating or number of independent visits to the zone of interest ("passes") indicates — indirect and directly — that the recognition task for a given target is more challenging. 


\subsection{Results}

Overall, the 63 moth targets differed in their survivorship (i.e. the proportion of each target that went undiscovered over all presentations; Fig. $6.3 b$ for examples of high and low survivorship targets; $\chi_{62}^{2}=90.1, p<0.05$; range $=0-18 \%$ ) and mean search time for those found (i.e. total time taken to discover each target type; $\mathrm{F}_{62,2544}=5.05, p<0.001$; means per target $2.5-15.8 \mathrm{~s}$; Fig. $6.3 c$ ). If edge-intersecting patches disrupt object recognition, then the survivorship and mean search time of targets should increase with increasing number of edge-intersecting patches, while controlling for other overall aspects of target appearance (in this case uniformity and proportion of dark area). As predicted, as the number of edge patches increased, so did average survivorship of the target $\left(\mathrm{F}_{1,59}=4.25, p=0.044\right.$; Table 6.1, Fig. 6.1b), as well as the mean search time for those discovered $\left(\mathrm{F}_{1,59}=8.36, \mathrm{p}=0.005\right.$; Table 6.1, Fig. $\left.6.3 \mathrm{c}\right)$, over and above the effects of the uniformity and darkness of the targets.

Targets with more edge-intersecting patches were more difficult to recognise by human subjects. All three recognition metrics, total inspection time $\left(\mathrm{F}_{1,59}=18.83, p<\right.$ 0.001, Fig. 6.4a), final inspection time $\left(\mathrm{F}_{1,59}=9.34, p=0.003\right.$, Fig. $\left.6.4 \mathrm{~b}\right)$ and the number of passes $\left(\mathrm{F}_{1,59}=7.03, p=0.010\right.$, Fig. $\left.6.4 \mathrm{c}\right)$ significantly increased as the number of edge-intersecting patches increased, while controlling for other aspects of the targets' appearance. Targets with more edge-intersecting patches were more difficult to recognize, despite those targets with a high number of edge patches being less representative of their background (Appendix Fig. E.4-E.6). The number of edgeintersecting patches was not the only target characteristic to affect recognition. Of the 
background matching metrics used, uniformity also affected the number of passes, whereas target darkness had no effect on any recognition metric (Table 6.1). Lastly, I note that inspection time itself was a significant contributor to total search time $\left(\mathrm{F}_{1,57}=\right.$ 5.85, $p=0.019$, Appendix Fig. E.7 and Appendix Table E.4), while the number of edge patches interacted with inspection time to affect overall search time $\left(\mathrm{F}_{1,57}=12.28, p=\right.$ 0.001; Appendix Table E.4). 

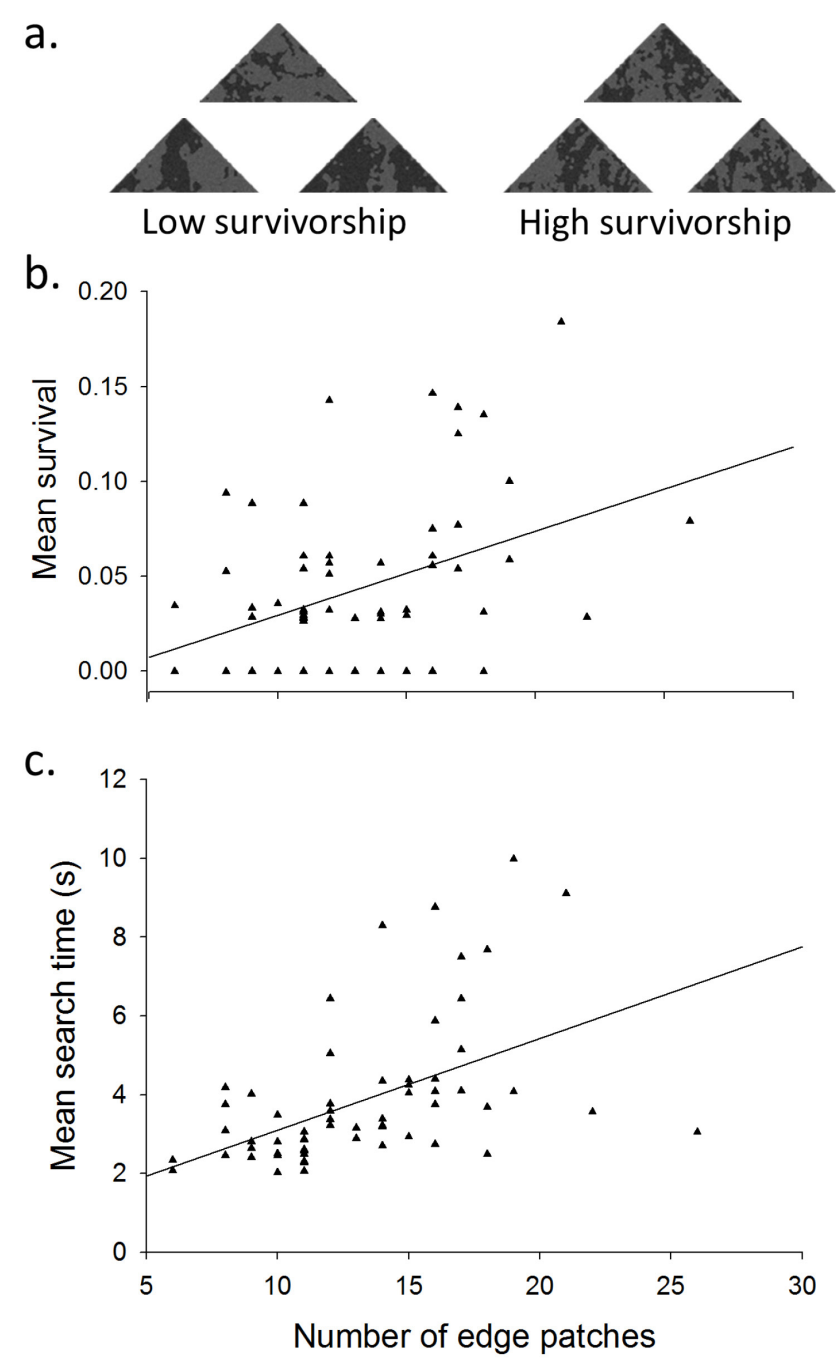

Figure 6.3: Detection probability of moth targets as a function of edge properties. (a) Examples of moth targets with low and high survival over all trials. Targets with a high number of edge-intersecting patches tended to have $(b)$ higher mean survival, and $(c)$ higher mean search time (of those targets discovered). Each data point represents a target, with individual mean survivorship and search time calculated over 48 independent subject trials. Lines represent fitted least-squares regression, estimated from the fits of general linear models in Table 6.1. 


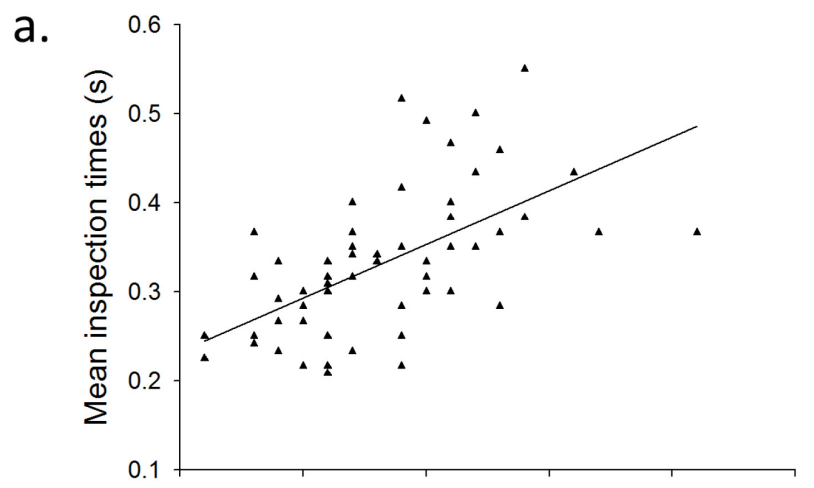

b.
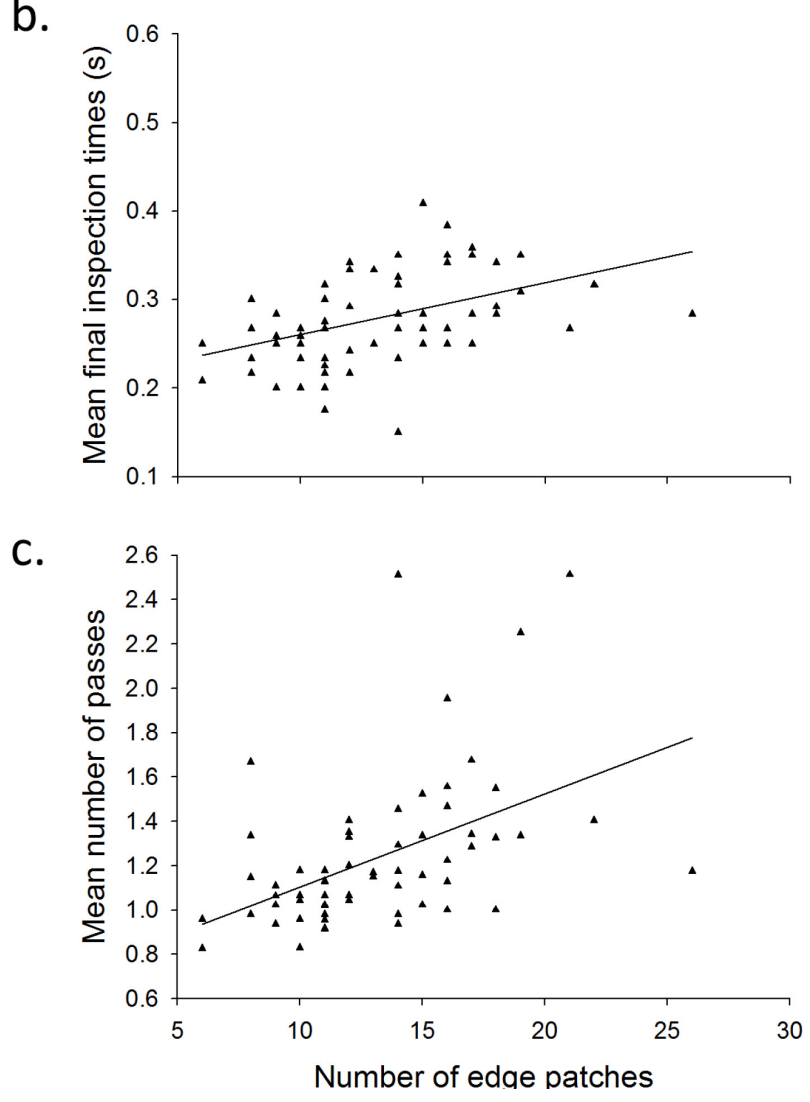

Figure 6.4: As the number of edge-intersecting patches increase, the recognition of moth targets becomes harder. When visually hunted by human subjects, moth targets with more edge-intersecting patches tend to have (a) a higher mean inspection time, (b) a higher mean final inspection time, and (c) a higher mean number of passes. Lines represent fitted least-squares regression, estimated from the fits of general linear models in Table 6.1. 
Table 6.1. Testing for the effects of the coloration properties of moth targets on their detection and recognition by human subjects.

Each data column shows the results of the fit of a separate general linear model. All fitted models included three target coloration properties as predictor variables (number of edge-intersecting patches, total proportion of targets dark area, and uniformity). These predictors were fitted using Type III sums of squares to control for the effect of correlated coloration properties. The geometric means for the detectability and recognisability of each target were calculated across all 63 target presentations. Test statistics reported for the fitted models are $F$ values ( $d f=1,59$ in all cases), with significance denoted by $* p<0.05, * * p<0.01, * * * p<0.001$.

\begin{tabular}{|c|c|c|c|c|c|c|}
\hline \multirow{3}{*}{\multicolumn{2}{|c|}{\begin{tabular}{cc}
\multicolumn{2}{c}{ predictor variables } \\
type of \\
coloration
\end{tabular}$\quad$ target coloration variable }} & \multicolumn{5}{|c|}{ dependent variables } \\
\hline & & \multicolumn{2}{|c|}{ detectability } & \multirow{2}{*}{\multicolumn{2}{|c|}{$\begin{array}{l}\text { recognisability } \\
\text { final } \\
\text { inspection time }\end{array}$}} & \multirow[b]{2}{*}{ number of passes } \\
\hline & & $\begin{array}{c}\text { survival } \\
\text { (log transformed) }\end{array}$ & $\begin{array}{c}\text { search time } \\
\text { (log transformed) }\end{array}$ & & & \\
\hline Edge & edge-intersecting patches & $4.26, p=0.044 *$ & $8.36, p=0.005^{* *}$ & $18.83, p<0.001 * * *$ & $9.34, p=0.003 * *$ & $7.03, p=0.010^{* *}$ \\
\hline Overall & total prop. of dark area & $0.12, p=0.729$ & $0.87, p=0.360$ & $0.08, p=0.782$ & $0.135, p=0.714$ & $0.15, p=0.700$ \\
\hline Overall & uniformity & $3.99, p=0.050 *$ & $7.20, p=0.009 * *$ & $1.401, p=0.241$ & $0.06, p=0.9390$ & $4.48, p=0.039 *$ \\
\hline
\end{tabular}




\subsection{Discussion}

Target types with a high number of edge patches survived better and took longer to be discovered, even when they were less representative of the backgrounds (Merilaita, 1998; Appendix Fig. E.7); therefore, the observed effect of number of edge-intersecting patches could not have arisen as a consequence of targets with higher number of edge patches being better background matchers. Of the two measures of overall target coloration pattern, lower uniformity (a more mottled appearance) was associated with significantly increased survival and search time, whereas proportion of dark area was not (Table 6.1). My central finding, that edge-intersecting patches increase survival and search time, remained valid when a more detailed model was fitted to encompass individual trials, with human subject as a random factor (Appendix Table E.2 \& E.3). The observed endpoints are precisely what one would expect if disruption mediates the contribution of object recognition to overall detectability. Intriguingly, inspection time represents a relatively small fraction of total search time, and yet small changes to inspection time have a large effect on total search time (Appendix Fig. E.7). This outcome may have arisen as a consequence of subjects being reluctant to return to an area that they had previously searched (Klein, 2000). Alternatively or in addition, the low proportion of inspection time to total search time could miss out other processes that allow object recognition, as my measures exclude the role of the subjects' peripheral vision (Appendix E.2).

Collectively, my results provide the first direct evidence that a target's edge patterning hinders object recognition, supporting the view that disruptive coloration can 
be regarded as a form of camouflage that is functionally distinct (Stevens 2007; Stevens and Merilaita 2009) from background matching. While previous studies have demonstrated that targets with edge markings have higher survivorship (Cuthill et al., 2005, Fraser et al., 2007, Merilaita and Lind, 2005, Stevens et al., 2006b, Schaefer and Stobbe, 2006, Cuthill et al., 2000, Cuthill and Szekely, 2009, Stevens et al., 2009), their unmanipulated 'disruptive' treatments could conceivably have been more representative of the backgrounds on which they are presented. I have overcome this limitation in the current study, not only by statistically controlling for obvious forms of background matching but also by showing that objects with a high number of edge patches, less representative of their backgrounds, tend to survive at higher rates because they are more difficult to recognize. Only through accounting for how disruption functions in this manner can I understand precisely why so many animal species have evolved highcontrast body markings (Caro, 2009) and evaluate what has been dubbed "the most important set of principles relating to concealment" (Cott, 1940). 


\section{Chapter 7: General Discussion and Conclusions}

In this closing chapter, I discuss some of the wider implications of this dissertation's findings within the context of the function and evolution of animal camouflage. Here, I go beyond highlighting the key findings of each chapter and indulge in some conjecture.

\subsection{Disruptive coloration}

It is challenging to separate the effects of disruptive coloration on concealment from those of background matching since, while disruptive coloration has a discrete camouflage function, it likely works in tandem with background matching. Although disruptive coloration is proposed to be a widespread phenomenon in nature, there is limited empirical work to characterize it. The few studies that do exist often ascribe disruptive coloration to animal body markings that visually resemble putative 'disruptive' coloration or from putative 'disruptive' markings associations with enhanced survivorship (Cuthill et al., 2006a, Cuthill et al., 2005, Cuthill et al., 2000, Stevens et al., 2006b, Stevens et al., 2009, Schaefer and Stobbe, 2006, Dimitrova et al., 2009, Merilaita and Lind, 2005). There are two issues with this: (1) this is a subjective approach that uses visual appearance to identify an animal coloration strategy that has a functional definition (and should therefore be identified by function, not appearance), and (2) the higher survivorship associated with putative 'disruptive' markings could be a by-product of another camouflage strategy. 
This thesis adopted several approaches to disentangle disruptive coloration from background matching. First, in Chapter 6, a Type III sums-of-squares analysis was used to control for possible collinearity between number of edge-intersecting patches and possible confounding background matching coloration (Appendix Fig. E.8). Second, I quantified the relative abundance of edge patches to show that colorations least representative of the background had the highest survivorship, an approach extended from (Merilaita, 1998). Finally, the relation between of number of edge-intersecting patches and survivorship was linear, as opposed to a quadratic function expected from optimal background matching. Taken together, I provide both indirect and direct ways to separate disruptive coloration from background matching.

Throughout my thesis, I have argued that evidence of underlying disruptive function is required for disruptive coloration to be considered as distinct from background matching. For instance, several studies have already shown greater survivorship for camouflaged targets (Cuthill et al., 2005, Cuthill et al., 2006b, Merilaita and Lind, 2005, Stevens et al., 2006b, Fraser et al., 2007). However, this thesis explores how these edge-intersecting patches function to achieve reduced detection rates by testing the following predictions: (i) edge coloration increases concealment that is dependent on visibility of shape and boundary properties, (ii) edge coloration breaks up an animal's outline, and (iii) edge coloration impairs object recognition. Such predictions are much more conservative (than if just controlling for background matching and looking for survivorship differences) since they are tailored to verify if the coloration behaves according to the how disruptive marks operate (Stevens and Merilaita, 2009), as opposed 
to only based on having a similar outcome (reduced detection rates). Testing predictions about the how disruption functions is clearly more demanding than the evidence is required to diagnose potential disruptive coloration at work.

In concordance with previous work, this thesis showed that edge coloration increases survivorship. In addition, it provides novel insight that edge coloration camouflage is dependent upon the characteristics of shape (Chapter 4; Fig. 4.4) and boundary (Chapter 5; Fig. 5.2) visibility (Table 7.1). By varying the shape or boundary of a target, one is able to investigate how edge coloration functions to increase survivorship. An interaction between edge coloration and factors known to affect shape and boundary perception would suggest that enhanced concealment provided by edge coloration is conditional on properties that disruptive coloration is predicted to affect (Fig. 4.2). Conversely, the absence of an interaction term would suggest that edge coloration acts through background matching, as it is independent of properties of shape and boundary. It would seem that edge coloration facilitates concealment by making targets appear to have a complex shape. Interestingly, the nature of these interactions is different: whilst some speculations are made as to why this might be (i.e. due to a finite capacity to benefit from impairing shape perception), more work is needed to investigate the discrepancies.

To further study how edge coloration provides camouflage, I investigated the longstanding assumption that disruption impairs object recognition. I monitored the behaviour of foveal vision of human subjects using eye-tracking technology and found that targets with more edge-intersecting patches break-up target outlines (Appendix Fig. 
E.3). Further, targets with more edge-intersecting patches are inspected for longer periods of time (Fig. 6.4a) and are overlooked more often (false negative; Fig. 6.4b) than targets with fewer edge patches. Interestingly, as the number of edge-intersecting patches increases beyond a critical point, this coloration becomes progressively less representative of the background (Appendix Fig. E.4) and yet edge patches continue to render target recognition more difficult (Appendix Fig. E.6). Such impaired recognition facilitates disruptive coloration at the expense of background matching. This observation explains previous empirical findings that disruptive coloration contributes to concealment independent of background matching (Fraser et al., 2007, Schaefer and Stobbe, 2006), through hindering object recognition (and not by resembling background properties). Consequently, animals with disruptive coloration change predator-prey encounters (Lima and Dill, 1990); even if animals with disruptive coloration are seen, they may be misidentified repeatedly, if not indefinitely. Taken together, these findings address Thayer's century-old question that disruptive coloration is a distinct form of camouflage, functioning to break up a target's outline and shape, by impairing object recognition. 
Table 7.1: An extensive summary of empirical work done on disruptive coloration. Columns denote the types of evidence test, with the symbols representing whether the predictions of disruptive coloration were $(\checkmark)$ or were not $(\times)$ satisfied. Note that the last several rows show the novel contribution that this thesis has made to the theory of disruptive coloration.

\begin{tabular}{|c|c|c|c|c|c|c|c|c|}
\hline \multicolumn{2}{|c|}{ Study information } & \multicolumn{4}{|c|}{ Evidence for disruptive coloration } & \multicolumn{3}{|c|}{$\begin{array}{l}\text { Functional evidence for } \\
\text { disruptive coloration }\end{array}$} \\
\hline Study name & $\begin{array}{l}\text { Laboratory } \\
\text { (L), Field } \\
\text { (F) or } \\
\text { Human exp. } \\
\text { (H) }\end{array}$ & $\begin{array}{l}\text { Cryptic } \\
\text { coloration un- } \\
\text { representative } \\
\text { of the } \\
\text { background }\end{array}$ & $\begin{array}{c}\text { Visual } \\
\text { appearance } \\
\text { associated } \\
\text { with } \\
\text { heightened } \\
\text { survivorship }\end{array}$ & $\begin{array}{c}\text { Independent } \\
\text { of } \\
\text { background } \\
\text { matching }\end{array}$ & $\begin{array}{c}\text { Reliant on } \\
\text { background } \\
\text { matching }\end{array}$ & $\begin{array}{c}\text { Dependent } \\
\text { on shape I } \\
\text { boundary } \\
\text { visibility }\end{array}$ & $\begin{array}{c}\text { Effects } \\
\text { edge } \\
\text { detection }\end{array}$ & $\begin{array}{l}\text { Impairs } \\
\text { recognition }\end{array}$ \\
\hline Silberglied et al. (1980) & $\mathrm{F}$ & & $x$ & & & & & \\
\hline Merilaita (1998) & $\mathrm{F}$ & $\checkmark$ & & & & & & \\
\hline Cuthill et al. (2005) & $\mathrm{F}$ & & $\checkmark$ & & & & & \\
\hline Chiao et al. (2005) & F L & & & & $\checkmark$ & & & \\
\hline Merilaita \& Lind (2005) & $\mathrm{F}$ & & $\checkmark$ & & & & & \\
\hline Stevens \& Cuthill (2006) & $\mathrm{F}$ & & & & & & $\checkmark$ & \\
\hline Cuthill et al. (2006) & $\mathrm{F}$ & & $\checkmark$ & & & & & \\
\hline Schaefer \& Stobbe (2006) & $\mathrm{F}$ & & $\checkmark$ & $\checkmark$ & & & & \\
\hline Stevens et al. (2006) & $\mathrm{F}$ & & $\checkmark$ & & & & & \\
\hline Fraser et al. (2007) & $\mathrm{H}$ & & $\checkmark$ & & $\checkmark$ & & & \\
\hline Cuthihll \& Szekely (2009) & $\mathrm{F}$ & & $\checkmark$ & & & & & \\
\hline Dimitrova \& Merilaita (2009) & $\mathrm{F}$ & & $x$ & & & & & \\
\hline Caro (2013) & $\mathrm{F}$ & & $x$ & & & & & \\
\hline Thesis Chapter 4 & $\mathrm{H}$ & & $\checkmark$ & & & $\checkmark$ & & \\
\hline Thesis Chapter 5 & $\mathrm{H}$ & & $\checkmark$ & & & $\checkmark$ & & \\
\hline Thesis Chapter 6 & $\mathrm{H}$ & $\checkmark$ & $\checkmark$ & $\checkmark$ & & & $\checkmark$ & $\checkmark$ \\
\hline
\end{tabular}




\subsection{Background matching}

In testing for disruptive coloration, this thesis has also contributed to enhancing our understanding of background matching. Chapter 6 showed that artificial moths that are darker and/or have a highly mottled coloration (viz. uniformity) coloration have lower detectability on greyscale sugar maple tree trunk backgrounds. This finding corroborates those of past studies that have demonstrated that animals achieve background matching through both darkness (Vignieri et al., 2010, Kats and Vandragt, 1986, Chang et al., 2012, Cox et al., 2009) and mottled coloration (Chiao et al., 2010). However, care should be taken in generalising these results to all moth species that camouflage themselves on trees, since the experimental paradigm of positioning moths randomly on trees doesn't allow for realistic moth behaviours such as orientation (Chapter 2), body posture (Barbosa et al., 2012) and micro-patch choice (Kang et al., 2012). It is possible that, if such behaviours were included, different background matching properties might affect moth concealment.

A fundamental principle of camouflage is its dependency on background properties. Based on the psychology and vision literatures, the question of 'on which background are objects more difficult to detect?' is already an established research area. Unfortunately, this research often offers limited interpretation of background matching in the context of animal camouflage, because the backgrounds typically used are very simple (e.g. (Boot et al., 2009)) and lack the dimensions of natural scenes that play a critical role during visual search tasks (i.e. texture (Nyberg and Bohman, 2001, Elder and Velisavljevic, 2009), directionality (Geisler et al., 2001, Coppola et al., 1998), fourier 
power spectrum (Girard and Koenig-Robert, 2011), range of colour (Liebe et al., 2009), luminance (Frazor and Geisler, 2006, Ludwig and Gilchrist, 2006) and high contrast (Bex and Makous, 2002, Frazor and Geisler, 2006, Macé et al., 2010)). Even in the study of animal camouflage, this limitation of visually simplistic background can be found (Dimitrova and Merilaita, 2012, Dimitrova and Merilaita, 2009, Dimitrova and Merilaita, 2010). Previous theoretical work on animal camouflage has suggested that increasing the complexity of backgrounds also increases the difficulty of detecting animals present on them (Merilaita, 2003), a prediction that has been supported by empirical studies in which the diversity of background visual elements was varied (Dimitrova and Merilaita, 2012, Dimitrova and Merilaita, 2009, Dimitrova and Merilaita, 2010, Kjernsmo and Merilaita, 2012). An important strength of my thesis research is that I use grayscale photographs of tree trunks as backgrounds, which are complex backgrounds. Interestingly, in all data chapters of this thesis, the variable 'tree specimen' was included as a random factor in the analyses and substantially contributed to target detection. Since I only wished to control for the between-tree specimen differences here, background characteristics of trees that might have contributed to detectability differences between targets were not investigated; however, this issue represents an intriguing future direction for investigation. A broader question that emerges from this is: to what degree of abstraction can concepts of animal camouflage be tested, whilst retaining ecological validity? 


\subsection{Combining camouflage strategies}

A commonly held principle in camouflage is that concealment is compromised as animals generalize their coloration to blend into multiple, visually-distinct habitats (Merilaita et al., 1999, Houston et al., 2007, Ruxton et al., 2004a). It is known that the efficacity of background matching in animals is reduced as its fidelity to the ambient environment diminishes. However, it is possible that other camouflage strategies can provide concealment without compromising their efficacy between visually-distinct backgrounds. Disruptive coloration has been proposed to extend camouflage in a way that minimizes this 'multiple-backgrounds' compromise (Thayer, 1909, Merilaita et al., 1999, Sherratt et al., 2005, Schaefer and Stobbe, 2006). Since disruptive camouflage is semi-independent of background, the addition of disruptive coloration to moderately background-matched targets would likely improve overall camouflage. It is thus predicted that habitat generalists might rely more on disruptive coloration than habitat specialists (Merilaita et al., 1999) (as generalists' range would occupy a greater diversity of visually-distinct backgrounds); this prediction remains untested. If this prediction is correct, disruptively coloured species could benefit from an extended range of habitats, allowing them to take advantage of increased access to food, mating opportunities, etc., without increased predation risk. Several studies, including this thesis (Chapter 6), have shown that disruptive colour patterns can be dissimilar to their background and still facilitate concealment (Fraser et al., 2007, Schaefer and Stobbe, 2006). However, at some critical threshold, a sufficient lack of background matching results in disruptive coloration no longer providing concealment (Fraser et al., 2007, Stevens et al., 2013, Stevens et al., 2006b, Stobbe and Schaefer, 2008). More work is thus needed to investigate how the 
mutual camouflage strategies of disruptive coloration and background matching are combined, especially when attempting to attain optimal camouflage solutions for habitat generalists.

Background matching and disruptive coloration can also be combined with colour polymorphism. Polymorphism has been shown to be a highly effective animal camouflage strategy, in which individuals in a population are highly variable in colour and overall coloration pattern. Colour polymorphisms make it difficult for predators to recognise their prey's characteristic colours and patterns (since they are variable). It is challenging for predators to form an accurate search image for prey that vary in their appearance (Dukas and Kamil, 2001, Dukas and Kamil, 2000, Dukas, 2004), which in turn enjoy an increased survivorship as a consequence (Bond and Kamil, 2002, Bond and Kamil, 2006). Just as disruptive coloration functions to disguise edge and shape, polymorphism functions to disguise colour and pattern elements by exploiting the limits of visual attention (for a review see (Bond, 2007)). From findings in vision science, it is well known that colour is an important aspect of object recognition. Therefore, when colour is not consistent, the object becomes less recognisable (Jacobs, 2002) and subsequently more emphasis is placed on shape information for recognition (Tanaka and Presnell, 1999). Together, it would seem that disruptive coloration and colour polymorphism function to impair object recognition (of shape and colour, respectively), whereas background matching functions to reduce detectability. 


\subsection{Future work}

This thesis has tested how camouflage functions, with a particular focus on predictions about how edge-intersecting patches function to disrupt, such as $(i)$ edge coloration increases concealment that is dependent on visibility of shape and boundary properties, (ii) edge coloration breaks up an animal's outline, and (iii) edge coloration impairs object recognition. Similar predictions about how disruption functions can be made for other forms of disruptive markings, such as disruptive stripes, coincidental disruption (Cuthill and Szekely, 2009), face disruption (Harvey, 2013) and distraction markings (Dimitrova et al., 2009, Stevens et al., 2013). Future work could apply the function-centric approach used in my thesis research to studying these addition forms of disruption.

The latter function-centric approach should also be used in evaluating putative disruptive coloration in taxonomically diverse animal species. As noted in the Introduction, surprisingly, "currently, there is no firm evidence for disruptive coloration in mammals" Caro (2009), and this statement is true for all taxa. In this thesis, I demonstrate how to distinguish disruptive coloration from background matching using artificial targets. To assess disruptive coloration in real animal species, I suggest combining this thesis' functional-centric approach with field manipulations of animal coloration, using live animals, stuffed mounts or realistic models (Caro and Melville, 2013), and quantifying coloration treatment effects on prey detection rates. 


\subsection{Conclusions}

This thesis has demonstrated that edge coloration increases prey survivorship, a finding in concordance with several others studies (Cuthill et al., 2006a, Cuthill et al., 2005, Cuthill et al., 2000, Stevens et al., 2006b, Stevens et al., 2009, Schaefer and Stobbe, 2006, Dimitrova et al., 2009, Merilaita and Lind, 2005). It has, however, gone beyond previous work in providing new lines of evidence that this protective aspect of coloration is attributable to a discrete disruptive function. I argue that, to distinguish between categories of camouflage, a functional basis is required to show that different camouflage strategies contribute to concealment independently (Stevens and Merilaita, 2011, Stevens and Merilaita, 2009). This distinction is particularly necessary to disentangle disruptive coloration from background matching, because, from an animal's visual appearance alone, it can be difficult to distinguish between these two types of camouflage strategies. Indeed, Cuthill \& Troskianko (Cuthill and Troscianko, 2011) acknowledge that for disruptive coloration to work in the manner prescribed, object recognition must be impaired, otherwise the theory fails. Edge coloration in artificial moths breaks up their outline, thereby degrading their shape and resulting in impaired object recognition in receivers (human subjects). Collectively, my results suggest that edge coloration is not an artefact of background matching since it continues to improve concealment at the expense of background similarity, and behaves according to functional predictions of disruptive coloration. It is hoped that this function-centric approach and associated methodologies will be utilized in future to better assay other disruptive markings (i.e. bisecting stripes, high-contrast disruption, face disruption \& coincidental disruption) and aid in the identification of disruptive coloration in wild animals - a challenge that still remains at the heart of camouflage research. 


\section{References Cited}

AHNESJO, J. \& FORSMAN, A. 2006. Differential habitat selection by pygmy grasshopper color morphs; interactive effects of temperature and predator avoidance. Evolutionary Ecology, 20, 235-257.

ALLEN, F. H. 1912. Remarks on the case of Roosevelt vs. Thayer, with a few independent suggestions on the concealing coloration question. Auk, 29, 489-507.

ALLEN, W. L., BADDELEY, R., CUTHILL, I. C. \& SCOTT-SAMUEL, N. E. 2012. A Quantitative test of the predicted relationship between countershading and lighting environment. American Naturalist, 180, 762-776.

ALLEN, W. L., CUTHILL, I. C., SCOTT-SAMUEL, N. E. \& BADDELEY, R. 2011. Why the leopard got its spots: relating pattern development to ecology in felids. Proceedings of the Royal Society B-Biological Sciences, 278, 1373-1380.

ANDERSON, B. L. 2013. Filling-in the gaps in models of completion. Cognitive Neuroscience, 4, 48-49.

ANDERSON, B. L., SINGH, M. \& FLEMING, R. W. 2002. The interpolation of object and surface structure. Cognitive Psychology, 44, 148-190.

BAAYEN, R. H. 2011. LanguageR: Data sets and functions with "Analyzing Linguistic Data: A practical introduction to statistics" Cambridge University Press, Cambridge.

BAIN, R. S., RASHED, A., COWPER, V. J., GILBERT, F. S. \& SHERRATT, T. N. 2007. The key mimetic features of hoverflies through avian eyes. Proceedings of the Royal Society B: Biological Sciences, 274, 1949-1954. 
BARBET, I. \& FAGOT, J. 2011. Processing of contour closure by baboons (Papio papio). Journal of Experimental Psychology-Animal Behavior Processes, 37, 407419.

BARBOSA, A., ALLEN, J. J., MATHGER, L. M. \& HANLON, R. T. 2012. Cuttlefish use visual cues to determine arm postures for camouflage. Proceedings of the Royal Society B-Biological Sciences, 279, 84-90.

BARBOSA, A., MATHGER, L. M., BURESCH, K. C., KELLY, J., CHUBB, C., CHIAO, C. C. \& HANLON, R. T. 2008. Cuttlefish camouflage: The effects of substrate contrast and size in evoking uniform, mottle or disruptive body patterns. Vision Research, 48, 1242-1253.

BARBOUR, T. 1913. A different aspect of the case of Roosevelt vs. Thayer. The Auk, 30, $81-91$.

BATES, D., MAECHIER, M. \& BOLKER, B. 2011. Ime4: Linear mixed-effects models using S4 classes, $R$ package version 0.999375-39.

BEATTY, C. D., BAIN, R. S. \& SHERRATT, T. N. 2005. The evolution of aggregation in profitable and unprofitable prey. Animal Behaviour, 70, 199-208.

BECK, J., PRAZDNY, K. \& IVRY, R. 1984. The perception of transparency with achromatic colors. Perception \& Psychophysics, 35, 407-422.

BEHRENS, R. R. 2002. False Colors: art, design and modern camouflage, Dysart, Iowa, Bobolink Books.

BEHRENS, R. R. 2009. Camoupedia, Dysart, Iowa, Bobolink Books.

BELL, J., HANCOCK, S., KINGDOM, F. A. A. \& PEIRCE, J. W. 2010. Global shape processing: Which parts form the whole? Journal of Vision, 10, 13. 
BENNETT, A. T. D. \& CUTHILL, I. C. 1994. Ultraviolet vision in birds: What is its function? Vision Research, 34, 1471-1478.

BENNETT, P. J. \& BANKS, M. S. 1987. Sensitivity loss in odd-symmetrical mechanisms and phase anomalies in peripheral-vision. Nature, 326, 873-876.

BERGEVIN, R. \& LEVINE, M. D. 1992. Extraction of line drawing features for object recognition. Pattern Recognition, 25, 319-334.

BERTAMINI, M. \& LAWSON, R. 2008. Rapid figure-ground responses to stereograms reveal an advantage for a convex foreground. Perception, 37, 483-494.

BETTS, C. R. \& WOOTTON, R. J. 1988. Wing shape and flight behaviour in butterflies (Lepidoptera: Papilionoidea and Hesperioidea): a preliminary analysis. Journal of Experimental Biology, 138, 271-288.

BEX, P. J. \& MAKOUS, W. 2002. Spatial frequency, phase, and the contrast of natural images. J. Opt. Soc. Am. A, 19, 1096-1106.

BINETTI, V. R., SCHIFFMAN, J. D., LEAFFER, O. D., SPANIER, J. E. \& SCHAUER, C. L. 2009. The natural transparency and piezoelectric response of the Greta oto butterfly wing. Integrative Biology, 1, 324-329.

BOND, A. B. 2007. The evolution of color polymorphism: Crypticity searching images, and apostatic selection. Annual Review of Ecology Evolution and Systematics, 38, 489-514.

BOND, A. B. \& KAMIL, A. C. 2002. Visual predators select for crypticity and polymorphism in virtual prey. Nature, 415, 609-613. 
BOND, A. B. \& KAMIL, A. C. 2006. Spatial heterogeneity, predator cognition, and the evolution of color polymorphism in virtual prey. Proceedings of the National Academy of Sciences of the United States of America, 103, 3214-3219.

BOOT, W. R., NEIDER, M. B. \& KRAMER, A. F. 2009. Training and transfer of training in the search for camouflaged targets. Attention Perception \& Psychophysics, 71, 950-963.

BROWN, M. M., KREITER, N. A., MAPLE, J. T. \& SINNOTT, J. M. 1992. Silhouettes elicit alarm calls from captive vervet monkeys (Cercopithecus-aethiops). Journal of Comparative Psychology, 106, 350-359.

BURKE, D., EVERINGHAM, P., ROGERS, T., HINTON, M. \& HALL-ASPLAND, S. 2001. Perceptual grouping in two visually reliant species: Humans (Homo sapiens) and Australian sea lions (Neophoca cinerea). Perception, 30, 1093-1106.

BUSH, S. E., KIM, D., REED, M. \& CLAYTON, D. H. 2010. Evolution of cryptic coloration in ectoparasites. American Naturalist, 176, 529-535.

CALLAHAN, A. 2007. Quantifying crypsis: analyzing the resting site selection of moths in their natural habitats. Intergrated Science B.Sc. thesis, Carleton University.

CARLILE, P. A., PETERS, R. A. \& EVANS, C. S. 2006. Detection of a looming stimulus by the Jacky dragon: selective sensitivity to characteristics of an aerial predator. Animal Behaviour, 72, 553-562.

CARO, T. 2005. The adaptive significance of coloration in mammals. Bioscience, 55, $125-136$.

CARO, T. 2009. Contrasting coloration in terrestrial mammals. Philosophical Transactions of the Royal Society B-Biological Sciences, 364, 537-548. 
CARO, T. 2011. The functions of black-and-white coloration in mammals: review and synthesis, in Animal camouflage: function and mechanism, ed M Stevens \& S Merilaita, Cambridge University Press, pp298-329.

CARO, T. \& MELVILLE, C. 2013. Investigating colouration in large and rare mammals: the case of the giant anteater. Ethology, Ecology \& Evolution, 24, 104-115.

CARO, T. \& STANKOWICH, T. 2010. The function of contrasting pelage markings in artiodactyls. Behavioral Ecology, 21, 78-84.

CARO, T., STANKOWICH, T., MESNICK, S. L., COSTA, D. P. \& BEEMAN, K. 2012. Pelage coloration in pinnipeds: functional considerations. Behavioral Ecology, 23, 765-774.

CAVOTO, B. R. \& COOK, R. G. 2006. The contribution of monocular depth cues to scene perception by pigeons. Psychological Science, 17, 628-634.

CHANG, C. C., LEE, Y. H., LIN, C. J., LIU, B. S. \& SHIH, Y. C. 2012. Visual assessment of camouflaged targets with different background similarities. Perceptual and Motor Skills, 114, 527-541.

CHEN, H., CHAN, C. T. \& SHENG, P. 2010. Transformation optics and metamaterials. Nat. Mater., 9, 387-396.

CHEN HE, X. \& YUNG, N. H. C. 2008. Corner detector based on global and local curvature properties. Optical Engineering, 47.

CHEN, P.-Y. \& ALÙ, A. 2011. Mantle cloaking using thin patterned metasurfaces. Physical Review B, 84, 205110. 
CHIAO, C. C., CHUBB, C., BURESCH, K., SIEMANN, L. \& HANLON, R. T. 2009. The scaling effects of substrate texture on camouflage patterning in cuttlefish. Vision Research, 49, 1647-1656.

CHIAO, C. C., CHUBB, C., BURESCH, K. C., BARBOSA, A., ALLEN, J. J., MATHGER, L. M. \& HANLON, R. T. 2010. Mottle camouflage patterns in cuttlefish: quantitative characterization and visual background stimuli that evoke them. Journal of Experimental Biology, 213, 187-199.

CHIAO, C. C., KELMAN, E. J. \& HANLON, R. T. 2005. Disruptive body patterning of cuttlefish (Sepia officinalis) requires visual information regarding edges and contrast of objects in natural substrate backgrounds. Biological Bulletin, 208, 711.

CHIAO, C. C., ULMER, K. M., SIEMANN, L. A., BURESCH, K. C., CHUBB, C. \& HANLON, R. T. 2013. How visual edge features influence cuttlefish camouflage patterning. Vision Research, 83, 40-47.

CLARKE, J. M. \& SCHLUTER, D. 2011. Colour plasticity and background matching in a threespine stickleback species pair. Biological Journal of the Linnean Society, $102,902-914$.

COOK, L. M., GRANT, B. S., SACCHERI, I. J. \& MALLET, J. 2012. Selective bird predation on the peppered moth: the last experiment of Michael Majerus. Biology Letters, 8, 609-612.

COOPER, J. M. \& ALLEN, J. A. 1994. Selection by wild birds on artificial dimorphic prey on varied backgrounds. Biological Journal of the Linnean Society, 51, 433446. 
COPPOLA, D. M., PURVES, H. R., MCCOY, A. N. \& PURVES, D. 1998. The distribution of oriented contours in the real word. Proceedings of the National Academy of Sciences of the United States of America, 95, 4002-4006.

COTT, H. B. 1940. Adaptive Coloration in Animals, London, Methuen.

COX, S., CHANDLER, S., BARRON, C. \& WORK, K. 2009. Benthic fish exhibit more plastic crypsis than non-benthic species in a freshwater spring. Journal of Ethology, 27, 497-505.

CRAMER, G. 2013. 'Quantum stealth' invisibility cloak. http://www.hyperstealth.com/Quantum-Stealth/index.html [Online]. Hyper Stealth. [Accessed 7/04/13].

CREDIDIO, H. F., TEIXEIRA, E. N., REIS, S. D. S., MOREIRA, A. A. \& ANDRADE, J. S. 2012. Statistical patterns of visual search for hidden objects. Scientific Reports, 2.

CUTHILL, I. C., HIBY, E. \& LLOYD, E. 2006a. The predation costs of symmetrical cryptic coloration. Proceedings of the Royal Society B-Biological Sciences, 273, $1267-1271$

CUTHILL, I. C., PARTRIDGE, J. C., BENNETT, A. T. D., CHURCH, S. C., HART, N. S. \& HUNT, S. 2000. Ultraviolet vision in birds. Adv. Study Behav., 29, 159-214. CUTHILL, I. C., STEVENS, M., SHEPPARD, J., MADDOCKS, T., PARRAGA, C. A. \& TROSCIANKO, T. S. 2005. Disruptive coloration and background pattern matching. Nature, 434, 72-74. 
CUTHILL, I. C., STEVENS, M., WINDSOR, A. M. M. \& WALKER, H. J. 2006b. The effects of pattern symmetry on detection of disruptive and background-matching coloration. Behavioral Ecology, 17, 828-832.

CUTHILL, I. C. \& SZEKELY, A. 2009. Coincident disruptive coloration. Philosophical Transactions of the Royal Society B-Biological Sciences, 364, 489-496.

CUTHILL, I. C. \& TROSCIANKO, T. S. 2011. Animal camouflage: biology meets psychology, computer science and art. In: BREBBIA, C. A., GREATED, C. \& COLLINS, M. W. (eds.) Colour in art, design and nature. Southamton, Boston: WIT Press.

DARWIN, C. R. 1859. On the origin of species by the means of natural selection. London: John Murray.

DAWKINS, M. 1971. Perceptual changes in chicks: Another look at the 'search image' concept. Animal Behaviour, 19, 566-574.

DAYTON, G. H., SAENZ, D., BAUM, K. A., LANGERHANS, R. B. \& DEWITT, T. J. 2005. Body shape, burst speed and escape behavior of larval anurans. Oikos, 111, $582-591$.

DELVENNE, J. F. \& DENT, K. 2008. Distinctive shapes benefit short-term memory for color associations, but not for color. Perception \& Psychophysics, 70, 1024-1031.

DERUELLE, C., BARBET, I., DEPY, D. \& FAGOT, J. 2000. Perception of partly occluded figures by baboons (Papio papio). Perception, 29, 1483-1497.

DI LOLLO, V., ENNS, J. T. \& RENSINK, R. A. 2000. Competition for consciousness among visual events: The psychophysics of reentrant visual processes. Journal of Experimental Psychology-General, 129, 481-507. 
DIMITROVA, M. \& MERILAITA, S. 2009. Prey concealment: visual background complexity and prey contrast distribution. Behavioral Ecology, 21, 176-181.

DIMITROVA, M. \& MERILAITA, S. 2010. Prey concealment: visual background complexity and prey contrast distribution. Behavioral Ecology, 21, 176-181.

DIMITROVA, M. \& MERILAITA, S. 2012. Prey pattern regularity and background complexity affect detectability of background-matching prey. Behavioral Ecology, 23, 384-390.

DIMITROVA, M., STOBBE, N., SCHAEFER, H. M. \& MERILAITA, S. 2009. Concealed by conspicuousness: distractive prey markings and backgrounds. Proceedings of the Royal Society B-Biological Sciences, 276, 1905-1910.

DUCHOWSKI, A. T. 2002. A breadth-first survey of eye-tracking applications. Behavior Research Methods Instruments \& Computers, 34, 455-470.

DUKAS, R. 2004. Causes and consequences of limited attention. Brain Behavior and Evolution, 63, 197-210.

DUKAS, R. \& KAMIL, A. C. 2000. The cost of limited attention in blue jays. Behavioral Ecology, 11, 502-506.

DUKAS, R. \& KAMIL, A. C. 2001. Limited attention: the constraint underlying search image. Behavioral Ecology, 12, 192-199.

EDMUNDS, M. \& DEWHIRST, R. A. 1994. The survival value of countershading with wild birds as predators. Biological Journal of the Linnean Society, 51, 447-452.

EINHAUSER, W., MOELLER, G. U., SCHUMANN, F., CONRADT, J., VOCKEROTH, J., BARD, K., SCHNEIDER, E. \& KONIG, P. 2009. Eye-head coordination during free exploration in human and cat. In: STRUPP, M., 
BUTTNER, U. \& COHEN, B. (eds.) Basic and Clinical Aspects of Vertigo and Dizziness. Oxford: Blackwell Publishing.

ELDER, J. H., KRUPNIK, A. \& JOHNSTON, L. A. 2003. Contour grouping with prior models. IEEE Transactions on Pattern Analysis and Machine Intelligence, 25, 661-674.

ELDER, J. H. \& VELISAVLJEVIC, L. 2009. Cue dynamics underlying rapid detection of animals in natural scenes. Journal of Vision, 9, 1-20.

ENDLER, J. A. 1980. Natural-selection on color patterns in Poecilia-reticulata. Evolution, 34, 76-91.

ENDLER, J. A. 1982. Convergent and divergent effects of natural-selection on color patterns in 2 fish faunas. Evolution, 36, 178-188.

ENDLER, J. A. 1984. Progressive background in moths, and a quantitative measure of crypsis. Biological Journal of the Linnean Society, 22, 187-231.

ENDLER, J. A. 2006. Commentary - Disruptive and cryptic coloration. Proceedings of the Royal Society B-Biological Sciences, 273, 2425-2426.

ENDLER, J. A. \& BASOLO, A. L. 1998. Sensory ecology, receiver biases and sexual selection. Trends in Ecology \& Evolution, 13, 415-420.

ERIKSEN, C. W. \& YEH, Y. Y. 1985. Allocation of attention in the visual-field. Journal of Experimental Psychology-Human Perception and Performance, 11, 583-597.

EVANS, K. M., JACOBS, R. A., TARDUNO, J. A. \& PELZ, J. B. 2012. Collecting and analyzing eye tracking data in outdoor environments. Journal Eye Movement Research, 5, 1-9. 
FAGOT, J. \& TOMONAGA, M. 2001. Effects of element separation on perceptual grouping by humans (Homo sapiens) and chimpanzees (Pan troglodytes): perception of Kanizsa illusory figures. Animal Cognition, 4, 171-177.

FECTEAU, J. H. \& MUNOZ, D. P. 2006. Salience, relevance, and firing: a priority map for target selection. Trends in Cognitive Sciences, 10, 382-390.

FELTMATE, B. W. \& WILLIAMS, D. D. 1989. A test of crypsis and predator avoidance in the stonefly Paragnetina media (Plecoptera: Perlidae). Animal Behaviour, 37, 992-999.

FERNÁNDEZ-JURICIC, E., BEAUCHAMP, G., TREMINIO, R. \& HOOVER, M. 2011. Making heads turn: association between head movements during vigilance and perceived predation risk in brown-headed cowbird flocks. Animal Behaviour, $82,573-577$.

FIELD, D. J., HAYES, A. \& HESS, R. F. 1993. Contour integration by the human visual system: Evidence for a local "association field". Vision Research, 33, 173-193.

FORSMAN, A., KARLSSON, M., WENNERSTEN, L., JOHANSSON, J. \& KARPESTAM, E. 2011. Rapid evolution of fire melanism in replicated populations of pygmy grasshoppers. Evolution, 65, 2530-2540.

FRASER, S., CALLAHAN, A., KLASSEN, D. \& SHERRATT, T. N. 2007. Empirical tests of the role of disruptive coloration in reducing detectability. Proceedings of the Royal Society B-Biological Sciences, 274, 1325-1331.

FRAZOR, R. A. \& GEISLER, W. S. 2006. Local luminance and contrast in natural images. Vision Research, 46, 1585-1598. 
FUJITA, K. 2001. Perceptual completion in rhesus monkeys (Macaca mulatta) and pigeons (Columba livia). Perception \& Psychophysics, 63, 115-125.

GARCIA, T. S. \& SIH, A. 2003. Color change and color-dependent behavior in response to predation risk in the salamander sister species Ambystoma barbouri and Ambystoma texanum. Oecologia, 137, 131-139.

GEISLER, W. S., PERRY, J. S., SUPER, B. J. \& GALLOGLY, D. P. 2001. Edge cooccurrence in natural images predicts contour grouping performance. Vision Research, 41, 711-724.

GIGUÈRE, L. A. \& NORTHCOTE, T. G. 1987. Ingested prey increase risks of visual predation in transparent Chaoborus larvae. Oecologia, 73, 48-52.

GIRARD, P. \& KOENIG-ROBERT, R. 2011. Ultra-rapid categorization of fourierspectrum equalized natural images: macaques and humans perform similarly. PLoS ONE, 6, 1-13.

GOULD, S. J. 1991. Red wings in the sunset in Bullt for Brontosaurus, New York, W. W. Norton, pp 209-228

GRAYSON, J., EDMUNDS, M., EVANS, E. H. \& BRITTON, G. 1991. Carotenoids and coloration of poplar hawkmoth caterpillars (Laothoe populi). Biological Journal of the Linnean Society, 42, 457-465.

HACKER, S. D. \& MADIN, L. P. 1991. Why habitat architecture and color are important to shrimps living in pelagic sargassum - use of camouflage and plant-part mimicry. Marine Ecology Progress Series, 70, 143-155.

HALKO, M. A., MINGOLLA, E. \& SOMERS, D. C. 2008. Multiple mechanisms of illusory contour perception. Journal of Vision, 8, 1-17. 
HART, N. S. \& HUNT, D. M. 2007. Avian visual pigments: characteristics, spectral tuning, and evolution. Am. Nat., 169, S7-S26.

HARVEY, A. 2013. Computer vision dazzle for face detection camouflage [Online]. Available: www.cvdazzle.com.

HAYHOE, M. \& BALLARD, D. 2005. Eye movements in natural behavior. Trends in Cognitive Sciences, 9, 188-194.

HEITGER, F., VON DER HEYDT, R., PETERHANS, E., ROSENTHALER, L. \& KUBLER, O. 1998. Simulation of neural contour mechanisms: representing anomalous contours. Image and Vision Computing, 16, 407-421.

HELSON, H. 1933. The fundamental propositions of Gestalt psychology. Psychological Review, 40, 13-32.

HESS, R. F. \& DAKIN, S. C. 1997. Absence of contour linking in peripheral vision. Nature, 390, 602-604.

HESS, R. F. \& DAKIN, S. C. 1999. Contour integration in the peripheral field. Vision Research, 39, 947-959.

HODOS, W. 1993. The visual capabilities of birds. In: ZEIGLER, H. P. \& BISCHOF, H. J. (eds.) Vision, Brain and Behaviour in Birds. Boston, MIT Press.

HOFFMAN, J. E. \& NELSON, B. 1981. Spatial selectivity in visual-search. Perception \& Psychophysics, 30, 283-290.

HORRIDGE, G. A., ZHANG, S. W. \& OCARROLL, D. 1992. Insect perception of illusory contours. Philosophical Transactions of the Royal Society of London Series B-Biological Sciences, 337, 59-64. 
HOUSTON, A. I., STEVENS, M. \& CUTHILL, I. C. 2007. Animal camouflage:

compromise or specialize in a 2 patch-type environment? Behavioral Ecology, 18, $769-775$.

HUBEL, D. H. \& WIESEL, T. N. 1968. Receptive fields and functional architecture of monkey striate cortex. Journal of Physiology-London, 195, 215-243.

HULTGREN, K. M. \& STACHOWICZ, J. J. 2009. Evolution of decoration in majoid crabs: a comparative phylogenetic analysis of the role of body size and alternative defensive strategies. American Naturalist, 173, 566-578.

HULTGREN, K. M. \& STACHOWICZ, J. J. 2010. Size-related habitat shifts facilitated by positive preference induction in a marine kelp crab. Behavioral Ecology, 21, 329-336.

INGS, T. C., WANG, M. Y. \& CHITTKA, L. 2012. Colour-independent shape recognition of cryptic predators by bumblebees. Behavioral Ecology and Sociobiology, 66, 487-496.

ITTI, L. \& KOCH, C. 2001. Computational modelling of visual attention. Nature Reviews Neuroscience, 2, 194-203.

JACOBS, R. A. 2002. What determines visual cue reliability? Trends in Cognitive Sciences, 6, 345-350.

JOHANNESSON, K. \& EKENDAHL, A. 2002. Selective predation favouring cryptic individuals of marine snails (Littorina). Biological Journal of the Linnean Society, 76, 137-144.

JOHNSEN, S. 2001. Hidden in Plain Sight: The Ecology and Physiology of Organismal Transparency. The Biological Bulletin, 201, 301-318. 
JOHNSON, S. P., AMSO, D. \& SLEMMER, J. A. 2003. Development of object concepts in infancy: Evidence for early learning in an eye-tracking paradigm. Proceedings of the National Academy of Sciences of the United States of America, 100, 1056810573.

JONES, M. J., SINHA, P., VETTER, T. \& POGGIO, T. 1997. Top down learning of lowlevel vision tasks. Current biology, 7, 991-994.

JULESZ, B. (ed.) 1984. Dynamic aspects of neocortical function.

KANG, C. K., MOON, J. Y., LEE, S. I. \& JABLONSKI, P. G. 2012. Camouflage through an active choice of a resting spot and body orientation in moths. Journal of Evolutionary Biology, 25, 1695-1702.

KANIZSA, G. \& GERBINO, W. 1976. Convexity and symmetry in figure-ground organization. Vision and artefact, Springer, New York, 25-32.

KANIZSA, G., RENZI, P., CONTE, S., COMPOSTELA, C. \& GUERANI, L. 1993. Amodal completion in mouse vision. Perception, 22, 713-721.

KARPESTAM, E., MERILAITA, S. \& FORSMAN, A. 2012. Reduced predation risk for melanistic pygmy grasshoppers in post-fire environments. Ecology and Evolution, 2, 2204-2212.

KATS, L. B. \& VANDRAGT, R. G. 1986. Background color-matching in the spring peeper, hyla-crucifer. Copeia, 109-115.

KELLMAN, P. J. \& SHIPLEY, T. F. 1991. A theory of visual interpolation in object perception. Cognitive Psychology, 23, 141-221.

KELMAN, E. J., BADDELEY, R. J., SHOHET, A. J. \& OSORIO, D. 2007. Perception of visual texture and the expression of disruptive camouflage by the cuttlefish, 
Sepia officinalis. Proceedings of the Royal Society B-Biological Sciences, 274, 1369-1375.

KENNEDY, J. M. \& DOMANDER, R. 1985. Shape and contour - the points of maximum change are least useful for recognition. Perception, 14, 367-370.

KETTLEWELL, H. B. D. 1956. A resume of investigations on the evolution of melanism in the Lepidoptera. Proceedings of the Royal Society B-Biological Sciences, 145, 297-303.

KETTLEWELL, H. B. D. \& CONN, D. L. T. 1977. Further Background-Choice Experiments on Cryptic Lepidoptera. Journal of Zoology, 181, 371-376.

KILTIE, R. A. 1988. Countershading: Universally deceptive or deceptively universal? Trends in Ecology \& Evolution, 3, 21-23.

KINGSLAND, S. 1978. Abbott Thayer and the protective coloration debate. Journal of the History of Biology, 11, 223-244.

KJAERSGAARD, A., PERTOLDI, C., LOESCHCKE, V. \& HANSEN, D. W. 2008. Tracking the gaze of birds. Journal of Avian Biology, 39, 466-469.

KJERNSMO, K. \& MERILAITA, S. 2012. Background choice as an anti-predator strategy: the roles of background matching and visual complexity in the habitat choice of the least killifish. Proceedings of the Royal Society B-Biological Sciences, 279, 4192-4198.

KLEIN, R. M. 2000. Inhibition of return. Trends in Cognitive Sciences, 4, 138-147.

KLEIN, R. M. \& MACINNES, W. J. 1999. Inhibition of return is a foraging facilitator in visual search. Psychological Science, 10, 346-352. 
KNILL, R. \& ALLEN, J. A. 1995. Does polymorphism protect? An experiment with human 'predators'. Ethology, 99, 127-138.

KOURTZI, Z., BETTS, L. R., SARKHEIL, P. \& WELCHMAN, A. E. 2005. Distributed Neural Plasticity for Shape Learning in the Human Visual Cortex. PLoS Biology, 3, 1317-1327.

KUBODERA, T., KOYAMA, Y. \& MORI, K. 2007. Observations of wild hunting behaviour and bioluminescence of a large deep-sea, eight-armed squid, Taningia danae. Proceedings of the Royal Society B: Biological Sciences, 274, 1029-1034.

KURYLO, D. D. 2008. Effects of visual cortex lesions on perceptual grouping in rats. Behavioural Brain Research, 190, 182-188.

LABERGE, D. 1983. Spatial extent of attention to letters and words. Journal of Experimental Psychology-Human Perception and Performance, 9, 371-379.

LANGERHANS, R. B. 2009. Morphology, performance, fitness: functional insight into a post-Pleistocene radiation of mosquitofish. Biology Letters, 5, 488-491.

LEE, T. S. \& MUMFORD, D. 2003. Hierarchical Bayesian inference in the visual cortex. Journal of the Optical Society of America a-Optics Image Science and Vision, 20, $1434-1448$

LEHRER, M. \& CAMPAN, R. 2004. Shape discrimination by wasps (Paravespula germanica) at the food source: generalization among various types of contrast. Journal of Comparative Physiology a-Neuroethology Sensory Neural and Behavioral Physiology, 190, 651-663.

LEVY, J., FOULSHAM, T. \& KINGSTONE, A. 2013. Monsters are people too. Biology Letters, 9, 23-31. 
LIEBE, S., FISCHER, E., LOGOTHETIS, N. K. \& RAINER, G. 2009. Color and shape interactions in the recognition of natural scenes by human and monkey observers. Journal of Vision, 9, 1-16.

LIMA, S. L. \& DILL, L. M. 1990. Behavioral decisions made under the risk of predation: a review and prospectus. Canadian Journal of Zoology, 68, 619-640.

LIVERSEDGE, S. P. \& FINDLAY, J. M. 2000. Saccadic eye movements and cognition. Trends in Cognitive Sciences, 4, 6-14.

LLOYD-JONES, T. \& LUCKHURST, L. 2002. Outline shape is a mediator of object recognition that is particularly important for living things. Memory \& Cognition, $30,489-498$.

LLOYD-JONES, T. J., GEHRKE, J. \& LAUDER, J. 2010. Animal recognition and eye movements the contribution of outline contour and local feature information. Experimental Psychology, 57, 117-125.

LOGOTHETIS, N. K., PAULS, J. \& POGGIO, T. 1995. Shape representation in the inferior temporal cortex of monkeys. Curr. Biol., 5, 552-563.

LUDWIG, C. J. H. \& GILCHRIST, I. D. 2006. The relative contributions of luminance contrast and task demands on saccade target selection. Vision Research, 46, 27432748.

LUNDVALL, D., SVANBACK, R., PERSSON, L. \& BYSTROM, P. 1999. Sizedependent predation in piscivores: interactions between predator foraging and prey avoidance abilities. Canadian Journal of Fisheries and Aquatic Sciences, 56, 1285-1292. 
MACÉ, M. M., DELORME, A., RICHARD, G. \& FABRE-THORPE, M. 2010. Spotting animals in natural scenes: efficiency of humans and monkeys at very low contrasts. Animal Cognition, 13, 405-418.

MAROTTI, R. B., PAVAN, A. \& CASCO, C. 2012. The integration of straight contours (snakes and ladders): The role of spatial arrangement, spatial frequency and spatial phase. Vision Research, 71, 44-52.

MARR, D. \& HILDRETH, E. 1980. Theory of edge detection. Proceedings of the Royal Society of London B, 207, 187-217.

MATHGER, L. M., CHIAO, C. C., BARBOSA, A., BURESCH, K. C., KAYE, S. \& HANLON, R. T. 2007. Disruptive coloration elicited on controlled natural substrates in cuttlefish, Sepia officinalis. Journal of Experimental Biology, 210, 2657-2666.

MATIN, E. 1974. Saccadic suppression - review and an analysis. Psychological Bulletin, 81, 899-917.

MCFALL-NGAI, M. J. 1990. Crypsis in the Pelagic Environment. American Zoologist, $30,175-188$.

MERILAITA, S. 1998. Crypsis through disruptive coloration in an isopod. Proceedings of the Royal Society of London Series B-Biological Sciences, 265, 1059-1064.

MERILAITA, S. 2003. Visual background complexity facilitates the evolution of camouflage. Evolution, 57, 1248-1254.

MERILAITA, S. \& LIND, J. 2005. Background-matching and disruptive coloration, and the evolution of cryptic coloration. Proceedings of the Royal Society B-Biological Sciences, 272, 665-670. 
MERILAITA, S., TUOMI, J. \& JORMALAINEN, V. 1999. Optimization of cryptic coloration in heterogeneous habitats. Biological Journal of the Linnean Society, $67,151-161$.

MEYER-ROCHOW, V. B. 1974. Leptocephali and other transparentfish larvaefromthe south-eastern Atlantic ocean. Zool. Anz, 192, 240-251.

MOSS, R., JACKSON, R. R. \& POLLARD, S. D. 2006. Hiding in the grass: background matching conceals moths (Lepidoptera : Crambidae) from detection by spider eyes (Araneae : Salticidae). New Zealand Journal of Zoology, 33, 207-214.

NAGASAKA, Y., HORI, K. \& OSADA, Y. 2005. Perceptual grouping in pigeons. Perception, 34, 625-632.

NAKAMURA, N., WATANABE, S., BETSUYAKU, T. \& FUJITA, K. 2010. Do Bantams (Gallus gallus domesticus) Experience Amodal Completion? An Analysis of Visual Search Performance. Journal of Comparative Psychology, 124, $331-335$

NIEDER, A. \& WAGNER, H. 1999. Perception and neuronal coding of subjective contours in the owl. Nature Neuroscience, 2, 660-663.

NIELSEN, K. J. \& RAINER, G. 2007. Object recognition: similar visual strategies of birds and mammals. Current biology : $C B, 17, \mathrm{R} 174-\mathrm{R} 176$.

NUERNBERG, S., ABERHAN, M. \& KRAUSE, R. A. 2012. Evolutionary and ecological patterns in body size, shape, and ornamentation in the Jurassic bivalve Chlamys (Chlamys) textoria (Schlotheim, 1820). Fossil Record, 15, 27-39.

NYBERG, S. \& BOHMAN, L. 2001. Assessing camouflage using textural features. Proceedings of SPIE, 3470, 60-71. 
O'CONNOR, D. H., FUKUI, M. M., PINSK, M. A. \& KASTNER, S. 2002. Attention modulates responses in the human lateral geniculate nucleus. Nature Neuroscience, 5, 1203-1209.

OHAYON, S., HARMENING, W., WAGNER, H. \& RIVLIN, E. 2008. Through a barn owl's eyes: interactions between scene content and visual attention. Biological Cybernetics, 98, 115-132.

OSORIO, D. \& SRINIVASAN, M. V. 1991. Camouflage by Edge Enhancement in Animal Coloration Patterns and Its Implications for Visual Mechanisms. Proceedings of the Royal Society of London. Series B: Biological Sciences, 244, $81-85$.

PALMER, J., VERGHESE, P. \& PAVEL, M. 2000. The psychophysics of visual search. Vision Research, 40, 1227-1268.

PALMER, S. E. 1999. Vision Science: Photons to Phenomenology, Boston, MIT Press.

PALMERI, T. J. \& GAUTHIER, I. 2004. Visual object understanding. Nat Rev Neurosci., 5, 291-303.

PANIS, S., DE WINTER, J., VANDEKERCKHOVE, J. \& WAGEMANS, J. 2008. Identification of everyday objects on the basis of fragmented outline versions. Perception, 37, 271-289.

PANIS, S. \& WAGEMANS, J. 2009. Time-course contingencies in perceptual organization and identification of fragmented object outlines. Journal of Experimental Psychology-Human Perception and Performance, 35, 661-687.

PARADIS, E. 2006. Analysis of Phylogenetics and Evolution with R. 
PARRON, C. \& FAGOT, J. 2007. Comparison of grouping abilities in humans (Homo sapiens) and baboons (Papio papio) with the Ebbinghaus illusion. Journal of Comparative Psychology, 121, 405-411.

PEISSIG, J. J., YOUNG, M. E., WASSERMAN, E. A. \& BIEDERMAN, I. 2005. The role of edges in object recognition by pigeons. Perception, 34, 1353-1374.

PELLISSIER, L., WASSEF, J., BILAT, J., BRAZZOLA, G., BURI, P., COLLIARD, C., FOURNIER, B., HAUSSER, J., YANNIC, G. \& PERRIN, N. 2011. Adaptive colour polymorphism of Acrida ungarica H. (Orthoptera: Acrididae) in a spatially heterogeneous environment. Acta Oecologica-International Journal of Ecology, 37, 93-98.

PENNEY, H. D., HASSALL, C., SKEVINGTON, J. H., ABBOTT, K. R. \& SHERRATT, T. N. 2012. A comparative analysis of the evolution of imperfect mimicry. Nature, 483, 461-466.

PIETREWICZ, A. T. \& KAMIL, A. C. 1977. Visual detection of cryptic prey by Blue Jays (Cyanocitta cristata). Science, 195, 580-582.

POSNER, M. I. 1980. Orienting of attention. Quarterly Journal of Experimental Psychology, 32, 2-25.

POULTON, E. B. 1888. Notes in 1887 upon Lepidopterus Larvae. Transactions of the Entomological Society of London, pp. 595-596.

POULTON, E. B. 1890. The Colours of Animals, London, Kegan Paul, Trench, Trübner \& Co Ltd. 
RAO, R. P. N. \& BALLARD, D. H. 1999. Predictive coding in the visual cortex: a functional interpretation of some extra-classical receptive-field effects. Nature Neuroscience, 2, 79-87.

RAY, S. 2002. Applied photographic optics: lenses and optical systems for photography. Cambridge, MA: Focal Press.

REGOLIN, L. \& VALLORTIGARA, G. 1995. Perception of partly occluded objects by young chicks. Perception \& Psychophysics, 57, 971-976.

RENSINK, R. A., OREGAN, J. K. \& CLARK, J. J. 1997. To see or not to see: The need for attention to perceive changes in scenes. Psychological Science, 8, 368-373.

RENTSCHLER, I. \& TREUTWEIN, B. 1985. Loss of Spatial Phase-Relationships in Extrafoveal Vision. Nature, 313, 308-310.

ROWLAND, H. M., SPEED, M. P., RUXTON, G. D., EDMUNDS, M., STEVENS, M. \& HARVEY, I. F. 2007. Countershading enhances cryptic protection: an experiment with wild birds and artificial prey. Animal Behaviour, 74, 1249-1258.

RUBIN, E. 1915. Visuell wahrgenommene figuren. Glydendalske, Copenhagen.

RUST, N. C. \& DICARLO, J. J. 2010. Selectivity and Tolerance ("Invariance") Both Increase as Visual Information Propagates from Cortical Area V4 to IT. Journal of Neuroscience, 30, 12978-12995.

RUXTON, G. D. 2002. The possible fitness benefits of striped coat coloration for zebra. Mammal Review, 32, 237-244.

RUXTON, G. D. 2009. Non-visual crypsis: a review of the empirical evidence for camouflage to senses other than vision. Philosophical Transactions of the Royal Society B: Biological Sciences, 364, 549-557. 
RUXTON, G. D., SHERRATT, T. N. \& SPEED, M. 2004a. Avoiding Attack: The Evolutionary Ecology of Crypsis, Warning Signals and Mimicry, Oxford University Press.

RUXTON, G. D., SHERRATT, T. N. \& SPEED, M. P. 2004b. Avoiding attack: the evolutionary ecology of crypsis, warning signals and mimicry, Oxford University Press.

SANDOVAL, C. P. 1994. Differential visual predation on morphs of Timema cristinae (Phasmatodeae:Timemidae) and its consequences for host range. Biological Journal of the Linnean Society, 52, 341-356.

SANTANA, S. E., DIAL, T. O., EITING, T. P. \& ALFARO, M. E. 2011. Roosting Ecology and the Evolution of Pelage Markings in Bats. PLoS ONE, 6.

SARGENT, T. D. 1966. Background selection of geometrid and Noctuid moths. Science, $154,1674-1675$.

SARGENT, T. D. 1968. Cryptic moths - effects on background selections of painting circumocular scales. Science, 159, 100-101.

SARGENT, T. D. 1969a. Background selections of pale and melanic forms of cryptic moth Phigalia titea (Cramer). Nature, 222, 585-586.

SARGENT, T. D. 1969b. Behavioral adaptations of cryptic moths .2. Experimental studies on bark-like species. Journal of the New York Entomological Society, 77, $75-79$.

SARGENT, T. D. 1969c. Behavioral adaptations of cryptic moths .V. Preliminary studies on an anthophilous species, schinia Florida (Noctuidae). Journal of the New York Entomological Society, 77, 123-\&. 
SARGENT, T. D. 1969d. Behavioural adaptations of cryptic moths .3. Resting attitudes of two bark-like species, Melanolophia canadaria and Catocala ultronia. Animal Behaviour, 17, 670-672.

SARGENT, T. D. \& KEIPER, R. R. 1969. Behavioural adaptation of cryptic moths. I. Preliminary studies on bark-like species. Journal of the Lepidopterists' Society, $23,1-9$.

SATO, A., KANAZAWA, S. \& FUJITA, K. 1997. Perception of object unity in a chimpanzee (Pan troglodytes). Japanese Psychological Research, 39, 191-199.

SCHAEFER, H. M. \& STOBBE, N. 2006. Disruptive coloration provides camouflage independent of background matching. Proceedings of the Royal Society BBiological Sciences, 273, 2427-2432.

SCHLUESSEL, V., FRICKE, G. \& BLECKMANN, H. 2012. Visual discrimination and object categorization in the cichlid Pseudotropheus sp. Animal Cognition, 15, 525537.

SCOTT, P. M. 1961. The eye of the wind. Hodder \& Stoughton, London.

SHERRATT, T. N. \& BEATTY, C. D. 2003. The evolution of warning signals as reliable indicators of prey defense. The American Naturalist, 162, 377-389.

SHERRATT, T. N., RASHED, A. \& BEATTY, C. D. 2005. Hiding in plain sight. Trends in Ecology \& Evolution, 20, 414-416.

SILBERGLIED, R. E., ANIELLO, A. \& WINDSOR, D. M. 1980. Disruptive coloration in butterflies: lack of support in Anartia fatima. Science, 209, 617-619.

SINGH, M. 2004. Modal and amodal completion generate different shapes. Psychological Science, 15, 454-459. 
SINGH, M. \& ANDERSON, B. L. 2002a. Perceptual assignment of opacity to translucent surfaces: The role of image blur. Perception, 31, 531-552.

SINGH, M. \& ANDERSON, B. L. 2002b. Toward a perceptual theory of transparency. Psychological Review, 109, 492-519.

SINGH, M. \& FULVIO, J. M. 2005. Visual extrapolation of contour geometry. Proceedings of the National Academy of Sciences of the United States of America, 102, 939-944.

SKELHORN, J., ROWLAND, H. M., DELF, J., SPEED, M. P. \& RUXTON, G. D. 2011. Density-dependent predation influences the evolution and behavior of masquerading prey. Proceedings of the National Academy of Sciences of the United States of America, 108, 6532-6536.

SKELHORN, J., ROWLAND, H. M., SPEED, M. P. \& RUXTON, G. D. 2010. Masquerade: Camouflage Without Crypsis. Science, 327, 51-51.

SKELHORN, J. \& RUXTON, G. D. 2010. Predators are less likely to misclassify masquerading prey when their models are present. Biology Letters, 6, 597-599.

SKELHORN, J. \& RUXTON, G. D. 2013. Size-dependent microhabitat selection by masquerading prey. Behavioral Ecology, 24, 89-97.

SMITH, H. M. 2003. Shape variation in Australian Poltys species (Araneae: Araneidae). Bulletin of the British Arachnological Society, 12, 355-360.

SOTO, F. A. \& WASSERMAN, E. A. 2012. Visual object categorization in birds and primates: Integrating behavioral, neurobiological, and computational evidence within a "general process" framework. Cognitive Affective \& Behavioral Neuroscience, 12, 220-240. 
SOVRANO, V. A. \& BISAZZA, A. 2008. Recognition of partly occluded objects by fish. Animal Cognition, 11, 161-166.

SPINOZZI, G., DE LILLO, C., TRUPPA, V. \& CASTORINA, G. 2009. The relative use of proximity, shape similarity, and orientation as visual perceptual grouping cues in tufted capuchin monkeys (Cebus apella) and humans (Homo sapiens). Journal of Comparative Psychology, 123, 56-68.

SRYGLEY, R. B. 2007. Evolution of the wave: aerodynamic and aposematic functions of butterfly wing motion. Proceedings of the Royal Society B-Biological Sciences, 274, 913-917.

STEVENS, M. 2007. Predator perception and the interrelation between different forms of protective coloration. Proceedings of the Royal Society B-Biological Sciences, $274,1457-1464$.

STEVENS, M. \& CUTHILL, I. C. 2006. Disruptive coloration, crypsis and edge detection in early visual processing. Proceedings of the Royal Society BBiological Sciences, 273, 2141-2147.

STEVENS, M., CUTHILL, I. C., PARRAGA, C. A. \& TROSCIANKO, T. 2006a. The effectiveness of disruptive coloration as a concealment strategy. Progress in Brain Research, 155, 49-64.

STEVENS, M., CUTHILL, I. C., WINDSOR, A. M. M. \& WALKER, H. J. 2006 b. Disruptive contrast in animal camouflage. Proceedings of the Royal Society BBiological Sciences, 273, 2433-2438. 
STEVENS, M., MARSHALL, K. L. A., TROSCIANKO, J., FINLAY, S., BURNAND, D. \& CHADWICK, S. L. 2013. Revealed by conspicuousness: distractive markings reduce camouflage. Behavioral Ecology, 24, 213-222.

STEVENS, M. \& MERILAITA, S. 2009. Defining disruptive coloration and distinguishing its functions. Philosophical Transactions of the Royal Society BBiological Sciences, 364, 481-488.

STEVENS, M. \& MERILAITA, S. 2011. Animal camouflage: function and mechanisms, Cambridge University Press.

STEVENS, M., WINNEY, I. S., CANTOR, A. \& GRAHAM, J. 2009. Outline and surface disruption in animal camouflage. Proceedings of the Royal Society BBiological Sciences, 276, 781-786.

STOBBE, N. \& SCHAEFER, H. M. 2008. Enhancement of chromatic contrast increases predation risk for striped butterflies. Proceedings of the Royal Society BBiological Sciences, 275, 1535-1541.

STONER, C. J., BININDA-EMONDS, O. R. P. \& CARO, T. I. M. 2003. The adaptive significance of coloration in lagomorphs. Biological Journal of the Linnean Society, 79, 309-328.

STUART-FOX, D. \& MOUSSALLI, A. 2009. Camouflage, communication and thermoregulation: lessons from colour changing organisms. Philosophical Transactions of the Royal Society B-Biological Sciences, 364, 463-470.

TANAKA, J. W. \& PRESNELL, L. M. 1999. Color diagnosticity in object recognition. Perception \& Psychophysics, 61, 1140-1153. 
TANKUS, A. \& YESHURUN, Y. 2001. Convexity-Based Visual Camouflage Breaking. Computer Vision and Image Understanding, 82, 208-237.

THAYER, A. H. 1896. The law which underlies protective coloration. Auk, 13, 124-129.

THAYER, A. H. 1909. Concealing coloration in the animal kingdom: an exposition of the laws of disguise through color and pattern, London, Macmillan.

THORPE, S. J., GEGENFURTNER, K. R., FABRE-THORPE, M. \& BULTHOFF, H. H. 2001. Detection of animals in natural images using far peripheral vision. European Journal of Neuroscience, 14, 869-876.

TINBERGEN, N. 1963. On aims and methods of Ethology. Zeitschrift für Tierpsychologie, 20, 410-433.

TREISMAN, A. 1988. Features and objects - The 14th Bartlett Memorial Lecture. Quarterly Journal of Experimental Psychology Section a-Human Experimental Psychology, 40, 201-237.

TREISMAN, A. M. \& GELADE, G. 1980. Feature-integration theory of attention. Cognitive Psychology, 12, 97-136.

TROSCIANKO, T., BENTON, C. P., LOVELL, P. G., TOLHURST, D. J. \& PIZLO, Z. 2009. Camouflage and visual perception, Cambridge University Press.

TRUPPA, V., SOVRANO, V. A., SPINOZZI, G. \& BISAZZA, A. 2010. Processing of visual hierarchical stimuli by fish (Xenotoca eiseni). Behavioural Brain Research, 207, 51-60.

TSOTSOS, J. K. 1990. Analyzing vision at the complexity level. Behavioral and Brain Sciences, 13, 423-444. 
TSURUI, K., HONMA, A. \& NISHIDA, T. 2010. Camouflage Effects of Various Colour-Marking Morphs against Different Microhabitat Backgrounds in a Polymorphic Pygmy Grasshopper Tetrix japonica. PLoS ONE, 5, 1-7.

TURING, A. M. 1952. The chemical basis of morphogenesis. Philos. Trans. R. Soc. London Ser. B 237.

TVARDIKOVA, K. \& FUCHS, R. 2010. Tits use amodal completion in predator recognition: a field experiment. Animal Cognition, 13, 609-615.

VALENTINE, J., LI, J., ZENTGRAF, T., BARTAL, G. \& ZHANG, X. 2009. An optical cloak made of dielectrics. Nat. Mater., 8, 568-571.

VAN BUSKIRK, J. \& MCCOLLUM, S. A. 2000. Functional mechanisms of an inducible defence in tadpoles: morphology and behaviour influence mortality risk from predation. Journal of Evolutionary Biology, 13, 336-347.

VIGNIERI, S. N., LARSON, J. G. \& HOEKSTRA, H. E. 2010. The selective advantage of crypsis in mice. Evolution, 64, 2153-2158.

VOGEL, S. 1994. Life in Moving Fluids: The Physical Biology of Flow.

VOLBERG, G., WUTZ, A. \& GREENLEE, M. W. 2013. Top-Down Control in Contour Grouping. PLoS ONE, 8, 1-14.

VONDERHEYDT, R., PETERHANS, E. \& BAUMGARTNER, G. 1984. Illusory contours and cortical neuron responses. Science, 224, 1260-1262.

WAGEMANS, J., ELDER, J. H., KUBOVY, M., PALMER, S. E., PETERSON, M. A., SINGH, M. \& VON DER HEYDT, R. 2012a. A Century of Gestalt Psychology in Visual Perception: I. Perceptual Grouping and Figure-Ground Organization. Psychological Bulletin, 138, 1172-1217. 
WAGEMANS, J., FELDMAN, J., GEPSHTEIN, S., KIMCHI, R., POMERANTZ, J. R., VAN DER HELM, P. A. \& VAN LEEUWEN, C. 2012b. A Century of Gestalt Psychology in Visual Perception: II. Conceptual and Theoretical Foundations. Psychological Bulletin, 138, 1218-1252.

WALLACE, A. R. 1889. Darwinism: An exposition of the theory of of natural selection with some of its applications. London, Macmillian.

WANG, S., GE, F. \& LIU, T. 2006. Evaluating edge detection through boundary detection. EURASIP J. Appl. Signal Process., 2006, 213-213.

WEBSTER, R. J., CALLAHAN, A., GODIN, J. G. J. \& SHERRATT, T. N. 2009. Behaviourally mediated crypsis in two nocturnal moths with contrasting appearance. Philosophical Transactions of the Royal Society B-Biological Sciences, 364, 503-510.

WERTHEIMER, M. 1923. Studies concerning the Theory of Shape. Psychologische Forschung, 4, 301-350.

WIDDER, E. A. 2010. Bioluminescence in the Ocean: Origins of Biological, Chemical, and Ecological Diversity. Science, 328, 704-708.

WILKINSON, D. M. \& SHERRATT, T. N. 2008. The art of concealment. The Biologist, $55,10-15$.

WILSON, D., HEINSOHN, R. \& ENDLER, J. A. 2007. The adaptive significance of ontogenetic colour change in a tropical python. Biology Letters, 3, 40-43.

WILSON, R. A. \& KEIL, F. C. 2001. The MIT encyclopedia of the cognitive sciences, Cambridge, Massachusetts, MIT Press.

WOLFE, J. M. 1998. Visual search. University College 
WYZISK, K. \& NEUMEYER, C. 2007. Perception of illusory surfaces and contours in goldfish. Visual Neuroscience, 24, 291-298.

YAO, J. G., GAO, X., YAN, H. M. \& LI, C. Y. 2011. Field of Attention for Instantaneous Object Recognition. PLoS ONE, 6, 1-8.

YORZINSKI, J. L., PATRICELLI, G. L., BABCOCK, J. S., PEARSON, J. M. \& PLATT, M. L. 2013. Through their eyes: selective attention in peahens during courtship. The Journal of Experimental Biology, 216, 3035-3046.

YOSHIDA, A., MOTOYAMA, M., KOSAKU, A. \& MIYAMOTO, K. 1997. Antireflective nanoprotuberance array in the transparent wing of a hawkmoth,Cephanodoeshylas. Zool. Sci., 14, 737-741.

ZANFORLIN, M. 1981. Visual perception of complex forms (anomalous surfaces) in chicks. Italian Journal of Psychology, 8, 1-16.

ZAR, J. H. 1999. Biostatistical Analysis, London, Prentice Hall.

ZYLINSKI, S., DARMAILLACQ, A. S. \& SHASHAR, N. 2012. Visual interpolation for contour completion by the European cuttlefish (Sepia officinalis) and its use in dynamic camouflage. Proceedings of the Royal Society B-Biological Sciences, $279,2386-2390$.

ZYLINSKI, S. \& JOHNSEN, S. 2011. Mesopelagic Cephalopods Switch between Transparency and Pigmentation to Optimize Camouflage in the Deep. Current biology : $C B, 21,1937-1941$.

ZYLINSKI, S., OSORIO, D. \& SHOHET, A. J. 2009. Perception of edges and visual texture in the camouflage of the common cuttlefish, Sepia officinalis. 
Philosophical Transactions of the Royal Society B-Biological Sciences, 364, 439448. 


\section{APPENDICES}

\section{Appendix A.}

\section{Table A.1: Literature summary for background matching}

\begin{tabular}{|c|c|c|c|}
\hline Species & $\begin{array}{c}\text { Laboratory } \\
\text { (L), Field (F), } \\
\text { Human } \\
\text { Predator (H) } \\
\text { or } \\
\text { Observation } \\
\text { (Ob) }\end{array}$ & Description of evidence & Reference \\
\hline \multicolumn{4}{|l|}{ Colour matching } \\
\hline Catocala moths & $\mathrm{L}$ & $\begin{array}{l}\text { Moths' colour affects survivorship on tree } \\
\text { species of distinct colors }\end{array}$ & $\begin{array}{l}\text { Pietrewicz \& Kamil } \\
\text { (1977) }\end{array}$ \\
\hline Moths & $\mathrm{F}$ & $\begin{array}{l}\text { Moths' coloration match their backgrounds' } \\
\text { colour differently throughout the season. } \\
\text { Moths, on average, better match background } \\
\text { colour for the temporal period when they are } \\
\text { present. }\end{array}$ & Endler (1984) \\
\hline $\begin{array}{l}\text { Limpets, Pollicipes } \\
\text { polymerus \& } \\
\text { Semibalanus cariosus }\end{array}$ & $\mathrm{F}$ & $\begin{array}{l}\text { (a) two species choose different inter-tidal } \\
\text { zone habitat, which matches their colors (b) } \\
\text { when translocated to contrasting color, both } \\
\text { species' survival decreases }\end{array}$ & Mercurio et al. (1985) \\
\hline $\begin{array}{l}\text { Spring peeper, Hyla } \\
\text { crucifer }\end{array}$ & $\mathrm{L}$ & $\begin{array}{l}\text { (a) Frogs switch between dark and light } \\
\text { colorations depending on their substrate (b) } \\
\text { on a striped background they match the } \\
\text { localised color, and when straddling between } \\
\text { two lines, they select an intermediate colour. }\end{array}$ & Kats \& Dragt (1986) \\
\hline $\begin{array}{l}\text { Stonefly nymph, } \\
\text { Paragnetina media }\end{array}$ & $\mathrm{FL}$ & $\begin{array}{l}\text { Showed a preference for substrate that } \\
\text { matched its own color. Further, this choice } \\
\text { persisted on highly visually heterogeneous } \\
\text { environment }\end{array}$ & $\begin{array}{l}\text { Feltmate \& Willians } \\
\text { (1989) }\end{array}$ \\
\hline $\begin{array}{l}\text { Poplar hawkmoth, } \\
\text { Laothoe populi }\end{array}$ & $\mathrm{F}$ & $\begin{array}{l}\text { (a) final instar caterpillar changes colour to } \\
\text { match its substrate (b) yellow-green morph } \\
\text { had higher survivorship on substrates that it } \\
\text { matches, such a difference was not present } \\
\text { for the white morph }\end{array}$ & $\begin{array}{l}\text { Edmunds \& Grayson } \\
1990\end{array}$ \\
\hline $\begin{array}{l}\text { Pelagic shrimp, } \\
\text { Sargassum natans }\end{array}$ & $\mathrm{L}$ & $\begin{array}{l}\text { (a)Colour resembles background colour of leaf } \\
\text { fronds. (b) some species show substrate } \\
\text { selectivity that enhances colour matching }\end{array}$ & Hacker \& Madin (1991 \\
\hline
\end{tabular}


Primitive stick insect,

Timema cristinae

Marine snail, Litorina

saxatilis

Salamander,

Ambystoma barbouri

Pygmy grasshopper, Tetrix undulata

Tropical python, Morelia virdis

Benthic fish, Lucania

spp.

Plasticine mice

Threespine

stickleback,

Gasterosteus

aculeatus

Grasshopper, Acrida

ungarica

Pinnipeds(seal and sea lions)

Lagomorphs
Colour morphs show differential predation when switching between preferred food plants that their colors closely represent

Colour morph that matches substrate are more concealed

(a) Larvae change colour to match background environment (b) some species show habitat selection based on colour matching

Differential habitat selection and consequential survival difference Pythons switch from leaf litter to canopy as they develop and this is paralleled with an ontogenetic colour change from yellow or red juveniles to green adults. This colour change minimises visual disparity between the python and its background color, as perceived by avian predators.

(a) Fish change brightness to match that of their backgrounds

Mouse light and dark morphs survive best in sand dunes of equivalent background colors.

This was tested using plasticine mouse models Colour plasticity differs between sympatric benthis and limnetic species, with the benthic species showing greater plasticity also having a visual background with more heterogeneity

There is a higher proportion of brown morph grasshoppers as the proportion of brown grass increases

(a) Arctic species that are vulnerable to terrestrial predators are more likely to be white, when controlling for phylogeny (b) Deep water off-shelf species are more likely to be dark in color, when controlling for phylogeny

Dark coloration is associated with woodland habitat, but not when controlling for phylogeny (b) Pale coloration associated with desert, tundra and barren land habitats, a trend that is strengthened when controlling for phylogeny (c) gray coloration associated with rocky habitats, but not when controlling for phylogeny.
Sandoval (1994)

Johannesson \&

Ekendahl (2002)

Garcia \& Sih (2003)

Ahnesjo \& Forsman

Wilson et al. (2007)

Cox et al. (2009)

Vignieri et al. (2010)

Clarke \& Schluter

(2011)

Pellissier et al. (2011)

Caro et al. (2012)

Stoner et al. (2003)

\section{Contrast matching}

Cuttlefish, Sepia

officinalis (a) in high contrast visual environment the patch size (texon) determines different characteristic camouflage patterns (b) when patch size is constant cuttlefish fine tune their contrast
Barbosa et al. (2008)
L

g


Cuttlefish, Sepia

officinalis

Feather lice,

Phthiraptera
$\mathrm{Ob}$
Lowering luminance changes coloration from mottles to more contrasting 'disruptive'

Ecoparasites match luminosity of multiple avian host species. Interesting evidence of cryptic coloration out of the context of predator-prey interactions.
Chiao et al. (2010)

Bush et al. (2010)

\section{Surface texture}

matching

Guppy, Poecilia
reticulata
Cuttlefish, Sepia
officinalis
Blackbird spp.,
Icteridae

Bat, Chiroptera

Ob

Grasshopper, Acrida ungarica

Seal and sea lions, Pinnipeds

$\mathrm{F}$
L In the presence of predators, populations evolve more spots and they change their size and length

$\mathrm{L}$

The uniform body pattern can change the size of the stipple pattern to match background at an accurate spatial scale

(a) Egg patterning is highly variable, with texture quantified using wavelet analysis (b) the degree of each species' egg pattern disparity matches the substrates' disparity.

Bats that don't live in colonies are more likely to have pelage markings. This textural presence could have camouflage function, as natural selection would favor added protection in small colony living species, where the dilution effect of group living is diminished The proportion of grasshoppers with striped patterns vary according to habitat composition

$\mathrm{Ob} \quad$ Species in well-lit shallow water are more likely to have spotted appearance, when controlling for phylogeny
Endler 1980, 1982, 1989

Zylinski et al. (2009)

Westmoreland (1996)

Santana et al. (2011)

Pellissier et al. (2011)

Caro et al. (2012)

\section{Direction aligning}

Cuttlefish, Sepia officinalis

Geometric moths

F L

$\mathrm{F}$

Moths, Catocala spp and Larentiinae

representatives

\section{Response to match horizontal and vertical} aspects of visual background matching Moths mediate their orientation to match their patterns' orientations with that of their backgrounds' directionality

$\mathrm{F} \mathrm{L} \quad$ Moths mediate their orientation to enhance their survivorship, on a background with directionality

\section{Melanism colour matching}

Peppered moth, Biston

betularia

Dark melanistic morph has higher survivorship during industrial era (when trees are soot coated) compared to pale morph
Barbosa et al 2008

Kang et al. (2012)

Webster et al. 2009 
Peppered moth, Biston

betularia

Pygmy grasshoppers, Tetrix subulata
Pale morph has higher survivorship in post industrial ('soot-less')forest, compared to melanistic morph
(a) In a post fire environment the melanistic form was harder to detect in the heterogeneous background, especially in dark patches and (b) multiple populations showed shifts of common colour morph to melanistic form

\begin{tabular}{llll}
\hline Luminance matching & & & \\
\hline $\begin{array}{l}\text { Cuttlefish, Sepia } \\
\text { officinalis }\end{array}$ & L F & $\begin{array}{l}\text { Lowering luminance changes coloration from } \\
\text { mottles to more contrasting 'disruptive' }\end{array}$ & Chiao et al. 2010 \\
\hline Scale matching & & Medium sized patches mediate change in & Chiao et al 2010 \\
\hline $\begin{array}{l}\text { Cuttlefish, Sepia } \\
\text { officinalis }\end{array}$ & LF & contrast &
\end{tabular}

Optimally resembling a background --Non-random matching of common background elements

\begin{tabular}{|c|c|c|c|}
\hline Artificial moths & $\mathrm{F}$ & $\begin{array}{l}\text { (a) The more common an element is in a } \\
\text { background (i.e. grey stones), the higher } \\
\text { survival accrued from background matching }\end{array}$ & Cooper \& Allen (1994) \\
\hline Artificial moths & $\mathrm{L}$ & $\begin{array}{l}\text { Random samples of a background differ in } \\
\text { their survivorship. }\end{array}$ & Merilaita \& Lind (2004) \\
\hline Artificial moths & $\mathrm{L}$ & $\begin{array}{l}\text { Achromatic background matching yields } \\
\text { longer search time than chromatic matching }\end{array}$ & Stobbe et al. (2009) \\
\hline Artificial moths & $\mathrm{L}$ & $\begin{array}{l}\text { Spatially irregular arrangements of } \\
\text { background elements increase search time, in } \\
\text { both simple and complex visual backgrounds }\end{array}$ & $\begin{array}{l}\text { Dimitrova \& Merilaita } \\
\text { (2011) }\end{array}$ \\
\hline \multicolumn{4}{|l|}{$\begin{array}{l}\text { Size dependent } \\
\text { background matching }\end{array}$} \\
\hline $\begin{array}{l}\text { Marine spider crabs, } \\
\text { Herbstia condyliata }\end{array}$ & $\mathrm{F} \mathrm{Ob}$ & $\begin{array}{l}\text { Size dependent evolution of camouflage } \\
\text { decoration between species of majoid crabs }\end{array}$ & $\begin{array}{l}\text { Hultgren \& Stanowicz } \\
(2009)\end{array}$ \\
\hline Caterpillars & $\mathrm{L}$ & $\begin{array}{l}\text { Background matching at the shape level } \\
\text { through masquerade is dependent on size } \\
\text { matching }\end{array}$ & $\begin{array}{l}\text { Skelhorn et al. (2010); } \\
\text { Skelhorn \& Ruxton } \\
\text { (2013) }\end{array}$ \\
\hline $\begin{array}{l}\text { Marine kelp crab, } \\
\text { Pugettia producta }\end{array}$ & $\mathrm{F}$ & Size-specific habitat selection of kelp crabs & $\begin{array}{l}\text { Hultgren \& Stachowicz } \\
(2010)\end{array}$ \\
\hline $\begin{array}{l}\text { Cuttlefish, Sepia } \\
\text { officinalis }\end{array}$ & $\mathrm{L}$ & $\begin{array}{l}\text { As the checker size of background changes, } \\
\text { the relative size of cuttlefish expressed } \\
\text { coloration changes. This is indirect evidence } \\
\text { that there is size dependent background } \\
\text { matching. }\end{array}$ & $\begin{array}{l}\text { Chiao et al. (2007); } \\
\text { Barbosa et al. (2008); } \\
\text { Chiao et al. (2009) }\end{array}$ \\
\hline
\end{tabular}




\section{Appendix B.}

Table B.1: Literature summary for disruptive coloration

\begin{tabular}{ccc}
\hline Species & $\begin{array}{c}\text { Laboratory(L), } \\
\text { Field (F), }\end{array}$ & Description of evidence \\
Human Predator & \\
(H) or & \\
Observation (Ob) &
\end{tabular}

\begin{tabular}{|c|c|c|c|}
\hline Edge markings & & & \\
\hline $\begin{array}{l}\text { Isopod, Idotea } \\
\text { baltica }\end{array}$ & $\mathrm{F}$ & $\begin{array}{l}\text { (a) Occurrence of patches intersecting its } \\
\text { edge occurs more often than if randomly } \\
\text { sampling background (b) patches are high } \\
\text { contrast }\end{array}$ & Merilaita (1998) \\
\hline Artificial moths & $\mathrm{F}$ & $\begin{array}{l}\text { (a) Edge patches enhance survivorship (b) } \\
\text { differences persist when controlling for } \\
\text { density of patches }\end{array}$ & Cuthill et al. (2005) \\
\hline Artificial moths & $\mathrm{F}$ & $\begin{array}{l}\text { (a) Edge patches enhance survivorship (b) } \\
\text { asymmetry enhances survivorship, to a } \\
\text { lesser degree than edge patches }\end{array}$ & Cuthill et al. (2006) \\
\hline Artificial moths & $\mathrm{F}$ & $\begin{array}{l}\text { Edge-intersecting patches treatments } \\
\text { survive longer than background matchers }\end{array}$ & $\begin{array}{l}\text { Schaefer \& Stobbe } \\
(2006)\end{array}$ \\
\hline Artificial moths & $\mathrm{H}$ & $\begin{array}{l}\text { (a) Edge patches enhance survivorship and } \\
\text { have a longer search time (b) treatments } \\
\text { with edge patches and no background } \\
\text { matching survived poorly, suggesting that } \\
\text { for disruption to provide a camouflaged } \\
\text { benefit, background matching properties } \\
\text { are also required }\end{array}$ & Fraser et al. (2007) \\
\hline Artificial moths & $\mathrm{F}$ & $\begin{array}{l}\text { Edge-intersecting patches treatments have } \\
\text { less detectable lined boundaries, assessed } \\
\text { by computer vision algorithms }\end{array}$ & $\begin{array}{l}\text { Stevens \& Cuthill } \\
(2010)\end{array}$ \\
\hline
\end{tabular}

Coincidental disruption

$\begin{array}{llll} & \text { Where wing-body-wing colour patterns } & \text { Cuthihll \& Szekely } \\ \text { Artificial moths } & \text { F } & \text { align there is highest survivorship } & (2009)\end{array}$

Contrasting patterned marking

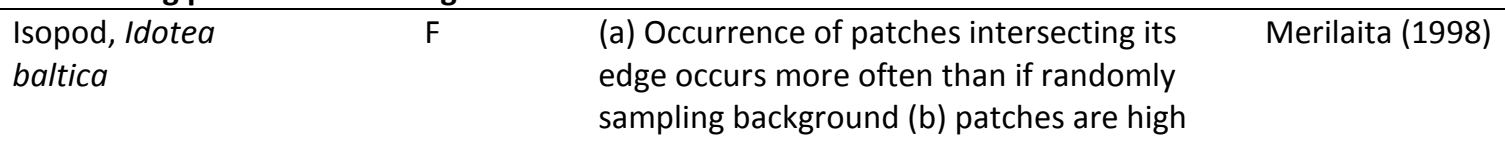


contrast

Artificial moths

Cuttlefish, Sepia

officinalis

Artificial moths

Artificial moths
Edge intersecting patches increase survival. This result only holds for non-random background matching. For optimal background matching, disruption has an equal level of concealment

(a) Contrast of coloration exceeds that of background (b) high contrast markings are displayed in certain checkerboard backgrounds (large light objects on overall dark backgrounds, whose texons are roughly the size of the animal's white square component; increasing substrate intensity; and increasing high-spatialfrequencies) (c ) High contrast and mottled coloration types are often mixed, suggesting a combination of disruption and background matching

No evidence that contrast that exceeds that of background benefits survival. Methods note: high contrast was achieved by setting one of the bi-colours of the target to a luminance higher than found in background. Therefore a large amount of area was changed color, instead of a small localised change in contrast at patches margins
Merilaita \& Lind (2004)

Hanlon et al. (2009), Chiao et al. (2005), Kelman et al. (2007), Chiao et al. (2007)

Stevens et al. (2006)

No effect, but limited within treatment

Dimitrova \& Merilaita replication

\section{Bisecting stripes}

Butterfly, Anartia

$\mathrm{F}$ Removal of white bisecting wing stripe had

fatima no effect on survivorship

\section{Disruption provide background independent camouflage}

Artificial moths
$\mathrm{F}$

$\mathrm{H}$

Edge-intersecting patches treatment with no background matching survives longer than cryptic background matcher Edge-intersecting patches improve concealment at the expense of background similarity Silberglied et al. (1980)

Schaefer \& Stobbe

(2006) (2009) 


\section{Appendix C}

Table C.1: GLMs of arcsin transformed mean proportion missed (survivorship) for each moth species, with three main effects (Relative moth orientation, Tree rotation (both fixed effects) and Tree (random effects)) and all pairwise interactions. Test statistics reported for the GLM are: $\mathrm{F}_{\mathrm{S}}[\mathrm{dfs}]$, sig. $(* * *=\mathrm{p}<0.001, * *=\mathrm{p}<0.005, *=\mathrm{p}<0.05$, $\mathrm{p}>0.05=\mathrm{NS})$.

\begin{tabular}{lll}
\hline GLMs factors and interactions & C. cerogama & E. intermediata \\
\hline Relative moth orientation & $18.58[7,56],{ }^{* *}$ & $3.40[7,56]{ }^{* *}$ \\
Tree rotation & $11.66[1,8]{ }^{*}$ & $5.64[1,8]{ }^{*}$ \\
Tree & $11.32[8,2.69]^{* * *}$ & $27.22[8,8.78]^{* * *}$ \\
Relative moth orientation ${ }^{*}$ Tree rotation & $2.08[7,56] \mathrm{NS}$ & $0.96[7,56] \mathrm{NS}$ \\
Relative moth orientation ${ }^{*}$ Tree & $1.73[56,56]^{*}$ & $1.40[56,56] \mathrm{NS}$ \\
Tree*Tree rotation & $1.39[8,56] \mathrm{NS}$ & $7.84[8,56]{ }^{* * *}$
\end{tabular}



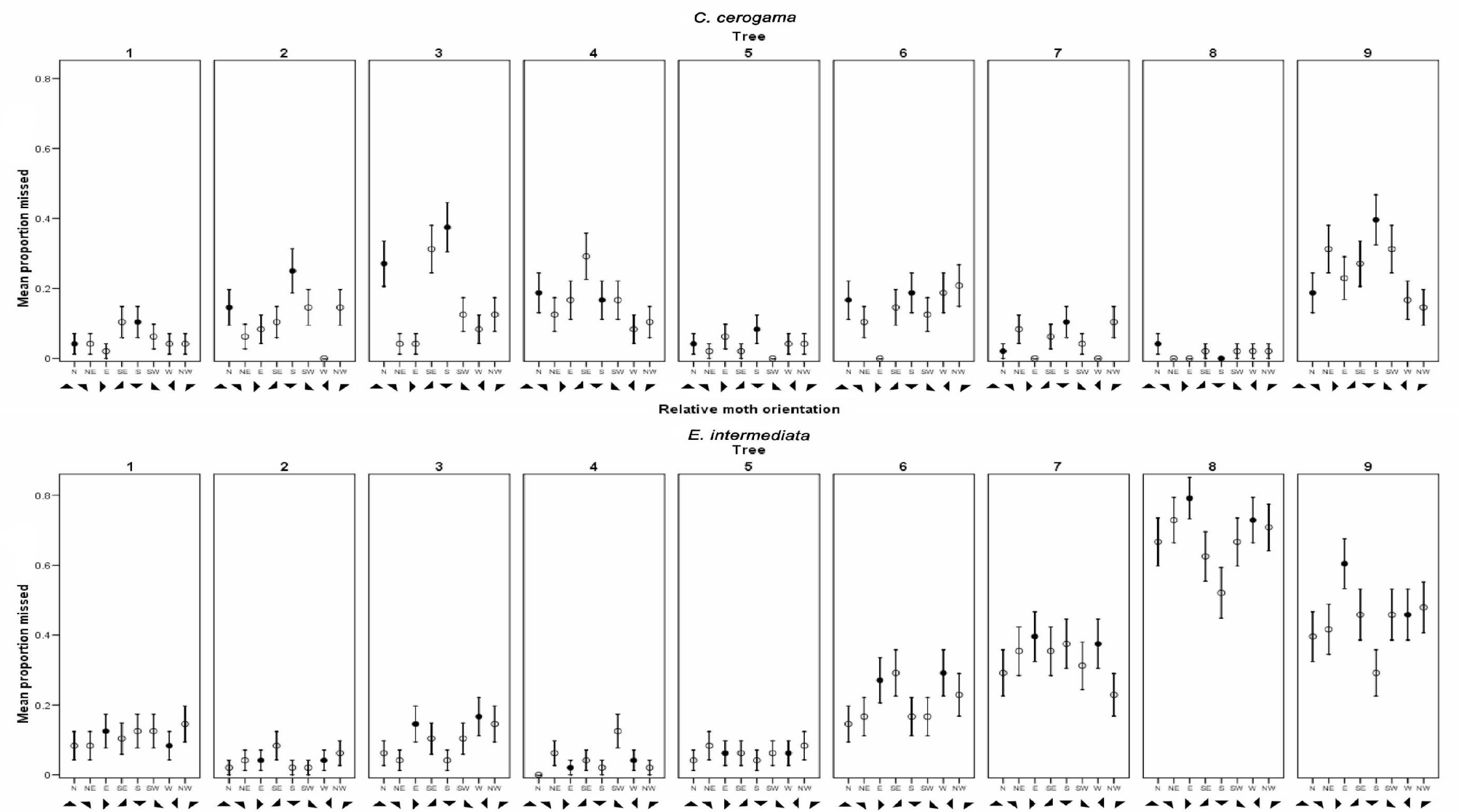

Relative moth orientation

Figure C.1a \& $\boldsymbol{b}$ : The mean ( \pm 1 standard error) moth targets missed per tree, for each tree image and relative orientation of $C$.

cerogama and E. intermediata (data on horizontal and vertical tree rotations combined). Differences in survivorship between moths with different orientations are more evident in those trees in which moths are relatively hard to detect. 

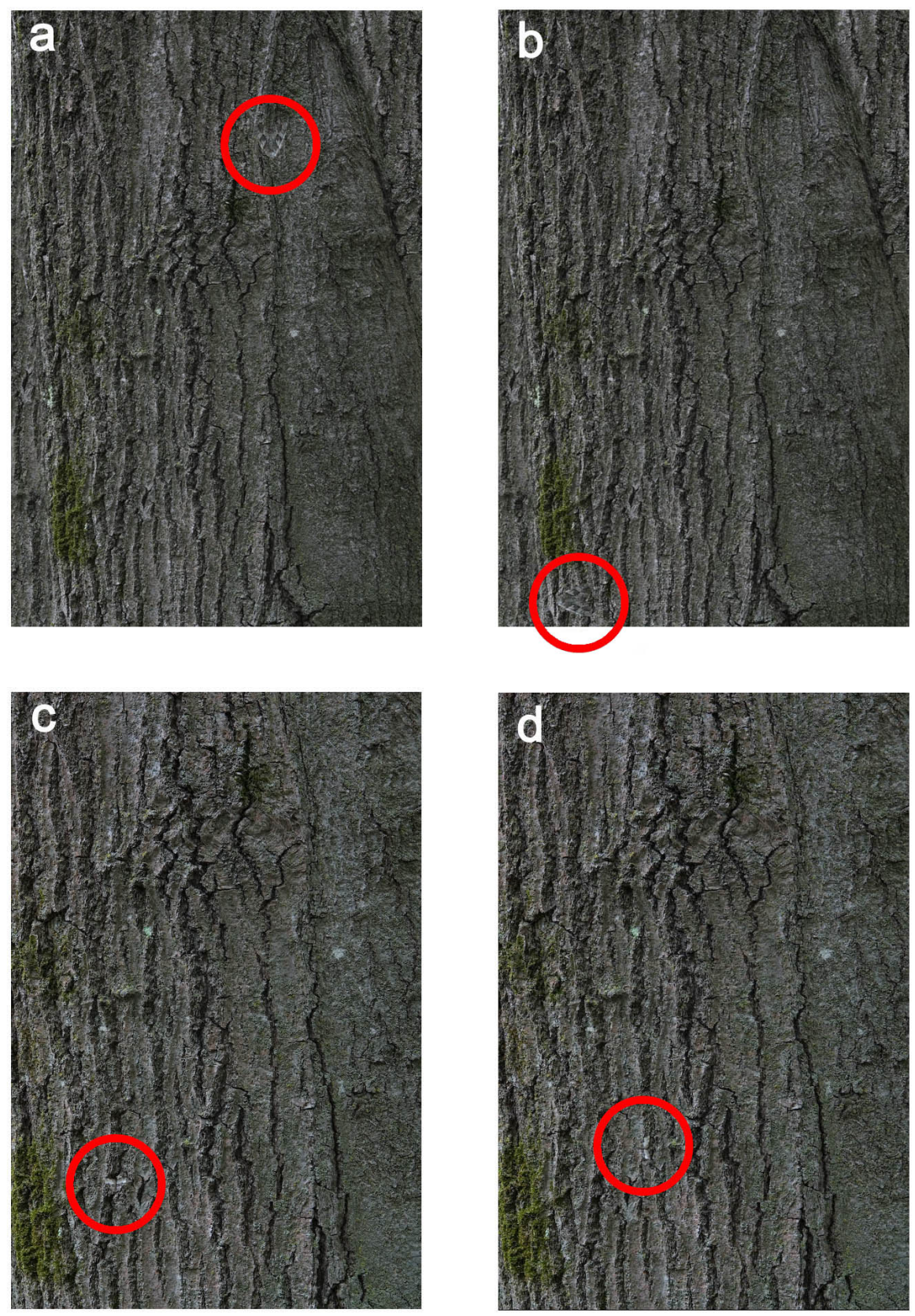

Figure C.2 a, b, c \& d: Colour images of C. cerogama (a and b) and E. intermediata (c and d) presented in their South (a and c) and West (b and d) relative orientations. These target moths are ringed in red. 


\section{Appendix D}

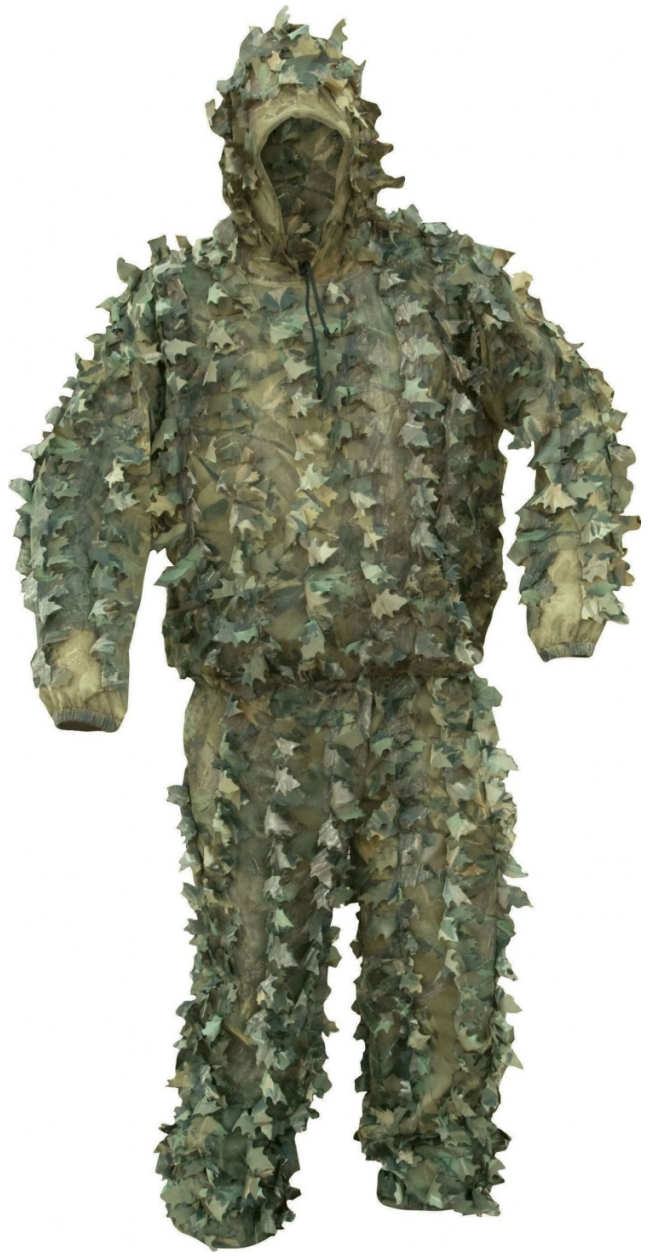

Figure D.1: A example of a ghillie suit used by humans for military and hunting purposes for concealment. It is speculated that by ruffling the outline of the wear results attains heightened camouflage. Ghillie suits are often worn by humans requiring a high level of camouflage when position in one location for a long time, such as military snipers and hunters. Image copyright of Jack Pyke of England ltd., of there LLCS Ghillie suit: http://www.jackpyke.co.uk/search/llcs-ghillie-suit.aspx 


\section{Fundamental frequencies \\ Unfiltered \\ Low-pass filtered}

(a)

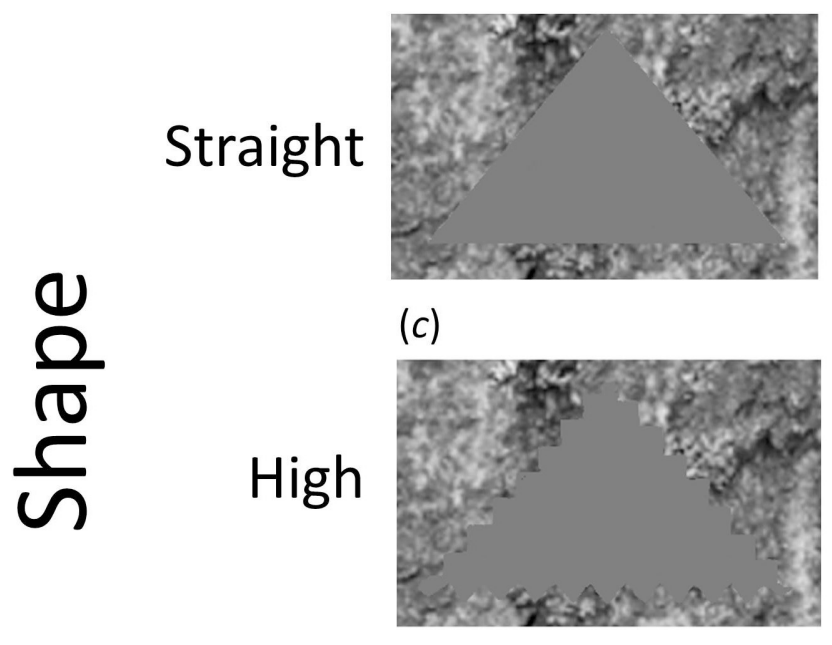

(e)

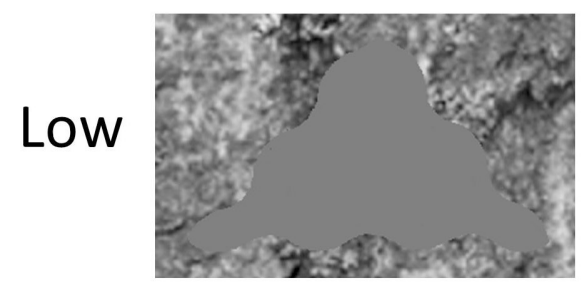

(b)

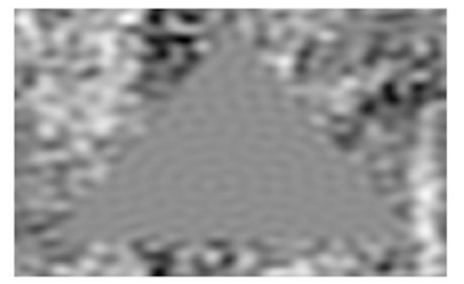

(d)

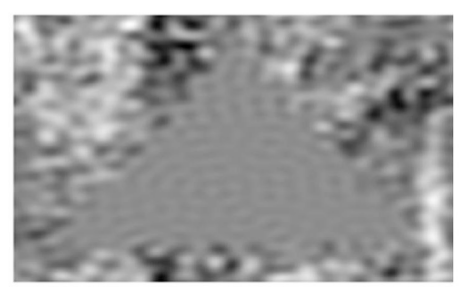

(f)

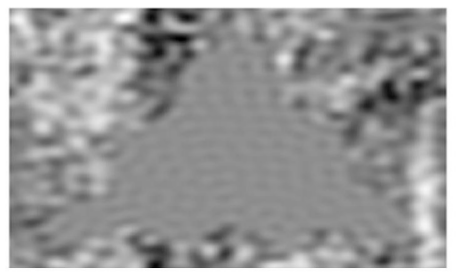

Figure D.2: Examples of how stimuli appear in presenting in my experiment, paired with a low-pass filtered. The different shape treatments (Straight $(a), \operatorname{High}(\underline{c}) \& \operatorname{Low}(e))$ clearly have distinct global shape, but when viewing these treatments under a restricted set of low spatial frequencies, this distinction seems less appearance for shape treatments (Straight $(b)$, High $(\underline{d}) \& \operatorname{Low}(f))$. Note that is seem difficult to distinguish between Straight and High shape, whilst the Low shape's course representation in low spatial frequencies remains distinct. 


\section{Appendix E}

\section{E.1 Sub-Appendix: Targets and backgrounds}

Human subjects were instructed to visually 'hunt' for artificial moth targets presented on grayscale photographs of sugar maples (Acer saccharum), with subjects moving and clicking a computer mouse to 'attack' the prey. To derive the targets, digital photographs of sugar maple trunks were thresholded at their median grayscale value. Thresholded areas were then colored dark and light gray based on the original tree image's $25^{\text {th }}$ and $75^{\text {th }}$ grayscale percentiles. From these bi-coloured tree images, random $\mathrm{x}-\mathrm{y}$ coordinates were generated for each photo and a bicoloured triangle cropped from that location (thus simulating well-camouflaged moths on a tree trunk, figure $1 a$ ). The coloration properties of each potential target was measured using MatLab’s Image Processing Toolbox (MathWorks, Natick, MA, USA). Targets varied naturally in the overall proportion of dark area ('proportion dark'), as well as the distribution of dark and light patches (Appendix Fig. E.2). To minimise collinearity between target characteristics, I selected 63 targets non-randomly from a pool of targets to achieve a diverse combination of coloration properties (by dividing the range of values of proportion dark and edge-intersecting patches into thirds, using a 3 x 3 sub-sampling matrix to select targets). As all targets were derived from backgrounds using the same procedure, they should have similar background matching properties sensu (Endler, 1984), although some will be closer to the mode than others. Pilot studies showed that the addition of $2 \%$ Gaussian noise to the bicoloured targets (using a filter applied in Adobe 
Photoshop to give the targets a subtle textured appearance) increased target concealment; this was particularly necessary given the size limit placed on the target because of the eye tracking methodology (the target size has a lower limit set by the measurement error of the eye tracker of $\left.2^{\circ}\right) . "$

The edge characteristics of each of the 63 moth-like targets were first categorised by the number of patches that intersected the target's triangular outline (Fig. E.2a). While this edge property is fixed for each target, the efficacy of such patterns in reducing detection will likely depend upon their interaction with their background. I therefore used a Canny edge detection algorithm (Chen He and Yung, 2008) to evaluate the extent to which the number of edge patches is likely a priori to influence edge detectability (Stevens and Cuthill, 2006). This algorithm enabled me to calculate the mean proportion of each of the 63 target's outline likely to be detectable in situ against 48 tree backgrounds and thereby involved the processing of a total of 3024 unique images. As predicted, there was a positive association between the number of edge-intersecting patches of a target and the mean proportion of the target's outline that was undetected in the algorithm $\left(F_{1,62}=21.1, p<0.001 ; \mathrm{R}^{2}=0.29 ;\right.$ Fig. E.3 $)$, indicating that the number of edge patches is a robust metric for the visibility of the target's outline.

It is worth noting that the current study's methodology differs somewhat from previous studies of disruptive coloration which compared a small range of distinct treatment types. A strength of this study is that 63 target types were included, allowing us 
to explore the effect of edge-intersecting patches as a continuous variable, whilst controlling for background matching covariates. Two background matching properties (i.e. uniformity and darkness) and one putative measure of 'disruption' (i.e. edgeintersecting patches) were also included in my analysis. By nesting these three target coloration properties within each targetID, my Type III sums-of-squares GLM approach allows me to partition the effect of edge coloration from the possible confounding effects of background matching. Instead of artificially binning targets into categories based simply on the presence and absence of edge intersecting patches, I set out to test for functional prediction of disruptive coloration by investigating the continuum of edgeintersecting patches over a full range.

\section{E.2 Sub-Appendix: Tracking of eye movements}

The FaceLab ${ }^{\mathrm{TM}}$ eye tracker (Seeing Machine, Canberra, Australia) used in the current study provided a non-invasive (e.g. no chin brace or head mount) means of tracking eye movement, using a 3D computer vision model that was fitted to each subject's face. An infrared LED light source, reflecting from the subject's eye, was used to triangulate the X-Y screen coordinates at which the subject's gaze was focused. The stereo cameras and infrared light source were mounted between the subject and the screen (Sharp, LC-50LE442U 50” Aquos, resolution: 1920x1080, diagonal screen size: 49.5 inches, and refresh rate: $60 \mathrm{~Hz}$ ), and obscured a small horizontal strip at the bottom of the monitor. To compensate for this, my Visual Basic program did not place targets behind this masked section of the screen. 
The eye tracker measures the subject's eye position whilst their foveal vision samples the scene. While object recognition can in theory be achieved without attentional processing (namely, peripheral vision) (Julesz, 1984, Treisman, 1988), measuring foveal vision to derive a metric of recognition is appropriate since camouflaged targets typically have long search times, which demand close attention to details and high visual acuity (Treisman and Gelade, 1980, Di Lollo et al., 2000, Rao and Ballard, 1999, Lee and Mumford, 2003). Foveal vision was classified as being on the target if the subject's gaze was within a $1.5^{\circ}$ zone-of-interest around the target (beyond $1.5^{\circ}$, there is a dramatic drop off in foveal vision acuity (Palmer, 1999); Fig. 6.2 \& Fig. E.1) and during periods of eye fixation (and excluding saccades). The target size of $120 \times 60$ pixels $\left(3.9^{\circ} \times 2.2^{\circ}\right)$ against a $1200 \times 1600$ pixels background $\left(39^{\circ} \times 22^{\circ}\right.$; Fig. 6.1$)$ and the subjects' viewing distance of $1.8 \mathrm{~m}$ struck a balance between being large enough to identify subjects gaze on the target, yet not too large to render the target overtly conspicuous. Further, the eye tracker recorded the eyes' location every $1 / 60$ th of a second; some portions of this time were spent saccading, and therefore represent periods where limited visual information was gained. To exclude saccades from our analyses we filtered the data using a velocity-based threshold in which any eye movement with a velocity of greater than $20^{\circ}$ visual angles per second was identified as a saccade. This rule of thumb is a commonly used method to identify and exclude saccades that is based on the underlying physiology of the eyes.

The eye tracking machine's visual angle error ranged from $0.5-2.5^{\circ}$. Tracker calibration was carried out for up to 40 minutes for each subject prior to testing. Subjects 
whose visual angle error exceeded $2^{\circ}$ after calibration were excluded from participating in the experiment, leaving 48 subjects for testing. It was important in the study that the visual-hunting task represented an ecologically relevant level of difficulty for hunting camouflaged objects. Indeed this task seems to have been challenging for the subjects, as up to $18 \%$ of some moth target types presented were not discovered at all, despite close attention. 


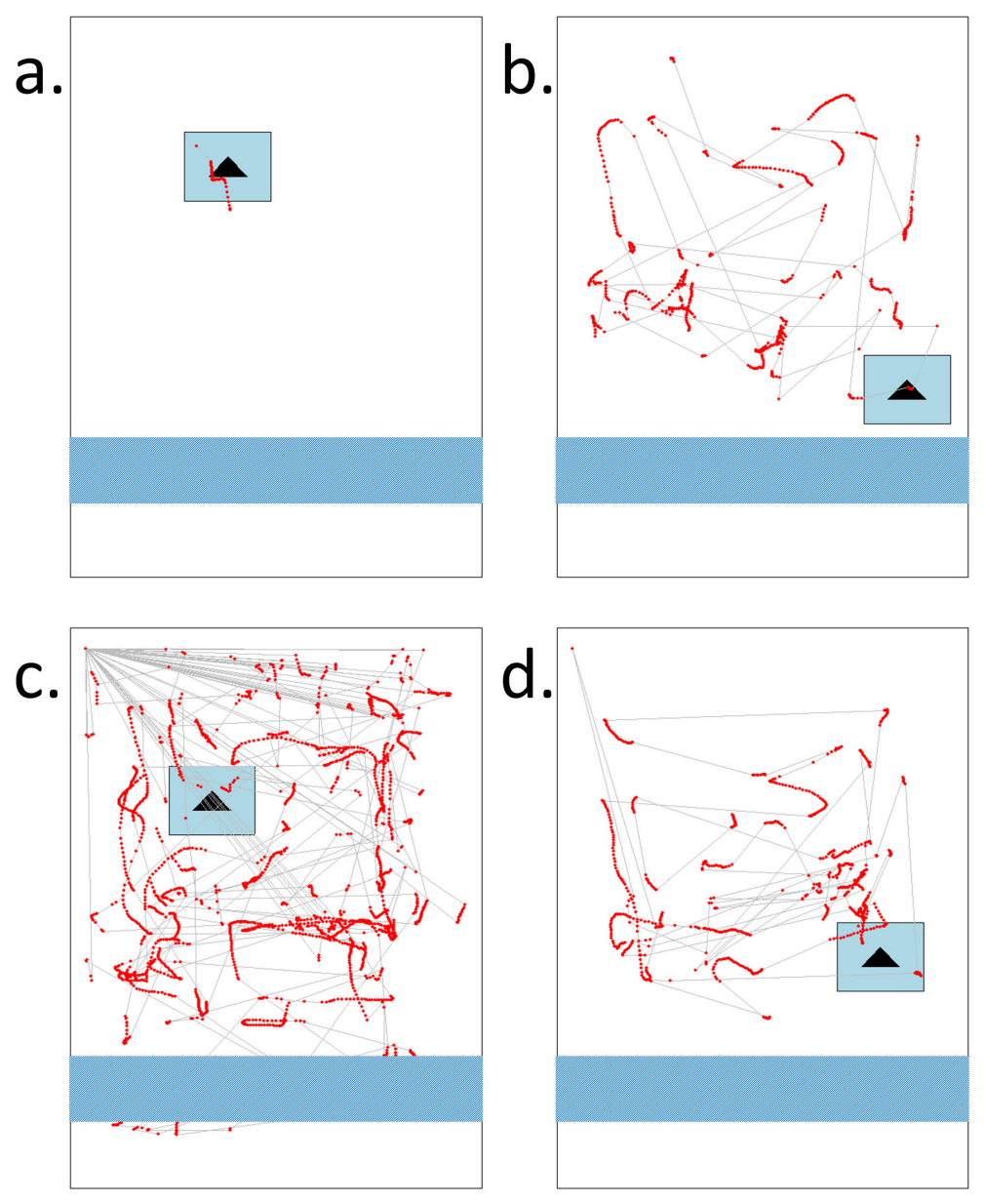

Figure E.1: Examples of visual search paths selected to display a range of subject search times and inspection times recorded by the FaceLab ${ }^{\mathrm{TM}}$ eye tracker. The blue rectangle represents that portion of the screen effectively masked out from the experiment, due to eye tracking cameras obscuring the screen (Fig. 6.1). The red dots represent where subject's gaze intersects with the screen (with one sample recorded every $17 \mathrm{~ms}$ ). This zone has an additional $1.5^{\circ}$ visual angle of padding on each side (horizontal and vertical) from the target, a distance determined by the foveal vision's acuity (Palmer, 1999). (a) low search time, low inspection time; (b) high search time, low inspection time; (c) high search time, high inspection time; (d) low search time, high inspection time. 

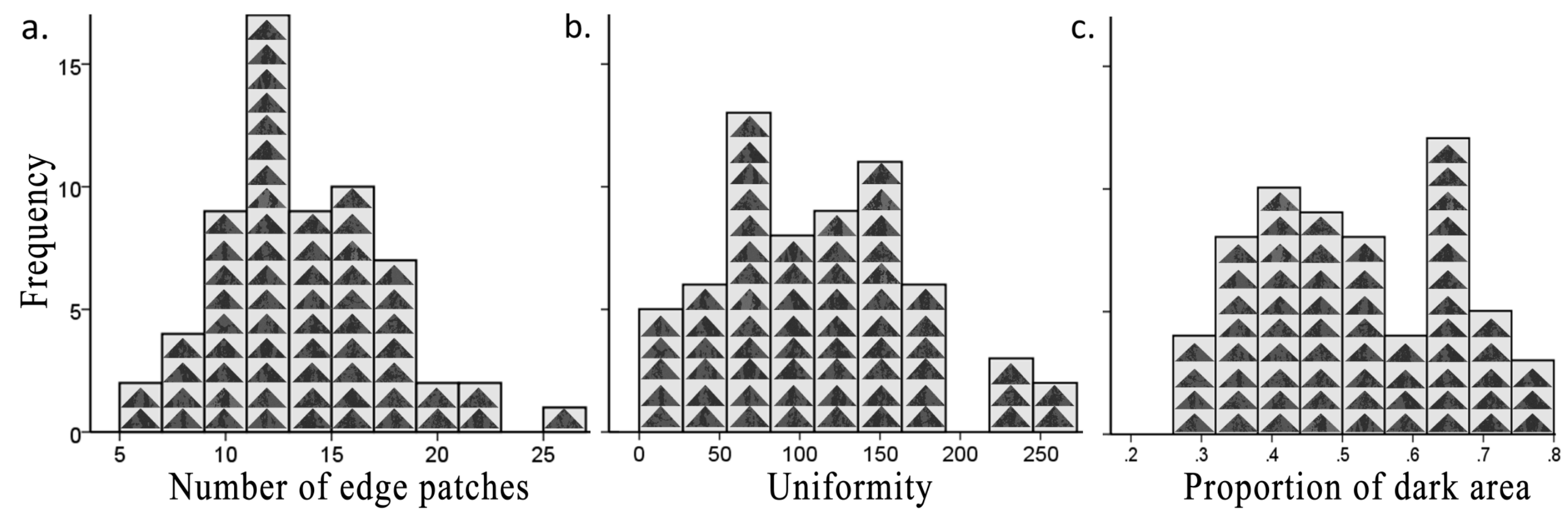

Figure E.2: Frequency distributions of the 63 moth targets depicting variation in $(a)$ the number of edge patches, $(b)$ uniformity, and $(c)$ proportion of surface area covered by dark patches. This high resolution figure also serves as a gallery of all targets; zoom in to view the coloration properties of individual targets. See Fig. E.8 for scatter graphs showing how these three colour pattern characteristics are correlated. 


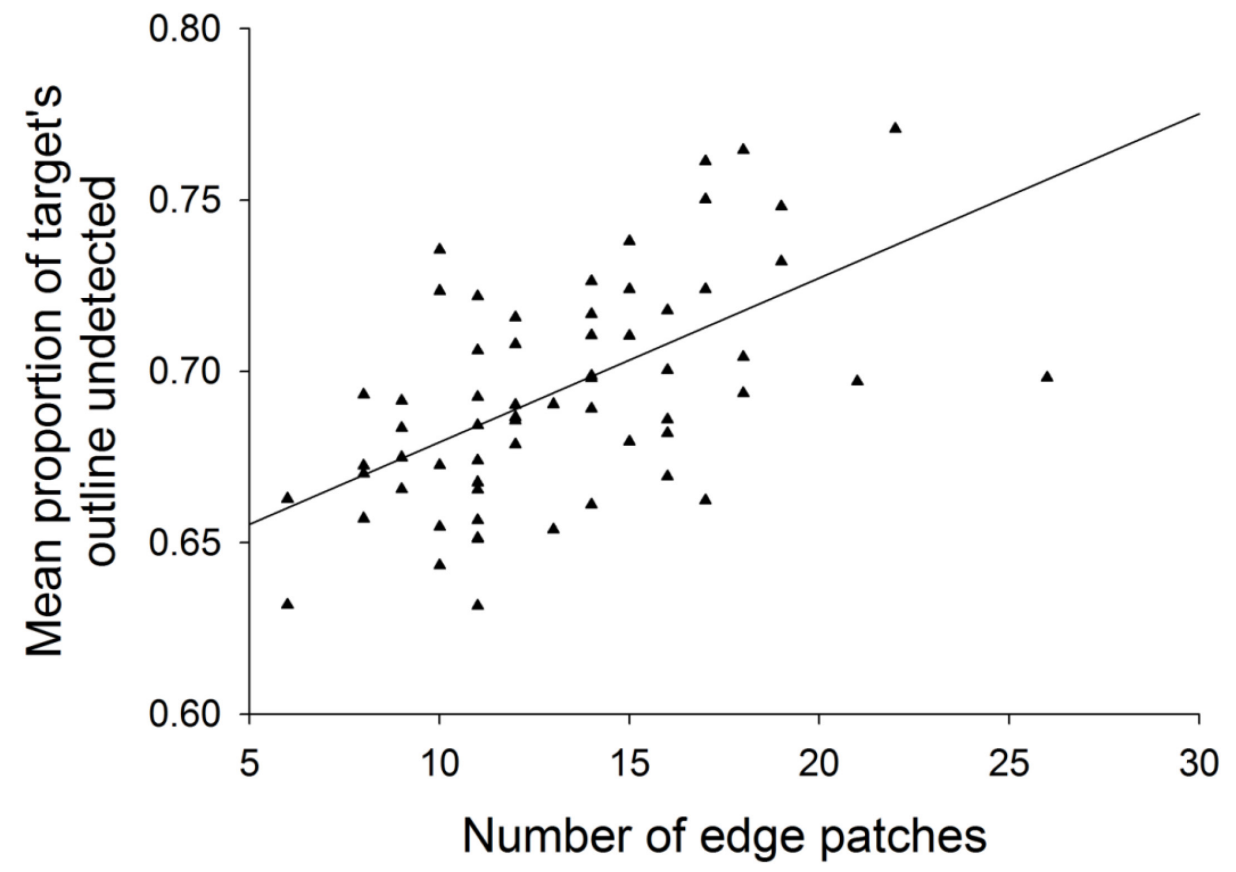

Figure E.3: Edge-intersecting patches break up a target's outline. A target's number of edge-intersecting patches is positively correlated with the target's mean proportion of undetected outline (as estimated by a Canny edge detection algorithm (Chen He and Yung, 2008), with high and low thresholds of 0 and 0.35 , and $\sigma=1.4$ ). As the number of edge-intersecting patches increases, the Canny algorithm fails to detect more of the target's outline (when presented in situ against tree backgrounds). Line represents the fitted ordinary least-squares regression. 


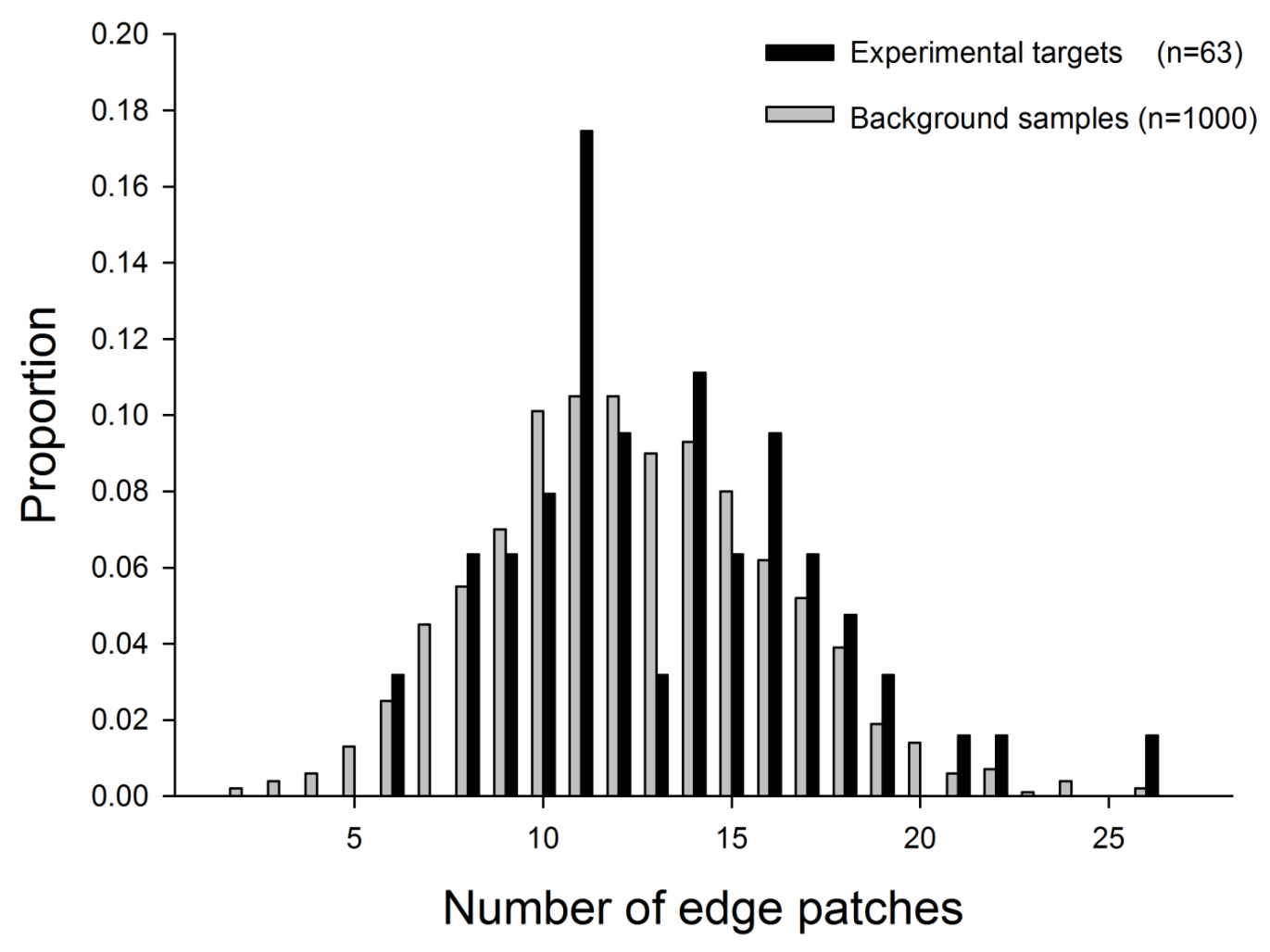

Figure E.4: Distribution of edge-intersecting patches for backgrounds and experimental target types. Triangular targets were randomly sampled 100 times each from the 10 experimental trees $(n=1000)$. By repeatedly measuring the frequency of edgeintersecting patches on these triangle outlines, I calculate that the median number of edge-intersecting patches is 12 . The numbers of edge-intersecting patches on the moth targets (derived from different trees) span a range of values representative of the backgrounds on which they are present. However, those targets with more or less than 12 edge patches could be considered less representative of the background in terms of the average number of edge patches than a randomly placed triangle would be expected to contain. 


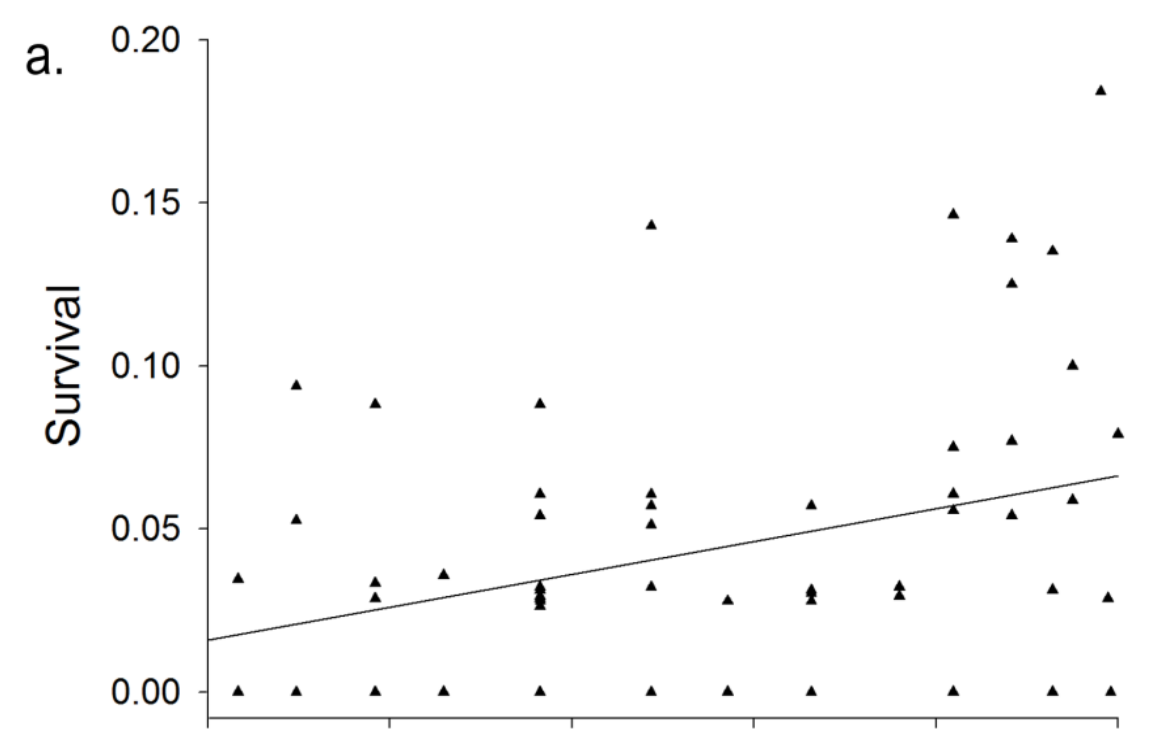

b.

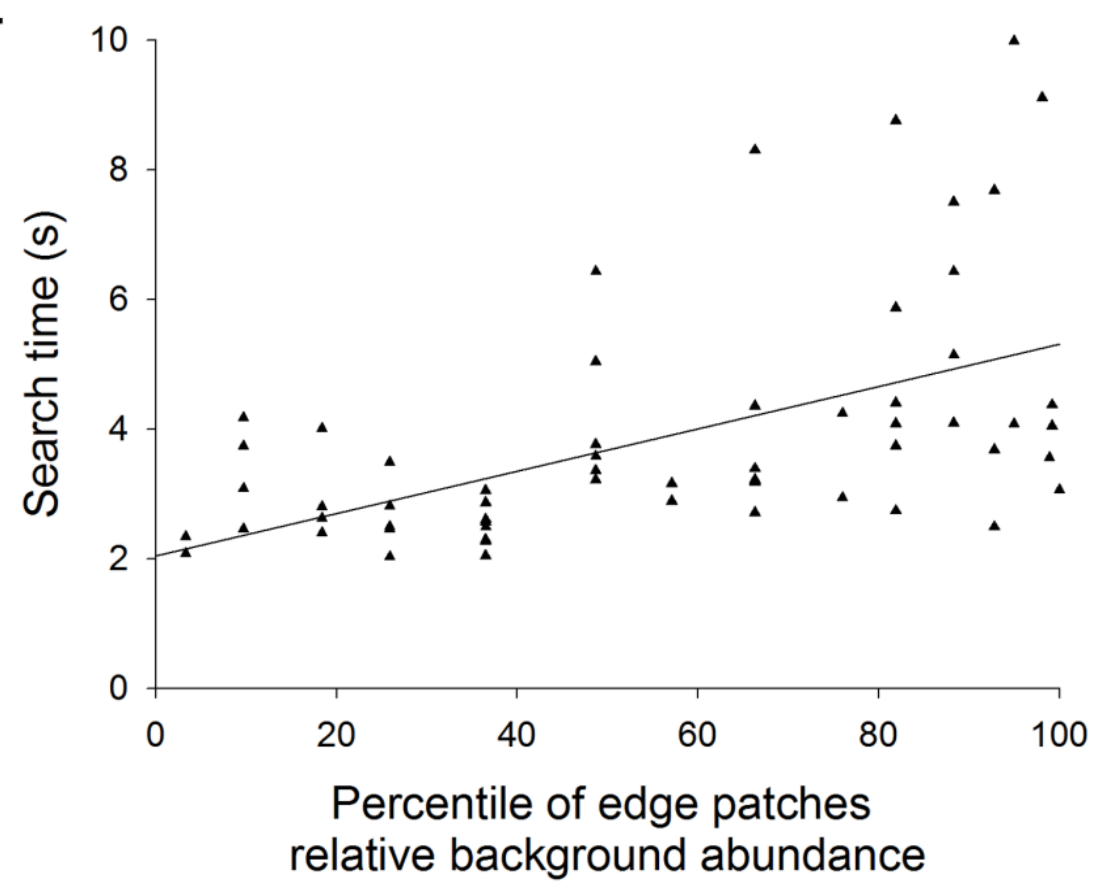

Figure E.5: Targets with high number of edge-intersecting patches do not match their environments but tend to go undetected. This figure is similar to Fig. 6.3 (in the main text), with the $\mathrm{x}$-axis variable transformed from absolute number of edge patches, to 
percentile of edge patches on the target relative to the background distribution (Fig. E.4). If there was no effect of edge-intersecting patches on survivorship, the line-of-best-fit would have a slope of zero. If an effect of edge-intersecting patch was driven by background matching, a curve would be fitted with a peak at the $50^{\text {th }}$ percentile (i.e. the most abundant background element). What I found is an effect of edge-intersecting patches on survival, with an increase in edge patches leading to a linear increase in target survivorship. Targets that are less representative of the background with more edgeintersecting patches have (a) heightened survival (proportion of each target that went undiscovered over all presentations; $\mathrm{F}_{1,59}=7.97, p=0.007$; Table E.1) and (b) longer search times $\left(\mathrm{F}_{1,59}=13.55, p=0.001\right.$; Table E.1). A complementary approach that also suggests background matching does not explain edge patches affect on targets' detectability is that that there is non-significant effect of its quadratic term (see Table E.5). 


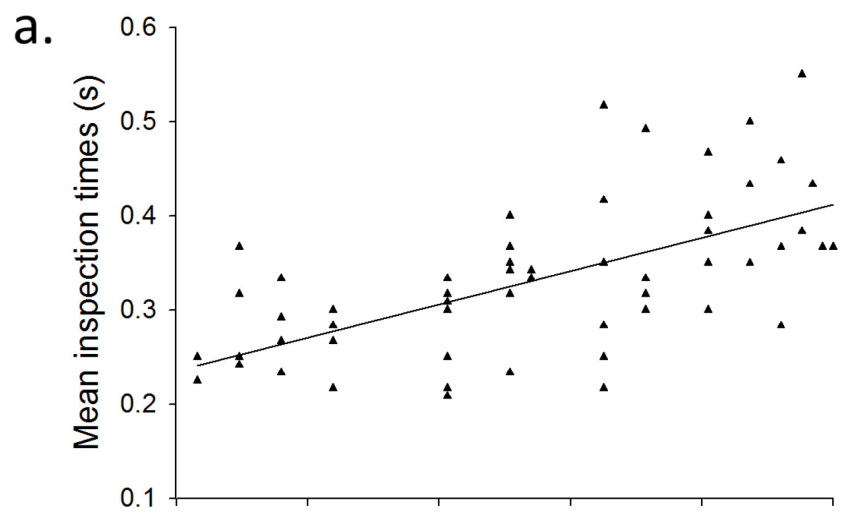

b.

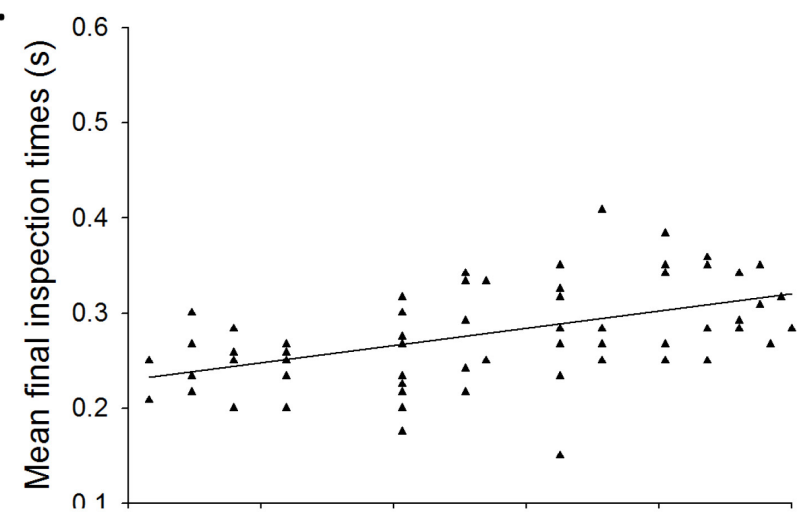

C.

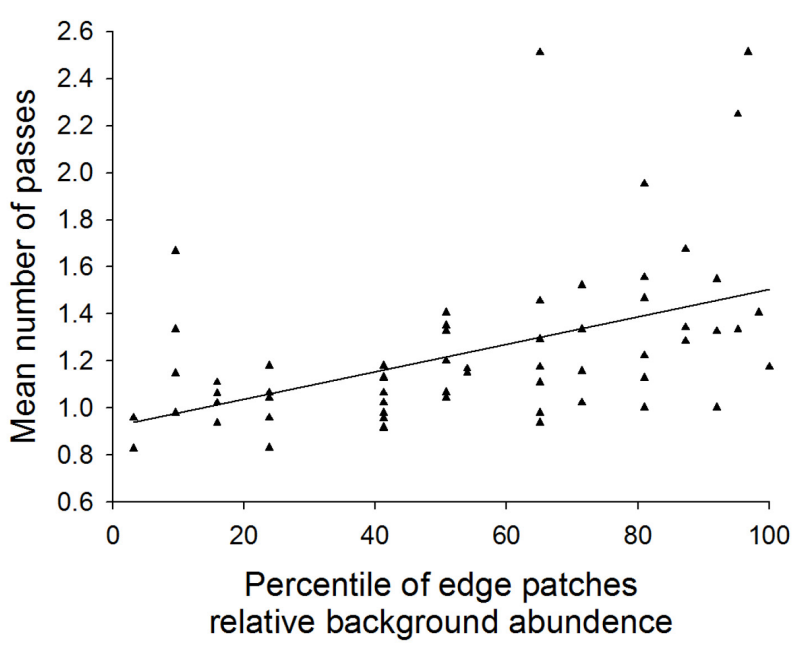

Figure E.6: Targets with high numbers of edge-intersecting patches do not match their environments, but take longer to recognise. This figure is similar to Fig. E.4 (in the main text), with the x-axis variable transformed from absolute number of edge patches to 
percentile of edge patches relative background abundance (Fig. 6.4). Departure from the $50^{\text {th }}$ percentile leads to targets edge properties becoming less common in the backgrounds, with targets to the left having fewer edge-intersecting patches and targets to the right having more edge-intersecting patches. Targets that are less representative of the background with more edge-intersecting patches take $(a)$ longer to be inspected $\left(\mathrm{F}_{1,59}=7.47, p=0.008\right.$; rounded to the nearest hundredth of a second; Table E.1) and (b) are more likely to be passed over $\left(\mathrm{F}_{1,59}=12.449, p=0.001\right.$; Table E.1). Variability in object recognition seems to be explained by a greater number of edge-intersecting patches, beyond that expected by background matching of common visual elements. I argue that these edge-intersecting patches have a disruptive function, as by break-up the visibility of targets outline (Fig E.5). 


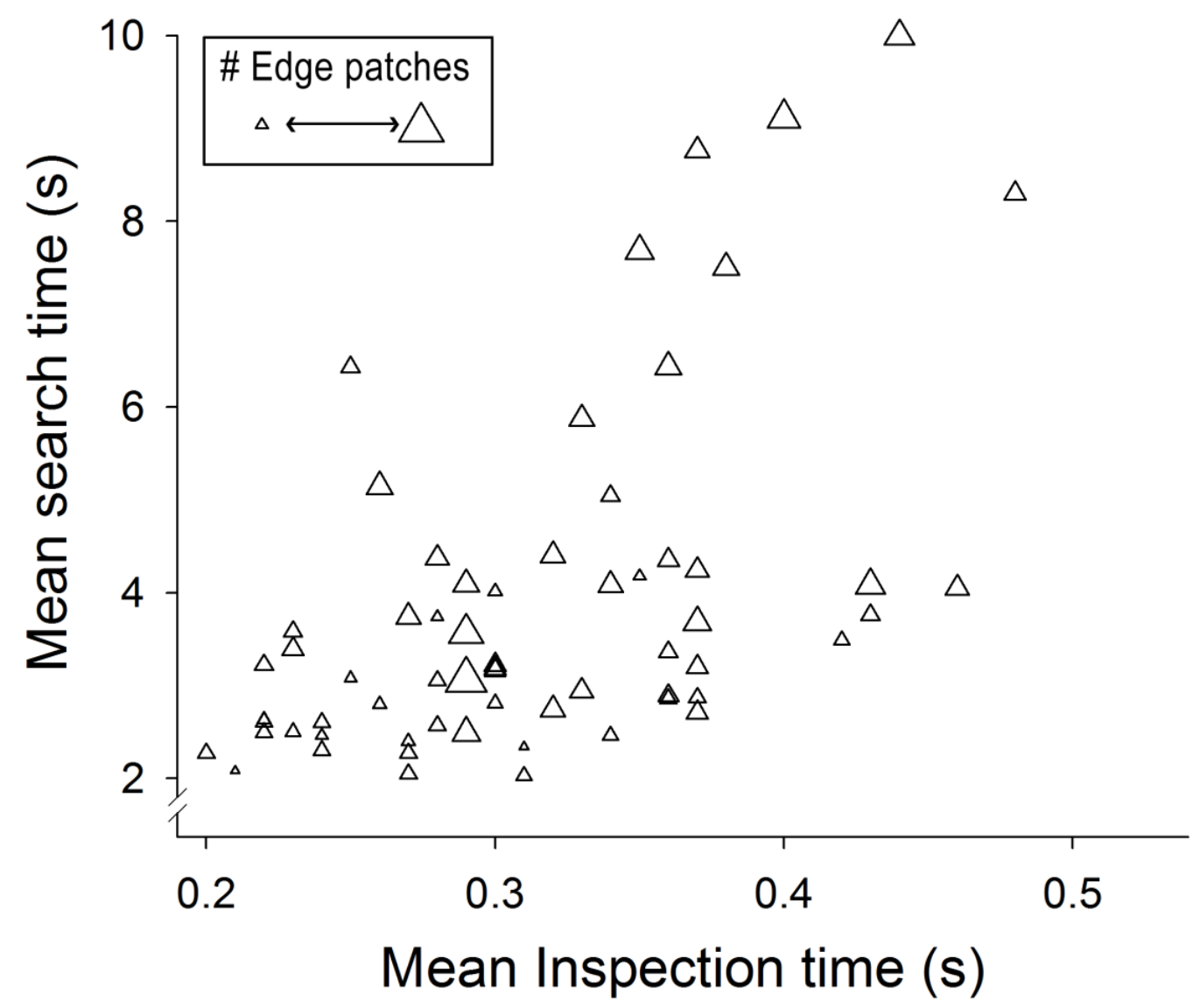

Figure E.7: Relationship between mean total inspection time and mean search time, mediated by disruption. Subjects spent increasingly longer periods visually inspecting detected moth targets with increasing time spent visually searching for them. This relationship appears to be mediated by number of edge patches on the targets, as denoted by the relative size of the triangular data points. The figure legend gives an example of the two targets with low (7) and high (18) number of edge intersecting patches. 

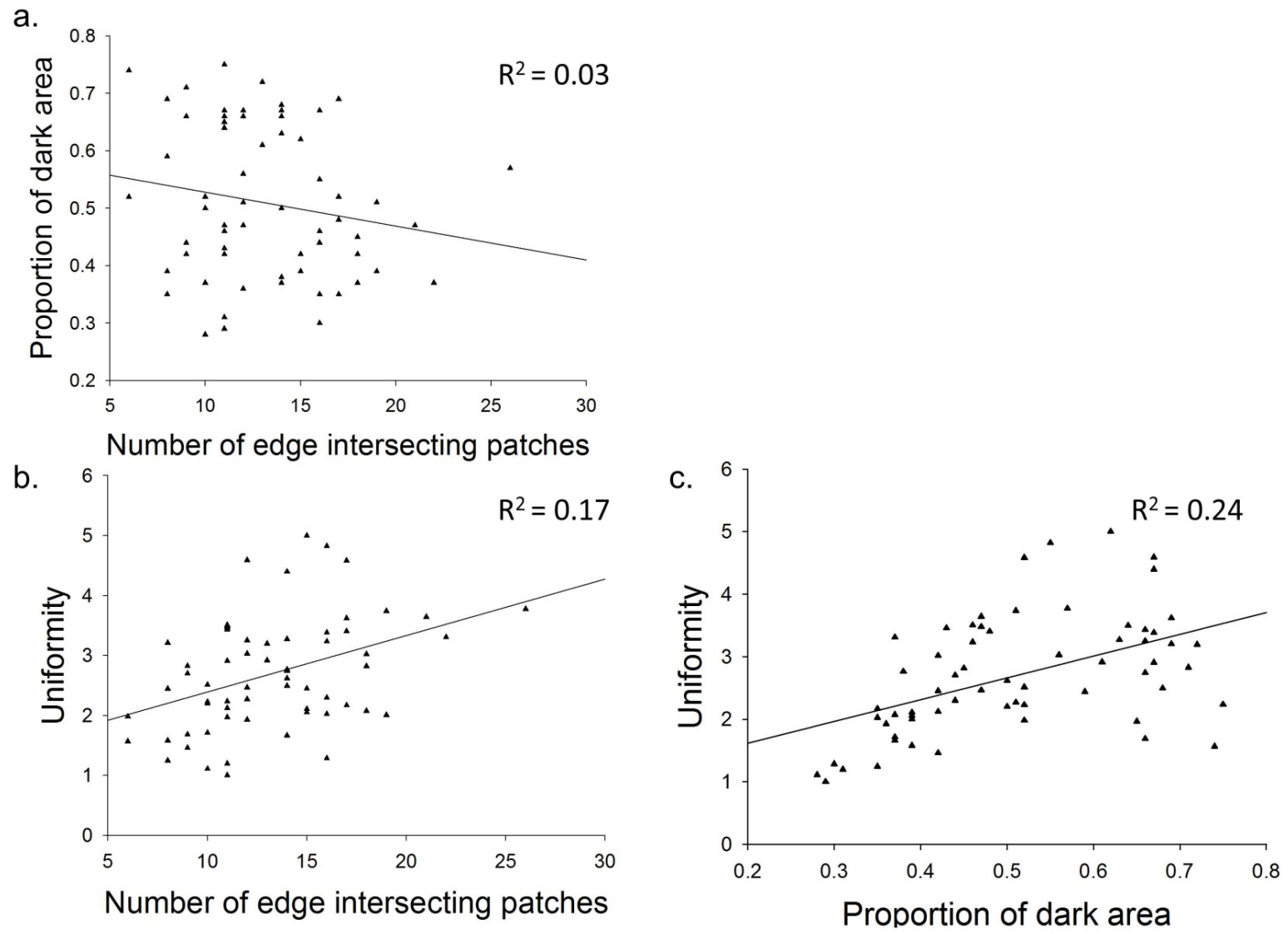

Figure E.8: Scatter plots showing the correlation between the three target coloration properties. ( $a$ ) edge-intersecting patches vs. proportion of dark area (b) edge-intersecting patches vs. uniformity and $(c)$ proportion of dark area vs. uniformity. All three combinations show a relatively low degree of correlation, with R-squared values all below 0.25 . 
Table E.1: Testing the effects of the moth targets' coloration properties on detection and recognition, when the edge patch abundance of each target was expressed as a percentile with respect to equivalent targets cut from the background. Each data column represents the fit of a separate general linear model, similar to Table 1 (main text), but with the number of edgeintersecting patches variable transformed to percentile of edge patches relative background abundance (see Fig. E.4-E.6). All fitted models included three target coloration properties as predictor variables (percentile of edge patches, total proportion of targets covered by dark area, and uniformity). These predictors were fitted using Type III sums of squares to control for the effect of correlated coloration properties. The geometric means for detectability and recognisability metrics were calculated across all 63 target presentations. Test statistics reported for the fitted models are $F$ values $(d f=1,59$ in all cases $)$

\begin{tabular}{|c|c|c|c|c|c|c|}
\hline \multicolumn{2}{|c|}{ Predictor variables } & \multicolumn{5}{|c|}{ Dependent variables } \\
\hline \multirow{2}{*}{$\begin{array}{l}\text { Type of } \\
\text { coloration }\end{array}$} & \multirow{2}{*}{$\begin{array}{c}\text { Target coloration } \\
\text { variable }\end{array}$} & \multicolumn{2}{|c|}{ Detectability } & \multicolumn{3}{|c|}{$\begin{array}{l}\text { Recognisability } \\
\text { Final inspection }\end{array}$} \\
\hline & & $\begin{array}{c}\text { Survival } \\
\text { (log transform) }\end{array}$ & $\begin{array}{c}\text { Search Time } \\
\text { (log transformed) }\end{array}$ & Inspection time & time & Number of $p$ \\
\hline Edge & $\begin{array}{l}\text { Percentile of edge } \\
\text { patches }\end{array}$ & $7.970 p=0.007 * *$ & $13.550, p=0.001 * * *$ & $22.77, p<0.001$ & $12.876, p<0.001$ & $6.943, p=0.01$ \\
\hline Overall & Total prop. of dark area & $2.010, p=0.163$ & $0.496, p=0.484$ & $0.157, p=0.693$ & $0.307, p=0.582$ & $0.005, p=0.94$ \\
\hline Overall & Uniformity & $0.027, p=0.870$ & $6.430, p=0.014 *$ & $0.975, p=0.327$ & $0.128, p=0.722$ & 4.359, $p=0.04$ \\
\hline
\end{tabular}


Table E.2: Results of logistic regression testing for the effects of the target coloration on survivorship. My original analyses used mean target survival and mean time to detection over all trials as the response variables (see Table 1 in the main paper), implicitly treating other factors that may contribute to intra-trial and inter-trial variability as noise. Here, I extend the analysis by controlling for the random effects of human subject, tree background and $\mathrm{X}-\mathrm{Y}$ coordinates of target placement on the monitor screen on target survivorship (see Table E.3 for the complementary analyses of detection and recognition time). A logistic regression was fitted in R using lmer from the lme4 library to model survivorship, with a Type I sums of squares. Human subject, tree background, X-Y screen placement coordinates, overall target coloration properties (uniformity, proportion of dark area) were included sequentially into the model before target edge coloration property (edge-intersecting patches). Each of these target colour pattern properties were nested within target type, such that the fitted model in R was:

$\operatorname{lmer}($ Survival $\sim(1 \mid$ Human $)+(1 \mid$ Tree $)+\mathrm{X}+\mathrm{Y}+(1 \mid$ TargetType $) /($ Uniformity + PropDarkArea + EdgePatches), family = binomial(link="logit")). The random factors of Human, Tree and TargetType are essentially blocks so their effects are not reported below. When analysing the full data set of thousands of trials, this nested structure allowed us to control for the 63 independent target types while investigating the effects of their coloration properties. My analyses indicated that the Y (but not X) screen location of the target affected its survival $(Z=-2.99 \mathrm{df}=1,2210, p<0.01$, see below). All target coloration properties (nested within target type) significantly contributed to survival (uniformity, $Z=-2.70$, df $=62,2210, p<0.01$; proportion of dark area, $Z=2.65, \mathrm{df}=62,2210, p<0.001$; edge- 
intersecting patches, $Z=-2.13, \mathrm{df}=62,2210, p<0.05)$. The number of edge-intersecting patches had qualitatively the same influence on survivorship as reported in the average data analysis. Interestingly, however, proportion of dark area was significant in the logistic regression model, which was not the case in the average data analyses; perhaps this is because the noise attributed to subject $\mathrm{Y}$, tree background, $\mathrm{X}-\mathrm{Y}$ screen placement are controlled for in the latter model, but also the higher degrees of freedom, generating a more powerful test. Statistical significance levels are denoted as $* p<0.05, * * p<0.01$, $* * * p<0.001$

\begin{tabular}{lcll}
\hline Logistic regression factors & $\begin{array}{l}\text { Parameter } \\
\text { estimate }\end{array}$ & $\boldsymbol{d f}$ & $\boldsymbol{p}$ \\
\hline $\mathrm{X}$ & $1.42 \times 10^{-4}$ & 1 & 0.635 \\
Y & $-8.22 \times 10^{-4}$ & 1 & 0.003 \\
Uniformity & $-4.84 \times 10^{-3}$ & 62 & 0.007 \\
Proportion of dark area & $3.44 \times 10^{-1}$ & 62 & 0.008 \\
Edge-intersecting patches & $-5.64 \times 10^{-2}$ & 62 & 0.033 \\
\hline
\end{tabular}


Table E.3: Fits of generalised linear mixed-effects models (GLMMs) to test for the effects of target coloration on detection and recognition. Here, I blocked for the random effects of human subject, tree background and X-Y coordinates of target placement on the monitor screen in a similar manner to that described in the fit of the logistic model (Table E.2 ) and analysed the four dependent variables separately. The error distribution of search time, inspection time and final inspection time were all assumed Gaussian, while the error distribution of the number of passes was assumed to be Poisson. For example, the fitted model in R's lmer package (Bates et al., 2011) for search time was: $\operatorname{lmer(Search~Time~}(1 \mid$ Human $)+(1 \mid$ Tree $)+\mathrm{X}+\mathrm{Y}+$

$(1 \mid$ TargetType $) /($ Uniformity + PropDarkArea + EdgePatches $)$, family = gaussian). Some transformations were performed to normalise the fitted models residuals. Once again, the results were qualitatively similar to the average data presented in the main paper (Table 6.1), with the p-values of edge-intersecting patches increasing in their significance. In these models, $\mathrm{p}$ values for number of passes with Poisson error were obtained using Markov Chain Monte-Carlo (MCMC) methods with 10,000 simulations using the language R (Baayen, 2011) package in $\mathrm{R}$. 
Table E.3 (conti.)

\begin{tabular}{|c|c|c|c|c|c|c|c|c|}
\hline \multirow[t]{5}{*}{ Predictor variables } & \multicolumn{8}{|c|}{ Dependent variables } \\
\hline & \multicolumn{2}{|c|}{ Detection } & \multicolumn{6}{|c|}{ Recognition } \\
\hline & \multirow{2}{*}{\multicolumn{2}{|c|}{$\begin{array}{l}\text { Search time } \\
\text { (log transformed) }\end{array}$}} & \multirow{2}{*}{\multicolumn{2}{|c|}{$\begin{array}{l}\text { Inspection time } \\
\text { (log transformed) }\end{array}$}} & \multirow{2}{*}{\multicolumn{2}{|c|}{$\begin{array}{l}\text { Final inspection time } \\
\text { (sqrt transformed) }\end{array}$}} & \multirow{2}{*}{\multicolumn{2}{|c|}{$\begin{array}{c}\text { Number of passes } \\
\text { (sqrt transformed) }\end{array}$}} \\
\hline & & & & & & & & \\
\hline & Estimate & $p$ & Estimate & $p$ & Estimate & $p$ & Estimate & $p \mathrm{MCMC}$ \\
\hline $\mathrm{X}$ & $1.47 \times 10^{-4}$ & $0.031 *$ & $1.00 \times 10^{-4}$ & 0.068 & $1.00 \times 10^{-4}$ & 0.209 & 0 & 0.929 \\
\hline $\mathrm{Y}$ & $6.28 \times 10^{-4}$ & $<0.001 * * *$ & $-2.00 \times 10^{-4}$ & $>0.001 * * *$ & $-8.00 \times 10^{-4}$ & $>0.001 * * *$ & $-4.0 \times 10^{-4}$ & $<0.001 * * *$ \\
\hline Uniformity & $2.07 \times 10^{-3}$ & $0.004^{* *}$ & $1.00 \times 10^{-3}$ & 0.051 & $-2.00 \times 10^{-4}$ & 0.669 & $1.70 \times 10^{-4}$ & $0.024 *$ \\
\hline Proportion of dark area & $-2.98 \times 10^{-2}$ & 0.446 & $6.29 \times 10^{-2}$ & 0.779 & $6.45 \times 10^{-2}$ & 0.777 & $-2.9 \times 10^{-2}$ & 0.930 \\
\hline Edge patches & $4.33 \times 10^{-2}$ & $<0.001 * * *$ & $2.87 \times 10^{-2}$ & $>0.001 * * *$ & $2.46 \times 10^{-2}$ & $0.002 * *$ & $2.99 \times 10^{-2}$ & $0.010 * *$ \\
\hline
\end{tabular}


Table E.4: Disruptive coloration mediates the contribution of recognition to target detectability. A general linear model (type III SS) was fitted to test the effects of both inspection time (total time focusing in vicinity of target) and target colour properties (number of edge-intersecting patches, proportion dark area and uniformity) on the mean total search time (i.e. the time taken to discover target). The total search time per target type was averaged across presentations for those presentations in which targets were discovered. The fitted model (in R) was: glm(SearchTimeAverage $\sim$ Uniformity + PropDarkArea + EdgePatches + InspectionTimeAverage + EdgePatches * Inspection TimeAverage, family = gaussian). As expected, a main effect of inspection time on mean search time was found, and further variance could be explained by an interaction term of inspection time*edge-intersecting patches. This interaction is clear in Fig E.9 where targets with low disruption (data points represented by the smaller triangles) have a shallow gradient between inspection time and search time; whilst targets with high disruption (data points represented by the larger triangles) have a steeper gradient. The significant interaction term might indicate that the contribution of recognition to total search time is mediated by disruption. Test statistics reported for the fitted models are $F$ values ( $\mathrm{df}=1,57$ in all cases).

\begin{tabular}{|c|c|}
\hline Predictor variables & $\begin{array}{c}\text { Dependent variable } \\
\text { Search time } \\
\text { (log transformed) }\end{array}$ \\
\hline Edge-intersecting patches & $8.89, p=0.004 * *$ \\
\hline Proportion of dark area & $1.32, p=0.26$ \\
\hline Uniformity & 6.37, $p=0.014 *$ \\
\hline Inspection time & $5.85, p=0.019 *$ \\
\hline Inspection time*Edge patches & $12.28, p=0.001 * * *$ \\
\hline
\end{tabular}


Table E.5: Testing the effects of the number of edge patches quadratic term on moth targets' detections. Each data column represents the fit of a separate general linear model. All fitted models included three target coloration properties as predictor variables (number of edge patches, total proportion of targets covered by dark area, and uniformity), with the addition of number of edge patches quadratic term. These predictors were fitted using Type I sums of squares, due to the quadratic term in the models. The geometric means for detectability metrics were calculated across all 63 target presentations. If edge patch effect on detectability was due to background matching, I would expect to see a significant effect of the quadratic term: as expected the quadratic term is not significant, suggested that another function, such as disruptive coloration, might account for edge patches concealment advantage. Test statistics reported for the fitted models are $F$ values ( $d f=1,58$ in all cases), slope coefficient and $p$ values.

\begin{tabular}{|c|c|c|c|}
\hline \multirow{2}{*}{$\begin{array}{l}\text { Type of } \\
\text { coloration }\end{array}$} & \multirow[t]{2}{*}{ Target coloration variable } & \multicolumn{2}{|c|}{ Detectability } \\
\hline & & $\begin{array}{l}\text { Survival (log } \\
\text { transform) }\end{array}$ & $\begin{array}{c}\text { Search Time (log } \\
\text { transformed) }\end{array}$ \\
\hline Overall & Total Prop. of dark area & $0.15,-0.010 p=0.706$ & $0.26,0.05, p=0.622$ \\
\hline Overall & Uniformity & $0.90,-0.352 p=0.348$ & $7.42,-0.118, p=0.009$ \\
\hline Edge & Number of edge patches & $12.37,0.047, p=0.001$ & $19.61,0.063, p<0.001$ \\
\hline Edge & $\begin{array}{l}\text { Number edge patches } \\
\text { quadratic }\end{array}$ & $0.10,-0.001, p=0.749$ & $2.66,-0.002, p=0.108$ \\
\hline
\end{tabular}

